\title{
Enantioselective N-Heterocyclic Carbene-Catalyzed Cascade Reaction for the Synthesis of Pyrroloquinolines via $\mathrm{N}-\mathrm{H}$ Functionalization of Indoles
}

\author{
Subrata Mukherjee, ${ }^{\dagger} \S$ Sayan Shee, $¥$ Thomas Poisson,,$\|, j$ Tatiana Besset $\|$ \\ and Akkattu T. Biju*, \\ $\dagger$ Organic Chemistry Division, CSIR-National Chemical Laboratory (CSIR-NCL), Dr. Homi \\ Bhabha Road, Pune - 411008, India

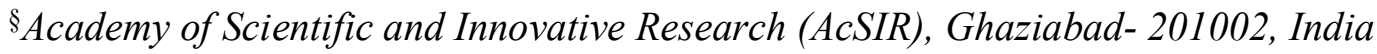 \\ $₫$ Department of Organic Chemistry, Indian Institute of Science, Bangalore-560012, India. \\ "Normandie Univ, INSA Rouen, UNIROUEN, CNRS, COBRA (UMR 6014), 76000 Rouen, \\ France \\ 'Institut Universitaire de France, 1 rue Descartes, 75231 Paris, France \\ atbiju@iisc.ac.in
}

\section{Supporting Information}

1. General Information $\quad$ S2

2. General Procedure for the Optimization of the Reaction Conditions $\quad$ S3

3. General Procedure for the Enantioselective Synthesis of Pyrroloquinolines S6

$\begin{array}{ll}\text { 4. X-ray data of } \mathbf{3 h} & \mathrm{S} 7\end{array}$

5 Synthesis and Characterization of Indole Derivatives $\quad$ S9

6. Synthesis and Characterization of Pyrroloquinoline Derivatives $\quad$ S12

$\begin{array}{ll}\text { 7. Functionalization of Pyrroloquinolines } & \text { S29 }\end{array}$

8. ${ }^{1} \mathrm{H}$ and ${ }^{13} \mathrm{C}$ NMR Spectra of Indole derivative S33

9. ${ }^{1} \mathrm{H}$ and ${ }^{13} \mathrm{C}$ NMR Spectra of Pyrroloquinoline Derivatives $\quad$ S38

10. HPLC data of Pyrroloquinoline Derivatives $\quad$ S65 


\section{General Information}

Unless otherwise specified, all reactions were carried out under an atmosphere of argon in flame-dried reaction vessels with Teflon screw caps. Dry DMSO was purchased from commercial sources and stored under argon over $4 \AA$ molecular sieves. The $\alpha, \beta$-unsaturated aldehydes 2a, 2e, 2f, 2m, 2t were purchased from commercial sources and were used without further purification, and others were synthesized following the literature procedure. ${ }^{1}$ Indole derivative 1d were synthesized by following the literature procedure. ${ }^{2}$ The triazolium salt $\mathbf{4}$ was synthesized following the literature procedure. ${ }^{3} 25{ }^{\circ} \mathrm{C}$ corresponds to the room temperature of the lab when the experiments were carried out.

Analytical thin layer chromatography was performed on TLC Silica gel 60 F 254 . Visualization was accomplished with short wave UV light or $\mathrm{KMnO}_{4}$ staining solutions followed by heating. Flash chromatography was performed on silica gel (230-400 mesh) by standard techniques eluting with Pet. Ether-EtOAc solvent system.

All compounds were fully characterized. ${ }^{1} \mathrm{H}$ and ${ }^{13} \mathrm{C}$ NMR spectra were recorded on Bruker Ultrashield spectrometer in $\mathrm{CDCl}_{3}$ or DMSO- $d_{6}$ as solvent. Chemical shifts $(\delta)$ are given in ppm. The residual solvent signals were used as references and the chemical shifts converted to the TMS scale $\left(\mathrm{CDCl}_{3}: \delta_{\mathrm{H}}=7.26 \mathrm{ppm}, \delta_{\mathrm{C}}=77.16 \mathrm{ppm}\right.$ and DMSO- $d_{6}: \delta_{\mathrm{H}}=2.50 \mathrm{ppm}, \delta_{\mathrm{C}}=$ $39.50 \mathrm{ppm})$. Infrared (FT-IR) spectra were recorded on a Perkin Elmer Spectrum BX spectrophotometer, $v$-max in $\mathrm{cm}^{-1}$. Optical rotations were measured on JASCO P-2000 polarimeter. HRMS (ESI) data were recorded on a Micromass Q-TOF Micro instrument. HPLC analysis was performed on Agilent Technologies 1260 Infinity II with UV detector.

\footnotetext{
${ }^{1}$ A. A. Wubea, A. Hüfner, C. Thomaschitz, M. Blunder, M. Kollroser, R. Bauer, F. Bucar, Bioorg. Med. Chem. 2011, 19, 567.

${ }^{2}$ Giardinetti, M.; Moreau, X.; Coeffard, V.; Greck, C. Adv. Synth. Catal. 2015, 357, 3501.

${ }^{3}$ J. R. Struble, and J. W. Bode, Org. Synth. 2010, 87, 362.
} 


\section{General Procedure for the Optimization of Reaction Conditions ${ }^{\mathrm{a}}$}

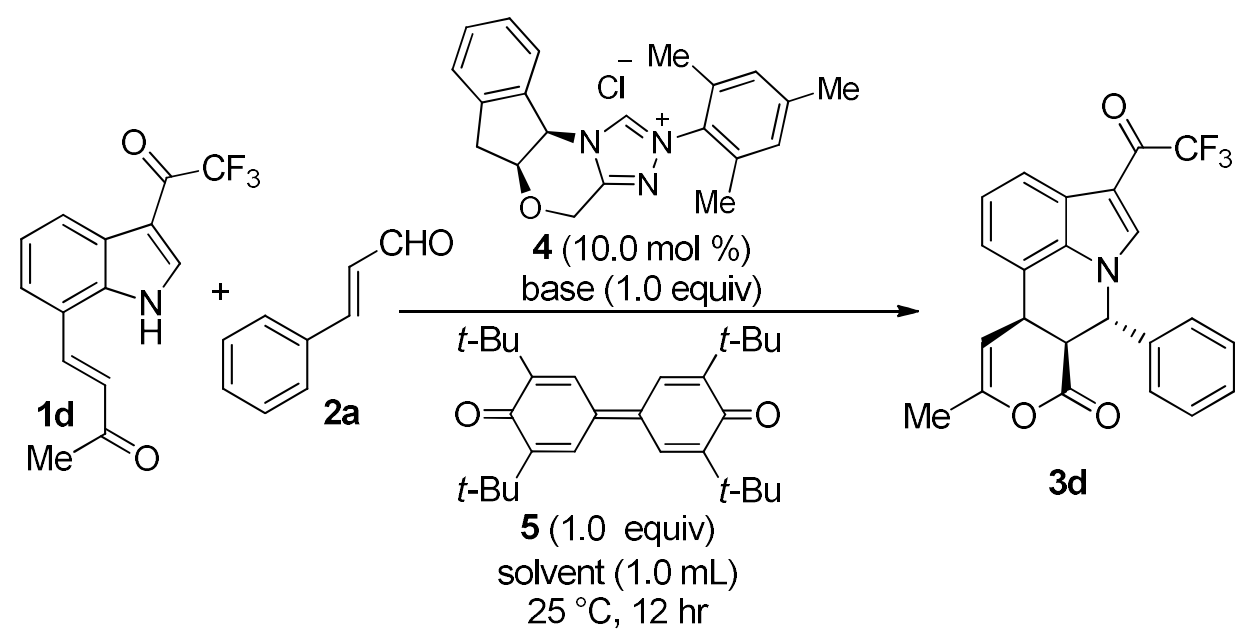

To a flame-dried screw-capped test tube equipped with a magnetic stir bar was added the triazolium salt $4(0.015 \mathrm{~g}, 0.0375 \mathrm{mmol})$ and $(E)-4$-(3-(2,2,2-trifluoroacetyl)-1H-indol-7-yl)but3-en-2-one 1d $(0.125 \mathrm{mmol})$, and enal $\mathbf{2 a}(0.125 \mathrm{mmol})$ was added. Then the screw-capped tube was evacuated and backfilled with argon. To this mixture was added solvent $(1.0 \mathrm{~mL})$ under argon atmosphere. The resultant reaction mixture was kept stirring at $25^{\circ} \mathrm{C}$. To this mixture base $(0.125 \mathrm{mmol})$ was successively added. After $12 \mathrm{~h}$, the reaction is quenched and the mixture was diluted with $\mathrm{CH}_{2} \mathrm{Cl}_{2}(2.0 \mathrm{~mL})$ and filtered through a short pad of silica gel and eluted with $\mathrm{CH}_{2} \mathrm{Cl}_{2}(10 \mathrm{~mL})$. The solvent was removed to obtain the crude product, which was analyzed using ${ }^{1} \mathrm{H}$ NMR using $\mathrm{CH}_{2} \mathrm{Br}_{2}(9.0 \mu \mathrm{L}, 0.125 \mathrm{mmol})$ as the internal standard. The enantiomeric ratio was determined by HPLC analysis on a chiral column. 


\section{Optimization Studies}

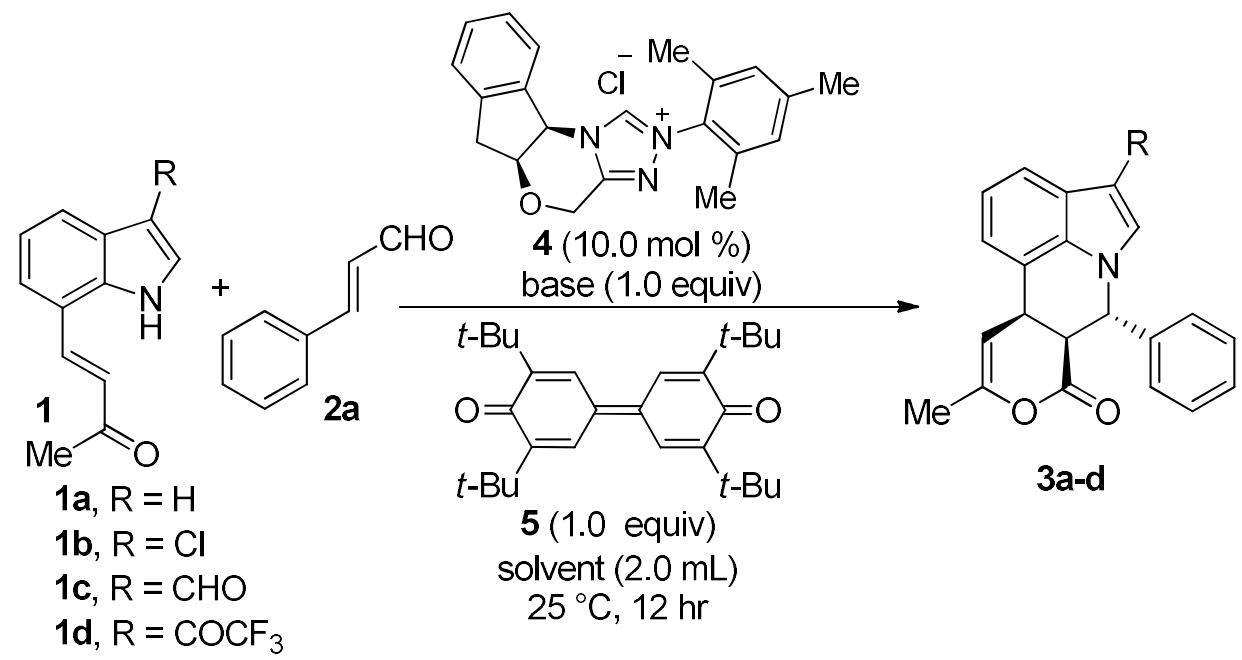

\begin{tabular}{|c|c|c|c|c|c|c|}
\hline Entry & (1) & Base & Solvent & $\begin{array}{l}\text { Yield } \\
(\%)^{b}\end{array}$ & $\mathrm{dr}^{\mathrm{c}}$ & $\mathrm{er}^{\mathrm{d}}$ \\
\hline 1 & $1 \mathrm{a}$ & DABCO & DMSO & $<5$ & -nd- & -nd- \\
\hline 2 & $1 \mathrm{~b}$ & $\mathrm{DABCO}$ & DMSO & $<5$ & -nd- & -nd- \\
\hline 3 & $1 \mathrm{c}$ & DABCO & DMSO & 42 & $>20: 1$ & $77: 23$ \\
\hline 4 & $1 d$ & DABCO & DMSO & 60 & $>20: 1$ & $95: 5$ \\
\hline 5 & $1 d$ & DABCO & toluene & 18 & $>20: 1$ & $56: 44$ \\
\hline 6 & $1 d$ & $\mathrm{DABCO}$ & $\mathrm{CH}_{2} \mathrm{Cl}_{2}$ & 18 & $>20: 1$ & $70: 30$ \\
\hline 7 & $1 d$ & DABCO & THF & 40 & $>20: 1$ & $73: 27$ \\
\hline 8 & $1 \mathrm{~d}$ & $\mathrm{DABCO}$ & DMF & 61 & $>20: 1$ & $93: 7$ \\
\hline 9 & $1 d$ & DABCO & $\mathrm{CHCl}_{3}$ & 31 & $>20: 1$ & $75: 25$ \\
\hline 10 & $1 d$ & DABCO & EtOAc & 36 & $>20: 1$ & $74: 26$ \\
\hline 11 & $1 d$ & DABCO & $\mathrm{CH}_{3} \mathrm{CN}$ & 69 & $>20: 1$ & 89:11 \\
\hline 12 & $1 d$ & DABCO & Acetone & 52 & $>20: 1$ & $90: 10$ \\
\hline 13 & $1 d$ & DABCO & DME & 18 & $>20: 1$ & $77: 23$ \\
\hline 14 & $1 \mathrm{~d}$ & DBU & DMSO & $<5$ & -nd- & -nd- \\
\hline 15 & $1 \mathrm{~d}$ & $\mathrm{~K}_{2} \mathrm{CO}_{3}$ & DMSO & 49 & $>20: 1$ & $95: 5$ \\
\hline 16 & $1 \mathrm{~d}$ & $\mathrm{Cs}_{2} \mathrm{CO}_{3}$ & DMSO & 47 & $>20: 1$ & $95: 5$ \\
\hline 17 & $1 \mathrm{~d}$ & DMAP & DMSO & 37 & $>20: 1$ & $95: 5$ \\
\hline $18^{\mathrm{d}}$ & $1 \mathrm{~d}$ & DABCO & DMSO & 84 & $>20: 1$ & 95:5 \\
\hline
\end{tabular}

${ }^{\mathrm{a}}$ Conditions: 1 ( $\left.0.125 \mathrm{mmol}\right), \mathbf{2 a}(0.125 \mathrm{mmol}), 4(10 \mathrm{~mol} \%), 5$ (1.0 equiv), base (1.0 equiv), solvent (1.0 $\mathrm{mL}), 25{ }^{\circ} \mathrm{C}$ and $12 \mathrm{~h}$. ${ }^{\mathrm{b}}$ The yields were determined by ${ }^{1} \mathrm{H}$ NMR analysis of crude product using $\mathrm{CH}_{2} \mathrm{Br}_{2}$ as the internal standard. ${ }^{\mathrm{c}}$ Diastereomeric ratio was determined by ${ }^{1} \mathrm{H}$ NMR spectroscopy prior to purification. ${ }^{\mathrm{d}}$ Enantiomer ratio was determined by HPLC analysis on a chiral column. ${ }^{\mathrm{e}} 1.5$ equiv each of 2a and $\mathbf{5}$, and $15 \mathrm{~mol} \%$ of $\mathbf{4}$ was used. 
The enhancement in reactivity and selectivity was observed while performing the reaction in polar aprotic solvents, such as $\mathrm{CH}_{3} \mathrm{CN}$, DMF, DMSO etc. To validate this, the reaction was performed in various solvents and the dielectric constant (DEC) of the solvent was plotted against the yield/ee of $\mathbf{3 d}$ obtained (Figure S1). ${ }^{4}$ For instance, the reaction afforded poor yield/ee when performed in toluene (DEC: 2.38), moderate yield/ee in THF (DEC: 7.58), and high yield/ee in DMF (DEC: 36.7). Although a general linear relationship was not observed, the overall trend observed was that the reactivity/selectivity was improved in polar aprotic solvents.

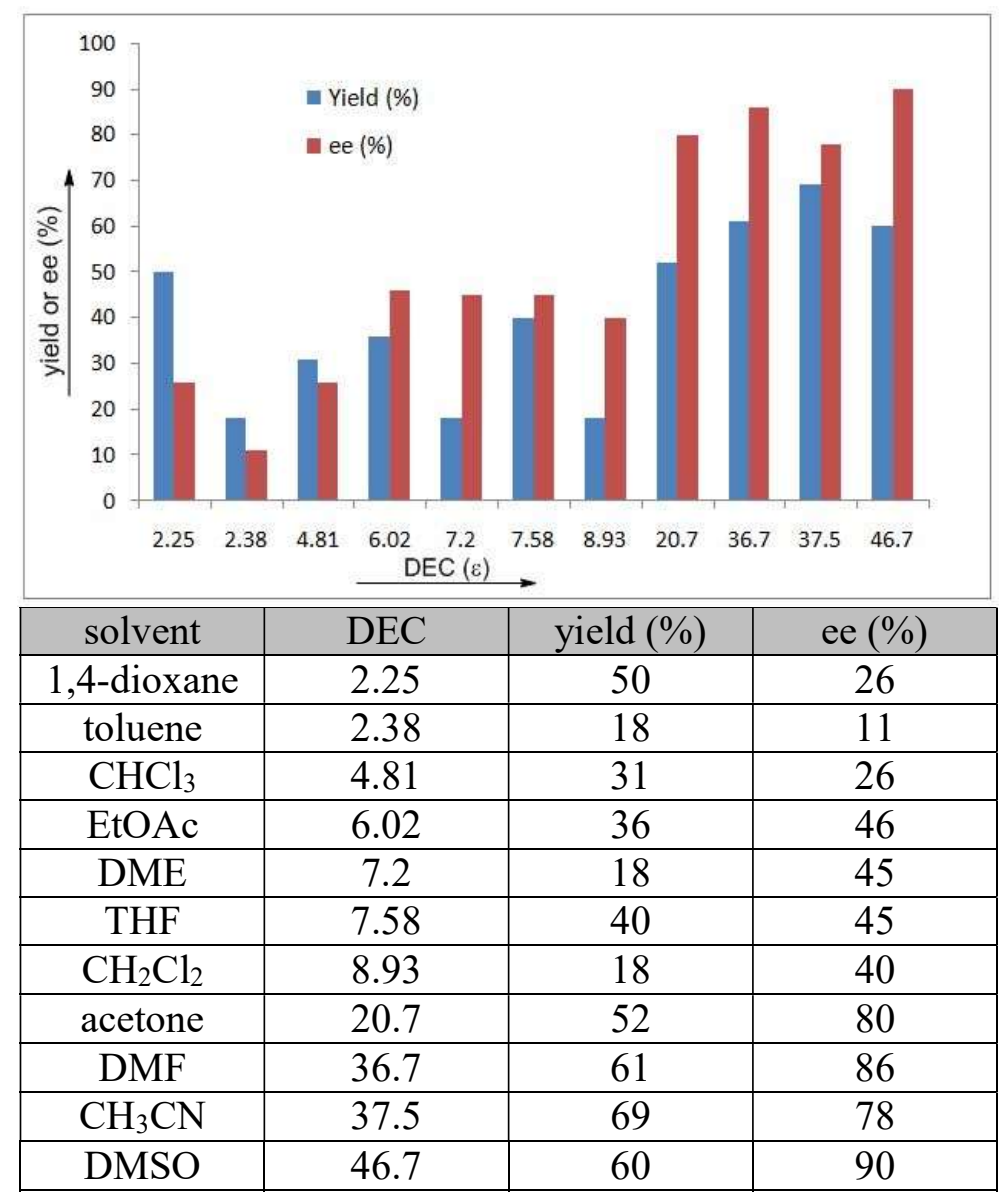

Figure S1. Variation of yield/ee of $\mathbf{3 d}$ with polarity of solvent (dielectric constant)

\footnotetext{
${ }^{4}$ Gorman, W. G.; Hall, G. D. J. Pharm. Sci. 1964, 53, 1017.
} 


\section{General Procedure for the Enantioselective Synthesis of Pyrroloquinolines}

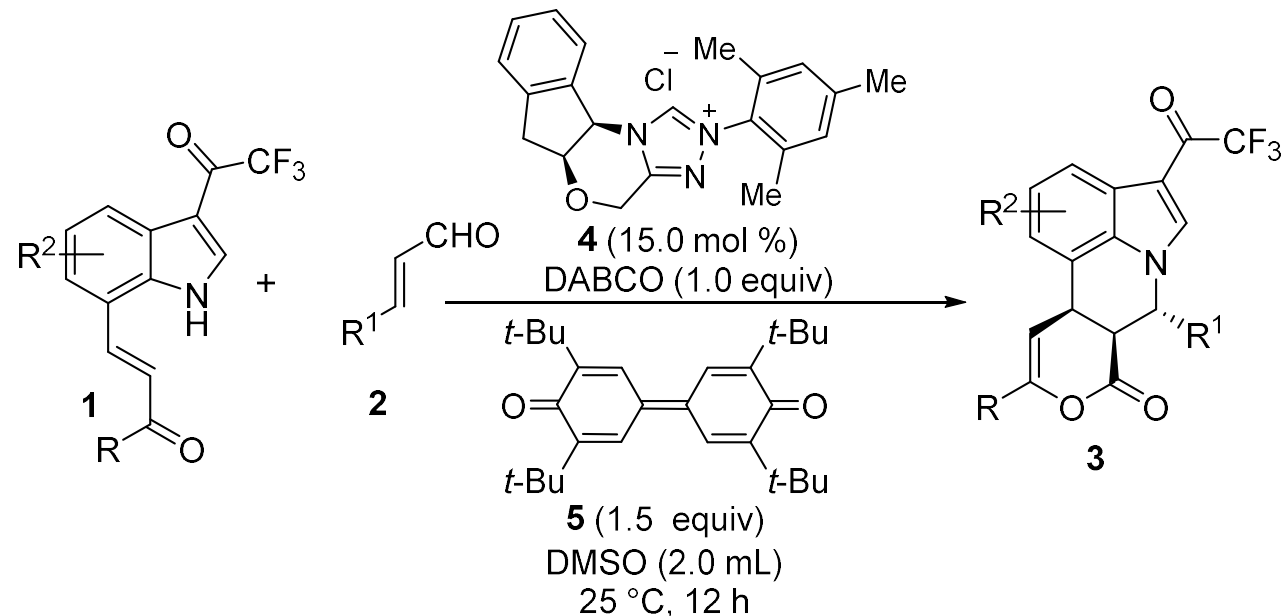

To a flame-dried screw-capped test tube equipped with a magnetic stir bar was added the triazolium salt $4(0.015 \mathrm{~g}, 0.037 \mathrm{mmol})$ and N-H indole derivative bearing a Michael acceptor moiety at the 7-position $1(0.25 \mathrm{mmol})$, and enal $2(0.375 \mathrm{mmol})$ was added. Then the screwcapped tube was evacuated and backfilled with argon. To this mixture was added DMSO (2.0 $\mathrm{mL})$ under argon atmosphere. To this mixture DABCO $(0.25 \mathrm{mmol})$ was successively added, and the resulting mixture was stirred at $25^{\circ} \mathrm{C}$ for $12 \mathrm{~h}$. When the reaction is complete, standard work-up of the reaction mixture was done using EtOAc and organic fractions were dried with $\mathrm{Na}_{2} \mathrm{SO}_{4}$, and solvent was evaporated. The crude residue was purified by flash column chromatography on silica gel to afford the corresponding pyrroloquinoline derivatives $\mathbf{3}$. All racemic pyrroloquinoline derivatives were prepared using NHC generated from the $N$-mesityl triazolium salt using DABCO.

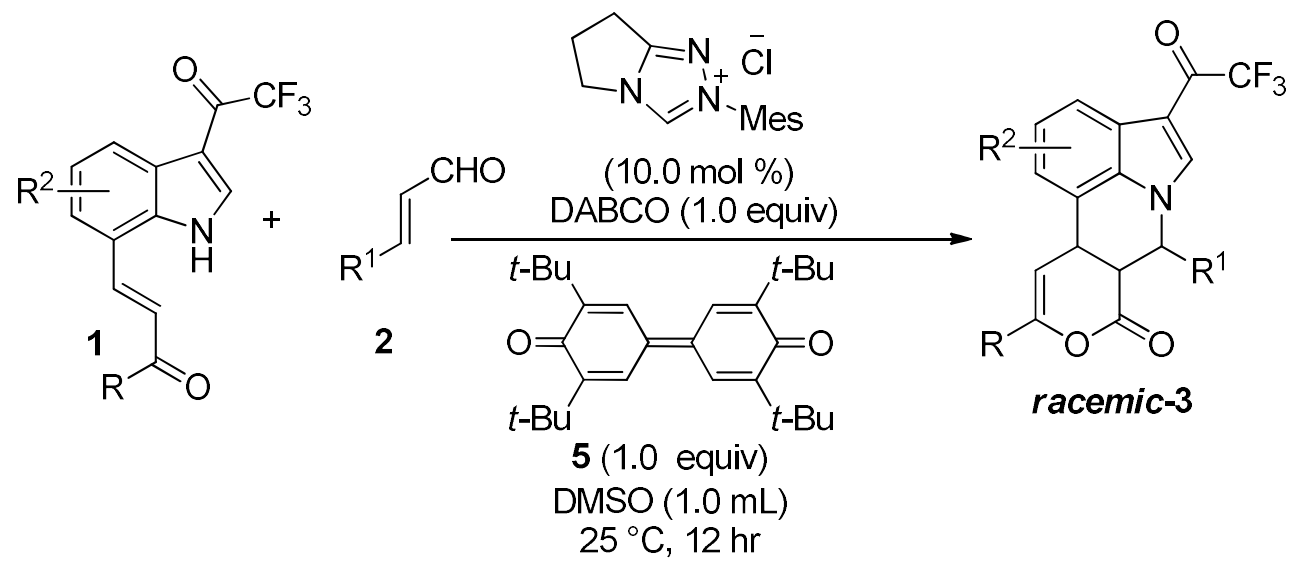




\section{Procedure for 1.0 mmol scale experiment}

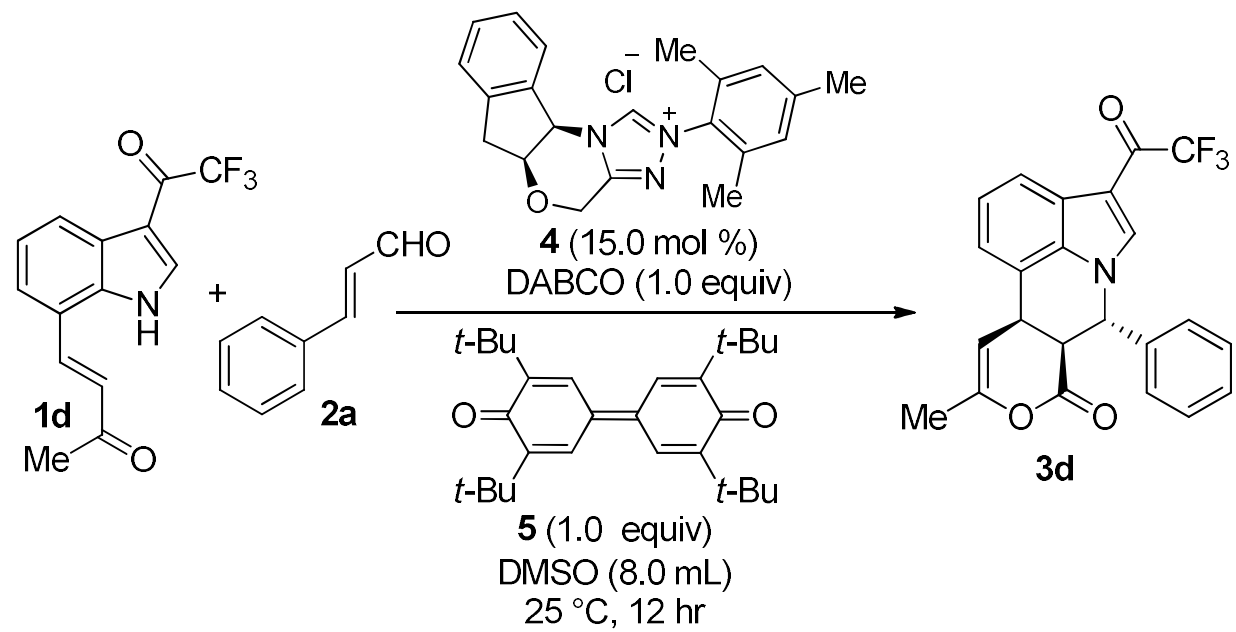

To a flame-dried screw-capped test tube equipped with a magnetic stir bar was added the triazolium salt $4(56.0 \mathrm{mg}, 0.15 \mathrm{mmol}, 15 \mathrm{~mol} \%)$ and $\mathrm{N}-\mathrm{H}$ indole derivative bearing a Michael acceptor moiety at the 7-position $\mathbf{1 d}(281.0 \mathrm{mg}, 1.0 \mathrm{mmol}, 1.0$ equiv) and enal 2a (198.0 mg, 1.5 mmol, 1.5 equiv) was added. Then the screw-capped tube was evacuated and backfilled with argon. To this mixture was added DMSO $(8.0 \mathrm{~mL})$ under argon atmosphere. To this mixture DABCO (112 mg, $1.0 \mathrm{mmol}, 1.0$ equiv) was successively added. and the resulting mixture was stirred at $25{ }^{\circ} \mathrm{C}$ for $12 \mathrm{~h}$. When the reaction is complete, standard work-up of the reaction mixture was done using EtOAc and organic fractions were dried with $\mathrm{Na}_{2} \mathrm{SO}_{4}$, solvent was evaporated. The crude residue was purified by flash column chromatography on silica gel to afford the corresponding pyrroloquinoline derivative $\mathbf{3 d}$ as white solid (353 $\mathrm{mg}, 86 \%$ yield, $>20: 1 \mathrm{dr}, 95: 5 \mathrm{er})$.

\section{X-ray Data of $3 h$}

Single crystal of $\mathbf{3 h}$ (recrystallized from 1:4 $\mathrm{CHCl}_{3} / n$-Hexane at $25^{\circ} \mathrm{C}$ ) was mounted and the diffraction data was collected at $296 \mathrm{~K}$ on a Bruker SMART APEX CCD diffractometer using SMART/SAINT software. Intensity data were collected using graphite-monochromatized Mo-Ka radiation $(71.073 \mathrm{pm})$. The structure was solved by direct methods using the SHELX-97 and refined by full-matrix least-squares on F2. Empirical absorption corrections were applied with SADABS. All Non-hydrogen atoms were refined anisotropically and hydrogen atoms were 
included in geometric positions. Structure was drawn using Olex-2 and ORTEP-3. The crystallographic refinement parameters are given below:

\begin{tabular}{|c|c|}
\hline Identification code & $\mathbf{3 h}$ \\
\hline CCDC & CCDC 1863110 \\
\hline Empirical formula & $\mathrm{C}_{23} \mathrm{H}_{15} \mathrm{ClF}_{3} \mathrm{NO}_{3}$ \\
\hline Formula weight & 445.81 \\
\hline Temperature/K & $296(2)$ \\
\hline Crystal system & orthorhombic \\
\hline Space group & $\mathrm{P} 2{ }_{1} 2{ }_{1}{ }_{1}$ \\
\hline $\mathrm{a} / \AA ̊$ & $10.3570(8)$ \\
\hline $\mathrm{b} / \AA$ & $17.0723(13)$ \\
\hline $\mathrm{c} / \AA$ & $23.2760(18)$ \\
\hline$\alpha /^{\circ}$ & 90 \\
\hline$\beta /{ }^{\circ}$ & 90 \\
\hline$\gamma /{ }^{\circ}$ & 90 \\
\hline Volume $/ \AA^{3}$ & $4115.6(5)$ \\
\hline $\mathrm{Z}$ & 8 \\
\hline$\rho_{\text {calc }} \mathrm{g} / \mathrm{cm}^{3}$ & 1.439 \\
\hline$\mu / \mathrm{mm}^{-1}$ & 0.238 \\
\hline $\mathrm{F}(000)$ & 1824.0 \\
\hline Crystal size $/ \mathrm{mm}^{3}$ & $0.25 \times 0.15 \times 0.12$ \\
\hline Radiation & $\operatorname{MoK} \alpha(\lambda=0.71073)$ \\
\hline \multicolumn{2}{|c|}{$2 \Theta$ range for data collection $/{ }^{\circ} 5.768$ to 55.19} \\
\hline Index ranges & $-13 \leq \mathrm{h} \leq 13,-22 \leq \mathrm{k} \leq 22,-30 \leq 1 \leq 30$ \\
\hline Reflections collected & 131106 \\
\hline Independent reflections & $9493\left[\mathrm{R}_{\text {int }}=0.0680, \mathrm{R}_{\text {sigma }}=0.0336\right]$ \\
\hline Data/restraints/parameters & $9493 / 0 / 561$ \\
\hline Goodness-of-fit on $\mathrm{F}^{2}$ & 1.031 \\
\hline Final $R$ indexes $[\mathrm{I}>=2 \sigma(\mathrm{I})]$ & $\mathrm{R}_{1}=0.0489, \mathrm{wR}_{2}=0.1313$ \\
\hline Final $\mathrm{R}$ indexes [all data] & $\mathrm{R}_{1}=0.0803, \mathrm{wR}_{2}=0.1523$ \\
\hline \multicolumn{2}{|c|}{ Largest diff. peak/hole / e $\AA^{-3} 0.34 /-0.30$} \\
\hline Flack parameter & $0.00(2)$ \\
\hline
\end{tabular}




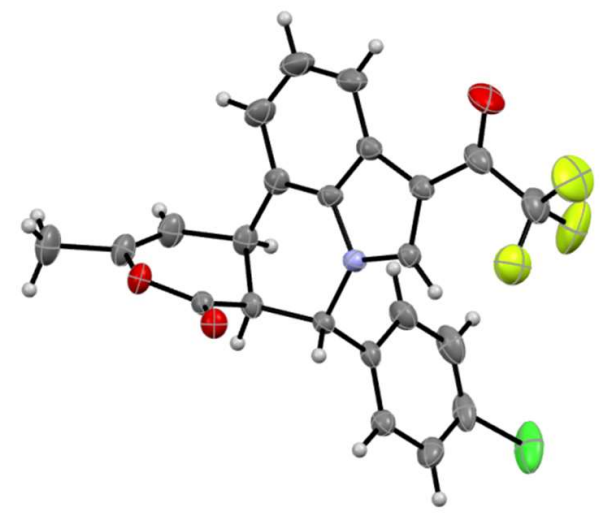

Figure S2. ORTEP representation of the X-ray structure of $\mathbf{3 h}$ (thermal ellipsoids at $30 \%$ probability)

\section{Synthesis and Characterization of Indole Derivatives}

The $1 H$-indole-7-carbaldehyde derivatives were prepared from following the known synthetic route depicted below. ${ }^{2}$ Next, 3-(2,2,2-trifluoroacetyl)-1H-indole-7-carbaldehyde derivatives and 1-(triphenylphosphoranylidene)propan-2-one were added to $\mathrm{CH}_{3} \mathrm{CN}$. The reaction mixture was reflux for overnight. The reaction mixture cooled and the solvent was evaporarated under reduced pressure. This was followed by purification using column chromatography to provide $N$-unsubstituted indole derivative bearing a Michael acceptor at the 7-position as a light green solid.
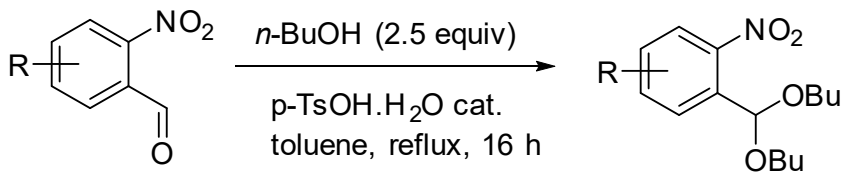

1) $\mathrm{MgBr}$ (3.3 equiv)

$\mathrm{THF},-45^{\circ} \mathrm{C}, 45 \mathrm{~min}$

1) $1 \mathrm{M} \mathrm{HCl}, \mathrm{rt}, 45 \mathrm{~min}$<smiles>[R]c1ccc2cc[nH]c2c1C=O</smiles><smiles>[R]C=CC1=CC=Cc2cc1ncc2C(=O)C(F)(F)F</smiles><smiles>CC(=O)C=P(c1ccccc1)(c1ccccc1)c1ccccc1</smiles>

$\left(\mathrm{CF}_{3} \mathrm{CO}\right)_{2} \mathrm{O}$

2.5 (equiv)

DMF, $16 \mathrm{~h}$

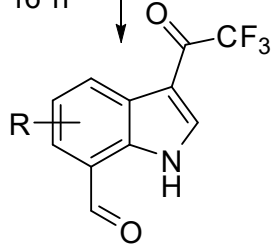




\section{(E)-7-(3-Oxobut-1-en-1-yl)-1H-indole-3-carbaldehyde (1c)}<smiles>CC(=O)/C=C/c1cccc2c(C=O)c[nH]c12</smiles>

Following the general procedure, ${ }^{2}$ treatment of $(E)-4-(1 H$-indol-7-yl)but-3-en2-one (348 mg, $1.878 \mathrm{mmol})$ and phosphoryl trichloride $(317 \mathrm{mg}, 2.066$ mmol) in DMF $(2.0 \mathrm{~mL})$ and stirring the reaction mixture at $25^{\circ} \mathrm{C}$ for $16 \mathrm{~h}$ followed by flash column chromatography (Pet. ether- EtOAc: 7:3) to afford (E) -7-(3-oxobut-1-en-1-yl)-1H-indole-3-carbaldehyde 1c as a white solid (151.0 $\mathrm{mg}, 37 \%$ yield).

$\boldsymbol{R}_{f}($ Pet. ether $/$ EtOAc $=70 / 30): 0.2$;

${ }^{1}$ H NMR (400 MHz, DMSO-d $\left.\boldsymbol{d}_{6}\right) \delta 12.68$ (s, 1H), 10.02 (s, 1H), 8.47(s, 1H), 8.23-8.17 (m, 2H), $7.77(\mathrm{~d}, J=7.3 \mathrm{~Hz}, 1 \mathrm{H}), 7.32$ (d, $J=7.5 \mathrm{~Hz}, 1 \mathrm{H}), 6.97$ (d, $J=162 \mathrm{~Hz}, 1 \mathrm{H}), 2.45$ (s, 3H). ${ }^{13} \mathbf{C}$ NMR (100 MHz, DMSO-d d $_{\text {d }}$ 198.17, 185.22, 139.00, 137.88, 135.91, 128.01, 125.12, 123.28, 122.68, 121.67, 119.36, 118.40, 27.37. HRMS (ESI) calculated $[\mathrm{M}+\mathrm{H}]^{+}$for $\mathrm{C}_{13} \mathrm{H}_{12} \mathrm{NO}_{2}$ : 214.0863,found: 214.0868. FTIR (cm $\left.{ }^{-1}\right)$ 3428, 2923, 2853, 2131, 1529, 1462, 1374, 1231, 1179 , 990.

\section{(E)-4-(3-(2,2,2-Trifluoroacetyl)-1H-indol-7-yl)but-3-en-2-one (1d)}<smiles>CC(=O)/C=C/c1cccc2c(C(=O)C(F)(F)F)c[nH]c12</smiles>

Following the known procedure, ${ }^{5}$ 3-(2,2,2-trifluoroacetyl)-1H-indole-7carbaldehyde (1.0 g, $4.14 \mathrm{mmol})$ and 1-(triphenylphosphoranylidene)propan-2one $(1.58 \mathrm{~g}, 4.9 \mathrm{mmol})$ were added to $\mathrm{CH}_{3} \mathrm{CN}$. The reaction mixture was reflux for overnight and concentrated followed by purified by column chromatography to provide $(E)$-4-(3-(2,2,2-trifluoroacetyl)-1 $H$-indol-7-yl)but-3-en-2-one (1d) as a light green solid $(0.91 \mathrm{~g}, 78 \%$ yield $)$.

$\boldsymbol{R}_{\boldsymbol{f}}($ Pet. ether $/$ EtOAc $=80 / 20)$ : 0.2; ${ }^{1}$ H NMR (400 MHz, DMSO-d $) \delta 13.18(\mathrm{~s}, 1 \mathrm{H}), 8.59(\mathrm{~s}$, $1 \mathrm{H}), 8.26-8.19(\mathrm{~m}, 2 \mathrm{H}), 7.81(\mathrm{~d}, J=7.5 \mathrm{~Hz}, 1 \mathrm{H}), 7.37$ (t, $J=7.7 \mathrm{~Hz}, 1 \mathrm{H}), 6.93$ (d, $J=16.2 \mathrm{~Hz}$, 1H), 2.42 (s, 3H). ${ }^{13}$ C NMR (100 MHz, DMSO-d 6 ) $\delta$ 198.14, 174.11 (q, $J=34.3 \mathrm{~Hz}$ ), 138.07 (q, $J=4.3 \mathrm{~Hz}), 137.27,135.54,128.53,126.68,123.89,123.34,122.04,120.03,116.78$ (q, $J=$ $290.0 \mathrm{~Hz}$ ), 109.17, 27.34. HRMS (ESI) calculated $[\mathrm{M}+\mathrm{H}]^{+}$for $\mathrm{C}_{14} \mathrm{H}_{11} \mathrm{O}_{2} \mathrm{~N}_{1} \mathrm{~F}_{3}: 282.0736$, found: 282.0745. FTIR (cm-1) 3157, 2912, 1667, 1580, 1522, 1463, 1271, 1232, 1125, 956, 889. 
(E)-1-Cyclopropyl-3-(3-(2,2,2-trifluoroacetyl)-1H-indol-7-yl)prop-2-en-1-one (1w)<smiles>N#C/C(=C\c1cccc2c(C(=O)C(F)(F)F)c[nH]c12)C1CC1</smiles>

Following the known procedure, ${ }^{5}$ 3-(2,2,2-trifluoroacetyl)-1 $H$-indole-7carbaldehyde $(0.2 \mathrm{~g}, \quad 0.82 \mathrm{mmol})$ and 1-cyclopropyl-2-(triphenyl phosphoranylidene)ethanone $(0.36 \mathrm{~g}, 1.0 \mathrm{mmol})$ were added to $\mathrm{CH}_{3} \mathrm{CN}$. The reaction mixture was reflux for over night and concentrated followed by purified by column chromatography to provide $(E)$-1-cyclopropyl-3-(3-(2,2,2trifluoroacetyl)-1H-indol-7-yl)prop-2-en-1-one (1w) as a white solid $(0.21 \mathrm{~g}$, $83 \%$ yield).

$\boldsymbol{R}_{\boldsymbol{f}}($ Pet. ether $/$ EtOAc $=80 / 20): 0.4 ;{ }^{1} \mathbf{H}$ NMR (400 MHz, $\left.\mathbf{C D C l}_{3}\right) \delta 10.84(\mathrm{~s}, 1 \mathrm{H}), 8.48(\mathrm{~d}, J=$ $7.9 \mathrm{~Hz}, 1 \mathrm{H}), 8.21-8.13(\mathrm{~m}, 2 \mathrm{H}), 7.68(\mathrm{~d}, J=7.4 \mathrm{~Hz}, 1 \mathrm{H}), 7.41$ (t, $J=7.7 \mathrm{~Hz}, 1 \mathrm{H}), 7.11(\mathrm{~d}, J=$ $15.7 \mathrm{~Hz}, 1 \mathrm{H}), 2.28-2.25(\mathrm{~m}, 1 \mathrm{H}), 1.24-1.12(\mathrm{~m}, 4 \mathrm{H}) .{ }^{13} \mathbf{C}$ NMR (100 MHz, CDCl $) \delta 201.36$, $175.62(\mathrm{q}, J=35.2 \mathrm{~Hz}), 136.97,135.60,135.20$ (q, $J=5.2 \mathrm{~Hz}), 127.19,126.76,125.11,124.24$, 123.37, 119.68, 117.07 (q, $J=291.0 \mathrm{~Hz}$ ), 111.32, 21.53, 12.33. HRMS (ESI) calculated [M+H] for $\mathrm{C}_{16} \mathrm{H}_{13} \mathrm{O}_{2} \mathrm{~N}_{1} \mathrm{~F}_{3}$ : 308.0893, found: 308.0895. FTIR (cm-1) 3361, 2913, 1664, 1591, 1447, $1390,1272,1181,1130,973,888$.

\section{(E)-5-Methyl-1-(3-(2,2,2-trifluoroacetyl)-1H-indol-7-yl)hex-1-en-3-one (1x)}<smiles>CC(C)CC(=O)/C=C/c1cccc2c(C(=O)C(F)(F)F)c[nH]c12</smiles>

$1 x$

Following the known procedure, ${ }^{5}$ treatment of 3-(2,2,2-trifluoroacetyl)- $1 \mathrm{H}$ indole-7-carbaldehyde (250 mg, $1.036 \mathrm{mmol})$ and 4-methyl-1-(triphenyl-15phosphaneylidene)pentan-2-one (448 $\mathrm{mg}, 1.244 \mathrm{mmol})$ in acetonitrile (5.0 $\mathrm{mL}$ ) and stirring the reaction mixture in reflux for $12 \mathrm{~h}$, followed by flash column chromatography (Pet. ether- EtOAc: 9:1) to afford (E)-5-methyl-1(3-(2,2,2-trifluoroacetyl)-1H-indol-7-yl)hex-1-en-3-one $\mathbf{1 x}$ as a green solid (291.0 mg, 87\% yield).

$\boldsymbol{R}_{\boldsymbol{f}}($ Pet. ether $/$ EtOAc $=90 / 10): 0.45 ;{ }^{1} \mathrm{H}$ NMR (400 MHz, DMSO-d $\left.)\right) \delta 13.20(\mathrm{~s}, 1 \mathrm{H}), 8.58(\mathrm{~s}$, 1H), 8.26 (d, $J=7.9 \mathrm{~Hz}, 1 \mathrm{H}), 8.20$ (d, $J=16.1 \mathrm{~Hz}, 1 \mathrm{H}), 7.85$ (d, $J=7.4 \mathrm{~Hz}, 1 \mathrm{H}), 7.39$ (t , $J=7.8$ $\mathrm{Hz}, 1 \mathrm{H}), 7.03(\mathrm{~d}, J=16.1 \mathrm{~Hz}, 1 \mathrm{H}), 2.66(\mathrm{~d}, J=6.9 \mathrm{~Hz}, 2 \mathrm{H}), 2.16(\mathrm{~m}, 1 \mathrm{H}), 0.95(\mathrm{~d}, J=6.7 \mathrm{~Hz}$, 6H). ${ }^{13}$ C NMR (100 MHz, DMSO-d $) \delta 199.75,174.10$ (q, $J=34.3 \mathrm{~Hz}$ ), 137.96 (q, $J=4.89$ Hz), 136.01, 135.58, 128.18, 126.69, 123.88, 123.29, 122.05, 120.12, 117.18, 109.16, 48.69,

\footnotetext{
${ }^{5}$ Mukherjee, S.; Ghosh, A.; Marelli, U. K.; Biju, A. T. Org. Lett. 2018, 20, 2952.
} 
24.53, 22.48. HRMS (ESI) calculated $[\mathrm{M}+\mathrm{H}]^{+}$for $\mathrm{C}_{17} \mathrm{H}_{17} \mathrm{~F}_{3} \mathrm{NO}_{2}$ : 324.1206, found: 324.1209 . FTIR (cm $\left.{ }^{-1}\right)$ 3359, 2956, 1658, 1528, 1461, 1369, 1297, 1264, 1180, 1130, 976.

\section{(E)-4-(5-Bromo-3-(2,2,2-trifluoroacetyl)-1H-indol-7-yl)but-3-en-2-one (1y)}<smiles>CC(=O)/C=C/c1cc(Br)cc2c(C(=O)C(F)(F)F)c[nH]c12</smiles>

Following the known procedure, ${ }^{5}$ 5-bromo-3-(2,2,2-trifluoroacetyl)- $1 \mathrm{H}$ indole-7-carbaldehyde $(0.44 \mathrm{~g}, 1.38 \mathrm{mmol})$ and 1-(triphenyl phosphoranylidene)propan-2-one $(0.52 \mathrm{~g}, 1.65 \mathrm{mmol})$ were added to $\mathrm{CH}_{3} \mathrm{CN}$. The reaction mixture was reflux for overnight and concentrated followed by purified by column chromatography to provide (E)-4-(5bromo-3-(2,2,2-trifluoroacetyl)-1H-indol-7-yl)but-3-en-2-one (1y) as a light green solid ( $0.4 \mathrm{~g}, 80 \%$ yield $)$.

$\boldsymbol{R}_{\boldsymbol{f}}($ Pet. ether $/$ EtOAc $=80 / 20): 0.35 ;{ }^{1}$ H NMR (400 MHz, DMSO-d $\left.\boldsymbol{d}_{\boldsymbol{6}}\right) \delta 13.31(\mathrm{~s}, 1 \mathrm{H}), 8.60(\mathrm{~s}$, 1H), $8.29(\mathrm{~d}, J=1.7 \mathrm{~Hz}, 1 \mathrm{H}), 8.08(\mathrm{~d}, J=16.2 \mathrm{~Hz}, 1 \mathrm{H}), 7.93$ (d, $J=1.6 \mathrm{~Hz}, 1 \mathrm{H}), 7.02$ (d, $J=$ $16.2 \mathrm{~Hz}, 1 \mathrm{H}), 2.40$ (s, 3H). ${ }^{13} \mathrm{C}$ NMR (100 MHz, DMSO-d 6 ) $\delta 198.00,174.07$ (q, $J=35.1 \mathrm{~Hz}$ ), $138.81(\mathrm{q}, J=4.5 \mathrm{~Hz}), 135.46,134.37,129.75,128.20,124.86,124.05,121.94,116.65,116.60$ (q, $J=292.0 \mathrm{~Hz}), 108.58,27.60$. HRMS (ESI) calculated $[\mathrm{M}+\mathrm{H}]^{+}$for $\mathrm{C}_{14} \mathrm{H}_{10} \mathrm{O}_{2} \mathrm{~N}_{1} \mathrm{~F}_{3} \mathrm{Br}$ : 359.9842, found: 359.9847. FTIR (cm $\left.\mathbf{( c m}^{-1}\right)$ 3398, 3135, 2912, 1662, 1589, 1516, 1451, 1356, 1124, $976,869$.

\section{Synthesis and Characterization of Pyrroloquinoline Derivatives}

\section{(7S,7aS,11aR)-10-Methyl-8-oxo-7-phenyl-7a,11a-dihydro-7H,8H-pyrano[3,4-}

c]pyrrolo[3,2,1-ij]quinoline-4-carbaldehyde (3c)<smiles>CC1=C[C@H]2c3cccc4c(C=O)cn(c34)[C@H](c3ccccc3)[C@H]2C(=O)O1</smiles>

Following the general procedure, treatment of cinnamaldehyde $\mathbf{2 a}$ (37.0 $\mathrm{mg}, 35 \mu \mathrm{L}, 0.280 \mathrm{mmol})$ and (E)-7-(3-oxobut-1-en-1-yl)-1H-indole-3carbaldehyde 1c $(40.0 \mathrm{mg}, 0.187 \mathrm{mmol})$ with triazolium salt $4(10.3 \mathrm{mg}$, $0.0280 \mathrm{mmol})$, oxidant 5 (114.4 $\mathrm{mg}, 0.280 \mathrm{mmol})$ and DABCO (20.9 mg, $0.187 \mathrm{mmol})$ in DMSO $(2.0 \mathrm{~mL})$ and stirring the reaction mixture at 25 ${ }^{\circ} \mathrm{C}$ for $12 \mathrm{~h}$ followed by flash column chromatography (Pet. ether- 
EtOAc: $7: 3)$ to afford $(7 S, 7 \mathrm{a} S, 11 \mathrm{a} R)$-10-methyl-8-oxo-7-phenyl-7a,11a-dihydro-7H,8Hpyrano[3,4-c]pyrrolo[3,2,1-ij]quinoline-4-carbaldehyde $\mathbf{3 c}$ as a brown solid $(27.0 \mathrm{mg}, 42 \%$ yield).

$\boldsymbol{R}_{\boldsymbol{f}}($ Pet. ether $/$ EtOAc $=70 / 30): 0.30$; er $=77: 23,[\alpha]_{\mathrm{D}}{ }^{25}=+118.7\left(\mathrm{c} 0.2, \mathrm{CHCl}_{3}\right)$. HPLC (Chiralpak AD, 80:20 Hexane / IPA, 1.0 mL/min) Minor: $11.6 \mathrm{~min}$, Major: $13.6 \mathrm{~min}$.

${ }^{1}$ H NMR (400 MHz, CDCl $) \delta 10.00(\mathrm{~s}, 1 \mathrm{H}), 8.16$ (d, $\left.J=7.8 \mathrm{~Hz}, 1 \mathrm{H}\right), 7.78(\mathrm{~s}, 1 \mathrm{H}), 7.33-7.31$ (m, 4H), $7.12(\mathrm{~d}, J=7.2 \mathrm{~Hz}, 1 \mathrm{H}), 6.82(\mathrm{~s}, 1 \mathrm{H}), 6.30(\mathrm{~s}, 1 \mathrm{H}), 5.64(\mathrm{~d}, J=6.7 \mathrm{~Hz}, 1 \mathrm{H}), 3.70$ (t, $J=$ $6.1 \mathrm{~Hz}, 1 \mathrm{H}), 3.37(\mathrm{~d}, J=6.3 \mathrm{~Hz}, 1 \mathrm{H}), 1.94(\mathrm{~s}, 3 \mathrm{H}) \cdot{ }^{13} \mathbf{C}$ NMR (100 MHz, CDCl$)_{3} \delta 184.02$, $166.66,149.21$, 138.36, 136.09, 133.00, 128.44, 127.77, 124.50, 122.87, 122.14, 120.09, 119.98, 119.92, 118.77, 101.17, 55.94, 44.85, 26.33, 17.76. HRMS (ESI) calculated $[\mathrm{M}+\mathrm{H}]^{+}$for $\mathrm{C}_{22} \mathrm{H}_{18} \mathrm{O}_{3} \mathrm{~N}:$ 344.1281, found: 344.1290. FTIR (cm-1) 3406, 3103, 2923, 2807, 1761, 1659, $1522,1451,1150,1075,935$.

(7S,7aS,11a $R$ )-10-Methyl-7-phenyl-4-(2,2,2-trifluoroacetyl)-7,7a-dihydropyrano[3,4c]pyrrolo[3,2,1-ij]quinolin-8(11aH)-one (3d)<smiles>CC1=CC2c3cccc4c(C(=O)C(F)(F)F)cn(c34)[C@@H](c3ccccc3)[C@H]2C(=O)O1</smiles>

Following the general procedure, treatment of cinnamaldehyde 2a (50.0 $\mathrm{mg}, 47 \mu \mathrm{L}, 0.375 \mathrm{mmol})$ and $(E)-4-(3-(2,2,2$-trifluoroacetyl)-1H-indol-7yl)but-3-en-2-one $1 \mathbf{d}(70.0 \mathrm{mg}, 0.25 \mathrm{mmol})$ with triazolium salt $4(15.0$ $\mathrm{mg}, 0.037 \mathrm{mmol})$, oxidant 5 (153.0 $\mathrm{mg}, 0.375 \mathrm{mmol})$ and DABCO (28.0 $\mathrm{mg}, 0.25 \mathrm{mmol})$ in DMSO $(2.0 \mathrm{~mL})$ and stirring the reaction mixture at 25 ${ }^{\circ} \mathrm{C}$ for $12 \mathrm{~h}$ followed by flash column chromatography (Pet. ether- EtOAc: 9:1) to afford (7S,7aS,11aR)-10-methyl-7-phenyl-4-(2,2,2-trifluoroacetyl)-7,7adihydropyrano[3,4-c]pyrrolo[3,2,1-ij]quinolin-8(11aH)-one 3d as a white solid $(86.0 \mathrm{mg}, 84 \%$ yield).

$\boldsymbol{R}_{\boldsymbol{f}}($ Pet. ether $/$ EtOAc $=80 / 20): 0.50$; er $=95: 5,[\alpha]_{\mathrm{D}}{ }^{25}=+156.6\left(\mathrm{c} 0.1, \mathrm{CHCl}_{3}\right)$. HPLC $($ Chiralpak AD, 90:10 Hexane / IPA, $1.0 \mathrm{~mL} / \mathrm{min}$ ) Minor: 7.7 min, Major: $9.9 \mathrm{~min}$.

${ }^{1}$ H NMR (400 MHz, CDCl$) \delta 8.25(\mathrm{~d}, J=8.0 \mathrm{~Hz}, 1 \mathrm{H}), 8.02(\mathrm{~s}, 1 \mathrm{H}), 7.40-7.33(\mathrm{~m}, 4 \mathrm{H}), 7.18$ $(\mathrm{d}, J=7.3 \mathrm{~Hz}, 1 \mathrm{H}), 6.82(\mathrm{~d}, J=4.0 \mathrm{~Hz}, 2 \mathrm{H}), 6.36(\mathrm{~s}, 1 \mathrm{H}), 5.66(\mathrm{~d}, J=6.8 \mathrm{~Hz}, 1 \mathrm{H}), 3.71(\mathrm{t}, J=$ $6.1 \mathrm{~Hz}, 1 \mathrm{H}), 3.40$ (d, $J=6.2 \mathrm{~Hz}, 1 \mathrm{H}), 1.95$ (s, 3H). ${ }^{13} \mathbf{C} \mathbf{N M R}\left(\mathbf{1 0 0} \mathbf{M H z}, \mathbf{C D C l}_{3}\right) \delta 175.35$ (q, $J$ $=35.1 \mathrm{~Hz}), 167.52,150.41,138.85,136.08(\mathrm{q}, J=4.8 \mathrm{~Hz}), 133.37,129.52,128.90,125.32$, 124.84, 121.64, 121.48, 121.39, 116.99 (q, $J=291.0 \mathrm{~Hz}$ ), 111.22, 101.92, 57.26, 45.81, 27.11, 
18.69. HRMS (ESI) calculated $[\mathrm{M}+\mathrm{H}]^{+}$for $\mathrm{C}_{23} \mathrm{H}_{17} \mathrm{O}_{3} \mathrm{~N}_{1} \mathrm{~F}_{3}$ : 412.1155, found: 412.1161. FTIR $\left(\mathbf{c m}^{-1}\right)$ 3412, 2923, 1763, 1672, 1517, 1393, 1146, 1048, 906.

(7S,7aS,11aR)-7-(4-(Dimethylamino)phenyl)-10-methyl-4-(2,2,2-trifluoroacetyl)-7,7adihydropyrano[3,4-c]pyrrolo[3,2,1-ij]quinolin-8(11aH)-one (3e)<smiles>CC1=CC2c3cccc4c(C(=O)C(F)(F)F)cn(c34)[C@H](c3ccc(N(C)C)cc3)C2C(=O)O1</smiles>

Following the general procedure, treatment of (E)-3-(4(dimethylamino)phenyl)acrylaldehyde 2 e $(66.0 \mathrm{mg}, 0.375 \mathrm{mmol})$ and (E)-4-(3-(2,2,2-trifluoroacetyl)-1H-indol-7-yl)but-3-en-2-one 1d (70.0 $\mathrm{mg}, 0.25 \mathrm{mmol})$ with triazolium salt $4(15.0 \mathrm{mg}, 0.037 \mathrm{mmol})$, oxidant $5(153.0 \mathrm{mg}, 0.375 \mathrm{mmol})$ and $\mathrm{DABCO}(28.0 \mathrm{mg}, 0.25 \mathrm{mmol})$ in DMSO $(2.0 \mathrm{~mL})$ and stirring the reaction mixture at $25^{\circ} \mathrm{C}$ for $12 \mathrm{~h}$ followed by flash column chromatography (Pet. ether- EtOAc: 9:1) to afford (7S,7aS,11aR)-7(4-(dimethylamino)phenyl)-10-methyl-4-(2,2,2-trifluoroacetyl)-7,7a-dihydropyrano[3,4c]pyrrolo[3,2,1-ij]quinolin-8(11a $H)$-one 3a as a black solid (70.0 $\mathrm{mg}, 63 \%$ yield). $\boldsymbol{R}_{\boldsymbol{f}}($ Pet. ether $/$ EtOAc $=80 / 20): 0.40 ;$ er $=98: 2,[\alpha]_{\mathrm{D}}{ }^{25}=+276.1\left(\mathrm{c} 0.1, \mathrm{CHCl}_{3}\right)$. HPLC $($ Chiralpak AD, 90:10 Hexane / IPA, $1.0 \mathrm{~mL} / \mathrm{min}$ ) Minor: $14.6 \mathrm{~min}$, Major: $11.3 \mathrm{~min}$.

${ }^{1}$ H NMR (400 MHz, CDCl $) \delta 8.24(\mathrm{~d}, J=7.9 \mathrm{~Hz}, 1 \mathrm{H}), 8.0(\mathrm{~s}, 1 \mathrm{H}), 7.36(\mathrm{t}, J=7.7 \mathrm{~Hz}, 1 \mathrm{H})$, $7.17(\mathrm{~d}, J=7.2 \mathrm{~Hz}, 1 \mathrm{H}), 6.72-6.65(\mathrm{~m}, 4 \mathrm{H}), 6.23(\mathrm{~s}, 1 \mathrm{H}), 5.64(\mathrm{~d}, J=6.7 \mathrm{~Hz}, 1 \mathrm{H}), 3.78(\mathrm{t}, J=$ $6.3 \mathrm{~Hz}, 1 \mathrm{H}), 3.34$ (d, $J=6.3 \mathrm{~Hz}, 1 \mathrm{H}), 2.93(\mathrm{~s}, 6 \mathrm{H}), 1.94(\mathrm{~s}, 3 \mathrm{H}) .{ }^{13} \mathbf{C}$ NMR (100 MHz, CDCl $) \delta$ 175.32 (q, $J=35.6 \mathrm{~Hz}), 167.76,150.42,150.25,136.21$ (q, $J=5.3 \mathrm{~Hz}), 133.46,126.31,124.88$, $124.71,121.51,121.36,118.47,115.58,112.93$, 110.96, 102.11, 56.85, 45.99, 40.54, 27.30, 18.73. HRMS (ESI) calculated $[\mathrm{M}+\mathrm{H}]^{+}$for $\mathrm{C}_{25} \mathrm{H}_{22} \mathrm{O}_{3} \mathrm{~N}_{2} \mathrm{~F}_{3}: 455.1577$, found: 455.1586. FTIR $\left(\mathbf{c m}^{-1}\right)$ 3428, 1763, 1670, 1615, 1519, 1451, 1391, 1143, 1047, 905.

(7S,7aS,11aR)-7-(4-Methoxyphenyl)-10-methyl-4-(2,2,2-trifluoroacetyl)-7,7adihydropyrano[3,4-c]pyrrolo[3,2,1-ij]quinolin-8(11aH)-one (3f)<smiles>COc1ccc([C@H]2[C@H]3C(=O)OC(C)=C[C@H]3c3cccc4c(C(=O)C(F)(F)F)cn2c34)cc1</smiles>

Following the general procedure, treatment of $(E)-3-(4-$ methoxyphenyl)acrylaldehyde $\mathbf{2 f}(61.0 \mathrm{mg}, 0.375 \mathrm{mmol})$ and $(E)-4-(3-$ (2,2,2-trifluoroacetyl)-1H-indol-7-yl)but-3-en-2-one 1d (70.0 mg, 0.25 mmol) with triazolium salt 4 (15.0 $\mathrm{mg}, 0.037 \mathrm{mmol})$, oxidant 5 (153.0 $\mathrm{mg}, 0.375 \mathrm{mmol})$ and DABCO $(28.0 \mathrm{mg}, 0.25 \mathrm{mmol})$ in DMSO $(2.0$ 
$\mathrm{mL}$ ) and stirring the reaction mixture at $25{ }^{\circ} \mathrm{C}$ for $12 \mathrm{~h}$ followed by flash column chromatography (Pet. ether- EtOAc: 9:1) to afford (7S,7aS,11aR)-7-(4-methoxyphenyl)-10methyl-4-(2,2,2-trifluoroacetyl)-7,7a-dihydropyrano[3,4-c]pyrrolo[3,2,1-ij]quinolin-8(11a $H)$-one 3f as a white solid ( $93.0 \mathrm{mg}, 84 \%$ yield).

$\boldsymbol{R}_{\boldsymbol{f}}($ Pet. ether $/ \mathrm{EtOAc}=80 / 20): 0.35$; er $=98: 2,[\alpha]_{\mathrm{D}}{ }^{25}=+159.6\left(\mathrm{c} 0.1, \mathrm{CHCl}_{3}\right)$. HPLC $($ Chiralpak IA, 90:10 Hexane / IPA, $1.0 \mathrm{~mL} / \mathrm{min}$ ) Minor: $12.7 \mathrm{~min}$, Major: $14.0 \mathrm{~min}$.

${ }^{1}$ H NMR (400 MHz, CDCl $) \delta 8.24(\mathrm{~d}, J=8.0 \mathrm{~Hz}, 1 \mathrm{H}), 8.01(\mathrm{~d}, J=1.1 \mathrm{~Hz}, 1 \mathrm{H}), 7.36(\mathrm{t}, J=7.7$ $\mathrm{Hz}, 1 \mathrm{H}), 7.18$ (d, $J=7.4 \mathrm{~Hz}, 1 \mathrm{H}), 6.84$ (d, $J=8.7 \mathrm{~Hz}, 2 \mathrm{H}), 6.75$ (d, $J=8.7 \mathrm{~Hz}, 2 \mathrm{H}), 6.29$ (s, 1H), $5.65(\mathrm{~d}, J=6.9 \mathrm{~Hz}, 1 \mathrm{H}), 3.76-3.72(\mathrm{~m}, 4 \mathrm{H}), 3.37\left(\mathrm{dd}, J_{l}=6.4, J_{2}=2,2 \mathrm{~Hz}, 1 \mathrm{H}\right), 1.94(\mathrm{~s}, 3 \mathrm{H}) .{ }^{13} \mathrm{C}$ NMR (100 MHz, $\left.\mathbf{C D C l}_{3}\right) \delta 175.30(\mathrm{q}, J=36.1 \mathrm{~Hz}), 167.57,159.96,150.33,136.06(\mathrm{q}, J=4.9$ Hz), 133.34, 130.63, 126.61, 124.84, 121.60, 121.41, 117.0 (q, $J=290.0 \mathrm{~Hz}), 114.83,111.10$, 101.98, 56.77, 56.41, 45.87, 27.17, 18.66. HRMS (ESI) calculated $[\mathrm{M}+\mathrm{H}]^{+}$for $\mathrm{C}_{24} \mathrm{H}_{19} \mathrm{O}_{4} \mathrm{~N}_{1} \mathrm{~F}_{3}$ : 442.1261, found: 442.1267. FTIR (cm-1) 3444, 2927, 1763, 1672, 1515, 1391, 1254, 1177, 1047, 906.

(7S,7aS,11a R)-10-Methyl-7-(p-tolyl)-4-(2,2,2-trifluoroacetyl)-7a,11a-dihydro-7H,8Hpyrano[3,4-c]pyrrolo[3,2,1-ij]quinolin-8-one (3g)<smiles>CC1=C[C@H]2c3cccc4c(C(=O)C(F)(F)F)cn(c34)[C@H](c3ccc(C)cc3)[C@H]2C(=O)O1</smiles>

Following the general procedure, treatment of $(E)-3-(\mathrm{p}-$ tolyl)acrylaldehyde $2 \mathrm{~g}(54.8 \mathrm{mg}, 0.375 \mathrm{mmol})$ and $(E)-4-(3-(2,2,2-$ trifluoroacetyl)-1H-indol-7-yl)but-3-en-2-one $1 d$ (70.0 mg, 0.25 mmol) with triazolium salt $4(15.0 \mathrm{mg}, 0.037 \mathrm{mmol})$, oxidant 5 $(153.0 \mathrm{mg}, 0.375 \mathrm{mmol})$ and DABCO $(28.0 \mathrm{mg}, 0.25 \mathrm{mmol})$ in DMSO $(2.0 \mathrm{~mL})$ and stirring the reaction mixture at $25^{\circ} \mathrm{C}$ for $12 \mathrm{~h}$ followed by flash column chromatography (Pet. ether- EtOAc: 9:1) to afford (7S,7aS,11aR)-10methyl-7-phenyl-4-(2,2,2-trifluoroacetyl)-7,7a-dihydropyrano[3,4-c]pyrrolo[3,2,1-ij]quinolin$8(11 \mathrm{a} H)$-one $3 \mathrm{~g}$ as a brown solid (100.0 $\mathrm{mg}, 93 \%$ yield).

$\boldsymbol{R}_{\boldsymbol{f}}($ Pet. Ether $/$ EtOAc $=80 / 20): 0.46$; er $=96: 4,[\alpha]_{\mathrm{D}}{ }^{25}=138.88\left(\right.$ c 1.0, $\left.\mathrm{CHCl}_{3}\right)$. HPLC $($ Chiralpak AD, 90:10 Hexane / IPA, $1.0 \mathrm{~mL} / \mathrm{min}$ ) Minor: $8.9 \mathrm{~min}$, Major: $10.1 \mathrm{~min}$.

${ }^{1}$ H NMR (400 MHz, CDCl $) \delta 8.25(\mathrm{~d}, J=8.0 \mathrm{~Hz}, 1 \mathrm{H}), 8.02(\mathrm{~s}, 1 \mathrm{H}), 7.37$ (t, $\left.J=7.7 \mathrm{~Hz}, 1 \mathrm{H}\right)$, $7.18(\mathrm{~d}, J=7.4 \mathrm{~Hz}, 1 \mathrm{H}), 7.13(\mathrm{~d}, J=8.0 \mathrm{~Hz}, 2 \mathrm{H}), 6.72(\mathrm{~d}, J=8 \mathrm{~Hz}, 2 \mathrm{H}), 6.32(\mathrm{~s}, 1 \mathrm{H}), 5.66(\mathrm{~d}, J$ $=7 \mathrm{~Hz}, 1 \mathrm{H}), 3.73(\mathrm{t}, J=6.6 \mathrm{~Hz}, 1 \mathrm{H}), 3.39\left(\mathrm{dd}, J_{1}=6.4, J_{2}=2.0 \mathrm{~Hz}, 1 \mathrm{H}\right), 2.32(\mathrm{~s}, 3 \mathrm{H}), 1.95(\mathrm{~s}$, 
3H) $\cdot{ }^{13} \mathbf{C}$ NMR (100 MHz, $\left.\mathbf{C D C l}_{3}\right) \delta 174.31$ (q, $\left.J=35.1 \mathrm{~Hz}\right), 166.58,149.36,137.88,135.09$ (q, $J=4.9 \mathrm{~Hz}), 134.82,132.28,129.13,124.25,123.81(\mathrm{~d}, \mathrm{~J}=3.5 \mathrm{~Hz}), 120.59,120.42$ (d, $J=2.0$ Hz), 116.23 (q, $J=290.7 \mathrm{~Hz}), 110.12,100.96,56.06,44.84,26.13,20.08,17.68$. HRMS (ESI) calculated $[\mathrm{M}+\mathrm{H}]^{+}$for $\mathrm{C}_{24} \mathrm{H}_{19} \mathrm{O}_{3} \mathrm{NF}_{3}$ : 426.1312, found: 426.1313. FTIR (cm-1) 3442, 3024, 2924, 1763, 1672, 1516, 1452, 1390, 1146,1049, 906.

(7S,7aS,11aR)-7-(4-Chlorophenyl)-10-methyl-4-(2,2,2-trifluoroacetyl)-7,7adihydropyrano[3,4-c]pyrrolo[3,2,1-ij]quinolin-8(11aH)-one (3h)<smiles>CC1=CC2c3cccc4c(C(=O)C(F)(F)F)cn(c34)[C@H](c3ccc(Cl)cc3)[C@H]2C(=O)O1</smiles>

Following the general procedure, treatment of (E)-3-(4chlorophenyl)acrylaldehyde $\mathbf{2 h}(63.0 \mathrm{mg}, 0.375 \mathrm{mmol})$ and $(E)-4-(3-$ (2,2,2-trifluoroacetyl)-1H-indol-7-yl)but-3-en-2-one 1d (70.0 mg, 0.25 mmol) with triazolium salt 4 (15.0 $\mathrm{mg}, 0.037 \mathrm{mmol})$, oxidant 5 (153.0 $\mathrm{mg}, 0.375 \mathrm{mmol})$ and DABCO (28.0 $\mathrm{mg}, 0.25 \mathrm{mmol})$ in DMSO (2.0 $\mathrm{mL}$ ) and stirring the reaction mixture at $25{ }^{\circ} \mathrm{C}$ for $12 \mathrm{~h}$ followed by flash column chromatography (Pet. ether- EtOAc: 9:1) to afford (7S,7aS,11aR)-7-(4chlorophenyl)-10-methyl-4-(2,2,2-trifluoroacetyl)-7,7a-dihydropyrano[3,4-c]pyrrolo[3,2,1ij]quinolin-8(11aH)-one $\mathbf{3 h}$ as a white solid $(90.0 \mathrm{mg}, 81 \%$ yield).

$\boldsymbol{R}_{\boldsymbol{f}}($ Pet. ether $/$ EtOAc $=80 / 20): 0.45$; er $=93: 7,[\alpha]_{\mathrm{D}}{ }^{25}=+168.6\left(\mathrm{c} \mathrm{0.1}, \mathrm{CHCl}_{3}\right)$. HPLC (Chiralpak AD, 90:10 Hexane / IPA, $1.0 \mathrm{~mL} / \mathrm{min}$ ) Minor: $11.0 \mathrm{~min}$, Major: $12.6 \mathrm{~min}$.

${ }^{1}$ H NMR (400 MHz, CDCl$) \delta 8.24(\mathrm{~d}, J=8.0 \mathrm{~Hz}, 1 \mathrm{H}), 8.0(\mathrm{~s}, 1 \mathrm{H}), 7.37(\mathrm{t}, J=7.7 \mathrm{~Hz}, 1 \mathrm{H})$, 7.30-7.26 (m, 2H), $7.18(\mathrm{~d}, J=7.4 \mathrm{~Hz}, 1 \mathrm{H}), 6.76(\mathrm{~d}, J=8.1 \mathrm{~Hz}, 2 \mathrm{H}), 6.33(\mathrm{~s}, 1 \mathrm{H}), 5.66(\mathrm{~d}, J=$ $6.9 \mathrm{~Hz}, 1 \mathrm{H}), 3.69(\mathrm{t}, J=6.4 \mathrm{~Hz}, 1 \mathrm{H}), 3.37(\mathrm{~d}, J=6.3 \mathrm{~Hz}, 1 \mathrm{H}), 1.94(\mathrm{~s}, 3 \mathrm{H}) .{ }^{13} \mathbf{C}$ NMR (100 MHz, $\left.\mathbf{C D C l}_{3}\right) \delta 175.36(\mathrm{q}, J=35.5 \mathrm{~Hz}), 167.126,150.50,137.34,135.83$ (q, $\left.J=5.0 \mathrm{~Hz}\right), 134.94$, $133.17,129.73,126.80,124.98,124.84,124.82,121.77,121.56,121.16,116.95$ (q, $J=291.0$ $\mathrm{Hz}), 111.37,101.79,56.72,45.65,27.07$, 18.69. HRMS (ESI) calculated $[\mathrm{M}+\mathrm{H}]^{+}$for $\mathrm{C}_{23} \mathrm{H}_{16} \mathrm{O}_{3} \mathrm{~N}_{1} \mathrm{~F}_{3} \mathrm{Cl}:$ 446.0765, found: 446.0772. FTIR (cm $\left.{ }^{-1}\right)$ 3129, 2924, 1762, 1673, 1517, 1493, 1388, 1147, 1013, 906 . 
(7S,7aS,11aR)-7-(4-Bromophenyl)-10-methyl-4-(2,2,2-trifluoroacetyl)-7a,11a-dihydro7H,8H-pyrano[3,4-c]pyrrolo[3,2,1-ij]quinolin-8-one (3i)<smiles>CC1=CC2c3cccc4c(C(=O)C(F)(F)F)cn(c34)[C@@H](c3ccc(Br)cc3)[C@H]2C(=O)O1</smiles>

Following the general procedure, treatment of (E)-3-(4bromophenyl)acrylaldehyde $2 \mathbf{i}(79.1 \mathrm{mg}, 0.375 \mathrm{mmol})$ and $(E)-4-(3-$ (2,2,2-trifluoroacetyl)-1H-indol-7-yl)but-3-en-2-one 1d (70.0 mg, 0.25 mmol) with triazolium salt $4(15.0 \mathrm{mg}, 0.037 \mathrm{mmol})$, oxidant 5 (153.0 $\mathrm{mg}, 0.375 \mathrm{mmol})$ and DABCO $(28.0 \mathrm{mg}, 0.25 \mathrm{mmol})$ in DMSO $(2.0$ $\mathrm{mL}$ ) and stirring the reaction mixture at $25^{\circ} \mathrm{C}$ for $12 \mathrm{~h}$ followed by flash column chromatography (Pet. ether- EtOAc: 9:1) to afford (7S,7aS,11aR)-7-(4bromophenyl)-10-methyl-4-(2,2,2-trifluoroacetyl)-7a,11a-dihydro-7H,8H-pyrano[3,4c]pyrrolo[3,2,1-ij]quinolin-8-one $\mathbf{3 i}$ as a brown solid (107.0 $\mathrm{mg}, 87 \%$ yield). $\boldsymbol{R}_{\boldsymbol{f}}($ Pet. ether $/ \mathrm{EtOAc}=80 / 20): 0.46$; er $=93: 7,[\alpha]_{\mathrm{D}}{ }^{25}=159.40\left(\mathrm{c} 1.0, \mathrm{CHCl}_{3}\right)$. HPLC $($ Chiralpak AD, 90:10 Hexane / IPA, $1.0 \mathrm{~mL} / \mathrm{min}$ ) Minor: $11.3 \mathrm{~min}$, Major: $13.2 \mathrm{~min}$.

${ }^{1}$ H NMR (400 MHz, CDCl $) \delta 8.25(\mathrm{~d}, J=8.0 \mathrm{~Hz}, 1 \mathrm{H}), 7.98(\mathrm{~s}, 1 \mathrm{H}), 7.45(\mathrm{~d}, J=8.5 \mathrm{~Hz}, 2 \mathrm{H})$, $7.38(\mathrm{t}, J=7.7 \mathrm{~Hz}, 1 \mathrm{H}), 7.17(\mathrm{~d}, J=7.4 \mathrm{~Hz}, 1 \mathrm{H}), 6.70(\mathrm{~d}, J=8.4 \mathrm{~Hz}, 2 \mathrm{H}), 6.31(\mathrm{~s}, 1 \mathrm{H}), 5.66$ $(\mathrm{d}, J=7.0 \mathrm{~Hz}, 1 \mathrm{H}), 3.68(\mathrm{t}, J=6.6 \mathrm{~Hz}, 1 \mathrm{H}), 3.35\left(\mathrm{dd}, J_{1}=6.4 \mathrm{~Hz}, J_{2}=2.1 \mathrm{~Hz} 1 \mathrm{H}\right), 1.95(\mathrm{~s}, 3 \mathrm{H})$. ${ }^{13}$ C NMR (100 MHz, $\left.\mathbf{C D C l}_{3}\right) \delta 174.33$ (q, $\left.J=35.2 \mathrm{~Hz}\right), 166.23,149.49,136.86,134.38$, $132.14,126.08,123.98,123.80,121.98,120.78,120.55,120.16,115.93$ (q, $J=290.88 \mathrm{~Hz})$, 110.36, 100.77, 55.76, 44.57, 26.05, 17.70. HRMS (ESI) calculated $[\mathrm{M}+\mathrm{H}]^{+}$for $\mathrm{C}_{23} \mathrm{H}_{16} \mathrm{BrF}_{3} \mathrm{NO}_{3}$ : 490.0260, found: 490.0267. FTIR (cm $\left.{ }^{-1}\right)$ 3437, 3130, 3024, 2924, 1762, 1672 , $1517,1452,1146,1048,905,833$.

(7S,7aS,11aR)-10-Methyl-4-(2,2,2-trifluoroacetyl)-7-(4-(trifluoromethyl)phenyl)-7,7adihydropyrano[3,4-c]pyrrolo[3,2,1-ij]quinolin-8(11aH)-one (3j)<smiles>CC1=C[C@H]2c3cccc4c(C(=O)C(F)(F)F)cn(c34)[C@H](c3ccc(C(F)(F)F)cc3)[C@H]2C(=O)O1</smiles>

Following the general procedure, treatment of (E)-3-(4(trifluoromethyl)phenyl)acrylaldehyde $\mathbf{2 j}$ ( $75.0 \mathrm{mg}, 0.375 \mathrm{mmol}$ ) and (E)-4-(3-(2,2,2-trifluoroacetyl)-1H-indol-7-yl)but-3-en-2-one 1d (70.0 $\mathrm{mg}, 0.25 \mathrm{mmol})$ with triazolium salt $4(15.0 \mathrm{mg}, 0.037 \mathrm{mmol})$, oxidant 5 (153.0 $\mathrm{mg}, 0.375 \mathrm{mmol})$ and DABCO $(28.0 \mathrm{mg}, 0.25$ mmol) in DMSO $(2.0 \mathrm{~mL})$ and stirring the reaction mixture at $25{ }^{\circ} \mathrm{C}$ for $12 \mathrm{~h}$ followed by flash column chromatography (Pet. ether- EtOAc: 9:1) to afford 
(7S,7aS,11aR)-10-Methyl-4-(2,2,2-trifluoroacetyl)-7-(4-(trifluoromethyl)phenyl)-7,7a-

dihydropyrano[3,4-c]pyrrolo[3,2,1-ij]quinolin-8(11aH)-one $\mathbf{3 j}$ as a white solid $(108.0 \mathrm{mg}, 90 \%$ yield).

$\boldsymbol{R}_{\boldsymbol{f}}($ Pet. ether $/$ EtOAc $=80 / 20): 0.45$; er $=92: 8,[\alpha]_{\mathrm{D}}{ }^{25}=+130.7\left(\mathrm{c} 0.1, \mathrm{CHCl}_{3}\right)$. HPLC $($ Chiralpak IA, 90:10 Hexane / IPA, $1.0 \mathrm{~mL} / \mathrm{min}$ ) Minor: $8.7 \mathrm{~min}$, Major: $11.6 \mathrm{~min}$.

${ }^{1}$ H NMR (400 MHz, CDCl $) \delta 8.25(\mathrm{~d}, J=8.0 \mathrm{~Hz}, 1 \mathrm{H}), 8.02(\mathrm{~d}, J=1.4 \mathrm{~Hz}, 1 \mathrm{H}), 7.59$ (d, $J=$ $8.2 \mathrm{~Hz}, 2 \mathrm{H}), 7.39$ (t, $J=7.7 \mathrm{~Hz}, 1 \mathrm{H}), 7.20$ (d, $J=7.4 \mathrm{~Hz}, 1 \mathrm{H}), 6.96$ (d, $J=8.1 \mathrm{~Hz}, 2 \mathrm{H}), 6.44$ (s, $1 \mathrm{H}), 5.68(\mathrm{~d}, J=7.0 \mathrm{~Hz}, 1 \mathrm{H}), 3.67(\mathrm{t}, J=6.6 \mathrm{~Hz}, 1 \mathrm{H}), 3.43\left(\mathrm{dd}, J_{l}=6.4 \mathrm{~Hz}, J_{2}=1.9 \mathrm{~Hz}, 1 \mathrm{H}\right)$, 1.95 (s, 3H). ${ }^{13} \mathbf{C}$ NMR (100 MHz, CDCl 3$) \delta 175.42$ (q, $J=34.9$ Hz), 167.14, 150.62, 142.86, $135.76(\mathrm{q}, J=5.0 \mathrm{~Hz}), 133.16,131.29$ (q, $J=33.1 \mathrm{~Hz}), 126.59$ (q, $J=3.8 \mathrm{~Hz}), 125.95,125.09$, $124.85,122.36,121.89,121.66,121.11,116.95$ (q, $J=292.0 \mathrm{~Hz}), 111.53,101.70,56.95,45.57$, 27.07, 18.68. HRMS (ESI) calculated $[\mathrm{M}+\mathrm{H}]^{+}$for $\mathrm{C}_{24} \mathrm{H}_{16} \mathrm{O}_{3} \mathrm{~N}_{1} \mathrm{~F}_{6}: 480.1029$, found: 480.1039 . FTIR (cm $\left.{ }^{-1}\right)$ 3445, 2926, 1763, 1674, 1518, 1452, 1390, 1326, 1147, 1015, 906.

Methyl 4-((7S,7aS,11aR)-10-methyl-8-oxo-4-(2,2,2-trifluoroacetyl)-7a,11a-dihydro-7H, $8 H$ pyrano[3,4-c]pyrrolo[3,2,1-ij]quinolin-7-yl)benzoate (3k)

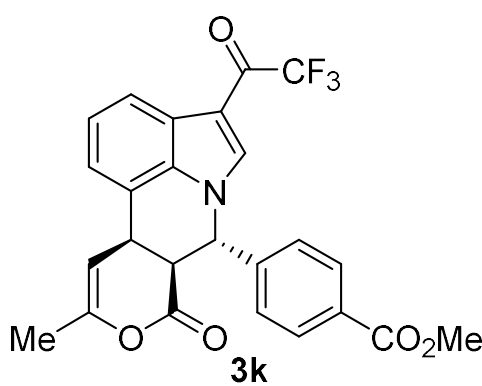

Following the general procedure, treatment of methyl $(E)-4-(3-$ oxoprop-1-en-1-yl)benzoate $2 \mathbf{k}(95 \mathrm{mg}, 0.5 \mathrm{mmol})$ and $(E)-4-(3-$ (2,2,2-trifluoroacetyl)-1H-indol-7-yl)but-3-en-2-one 1 d $\quad(70.0$ $\mathrm{mg}, 0.25 \mathrm{mmol})$ with triazolium salt $4(15.0 \mathrm{mg}, 0.037 \mathrm{mmol})$, oxidant 5 (153.0 $\mathrm{mg}, 0.375 \mathrm{mmol})$ and DABCO (28.0 mg, $0.25 \mathrm{mmol})$ in DMSO $(2.0 \mathrm{~mL})$ and stirring the reaction mixture at $25{ }^{\circ} \mathrm{C}$ for $12 \mathrm{~h}$ followed by flash column chromatography (Pet. ether- EtOAc: 9:1) to afford methyl 4-(7S,7aS,11aR)-10-methyl-7-phenyl-4-(2,2,2-trifluoroacetyl)-7,7a-dihydropyrano[3,4c]pyrrolo[3,2,1-ij]quinolin-8(11aH)-one 3k as a black solid (84.5 mg, 72\% yield).

$\boldsymbol{R}_{\boldsymbol{f}}($ Pet. ether $/$ EtOAc $=80 / 20): 0.42 ;$ er $=87: 13,[\alpha]_{\mathrm{D}}{ }^{25}=121.28\left(\mathrm{c} 1.0, \mathrm{CHCl}_{3}\right)$. HPLC (Chiralpak AD, 90:10 Hexane / IPA, 1.0 mL/min) Minor: 18.4 min, Major: $20.4 \mathrm{~min}$.

${ }^{1}$ H NMR (400 MHz, CDCl $\left.{ }_{3}\right) \delta 8.24(\mathrm{~d}, J=8.0 \mathrm{~Hz}, 1 \mathrm{H}), 7.99(\mathrm{t}, J=7.97 \mathrm{~Hz}, 3 \mathrm{H}), 7.38(\mathrm{t}, J=$ $7.6 \mathrm{~Hz}, 1 \mathrm{H}), 7.17(\mathrm{~d}, J=7.2 \mathrm{~Hz}, 1 \mathrm{H}), 6.89(\mathrm{~d}, J=8.1 \mathrm{~Hz}, 2 \mathrm{H}), 6.41(\mathrm{~s}, 1 \mathrm{H}), 5.66(\mathrm{~d}, J=6.7 \mathrm{~Hz}$, 1H), 3.89 (s, 3H), 3.65 (s, 1H), 3.42 (d, $J=5.9 \mathrm{~Hz}, 1 \mathrm{H}), 1.94(\mathrm{~s}, 3 \mathrm{H}) .{ }^{13} \mathbf{C}$ NMR (100 MHz, $\left.\mathbf{C D C l}_{3}\right) \delta 175.39$ (q, $\left.J=35.2 \mathrm{~Hz}\right), 167.20,166.23,150.54,143.66,135.85$ (q, $\left.J=5.0 \mathrm{~Hz}\right)$, 
$133.18,130.86,130.78,125.51,125.01,124.84,121.79,121.63,121.12,116.94$ (q, $J=291 \mathrm{~Hz}$ ), 111.44, 101.76, 57.07, 52.46, 45.57, 27.10, 18.72. HRMS (ESI) calculated $[\mathrm{M}+\mathrm{H}]^{+}$for $\mathrm{C}_{23} \mathrm{H}_{17} \mathrm{O}_{3} \mathrm{~N}_{1} \mathrm{~F}_{3}:$ 412.1155, found: 412.1161. FTIR (cm $\left.{ }^{-1}\right)$ 3441, 3023, 2954, 1763, 1722, 1672 , $1518,1390,1285,1146,906$.

(7S,7aS,11aR)-7-(3-Bromophenyl)-10-methyl-4-(2,2,2-trifluoroacetyl)-7,7adihydropyrano[3,4-c]pyrrolo[3,2,1-ij]quinolin-8(11aH)-one (3I)

Following the general procedure, treatment of $(E)-3-(3-$
bromophenyl)acrylaldehyde $21(79.0 \mathrm{mg}, 0.375 \mathrm{mmol})$ and $(E)-4-(3-(2,2,2-$ chromatography (Pet. ether- EtOAc: 9:1) to afford (7S,7aS,11aR)-7-(3-bromophenyl)-10methyl-4-(2,2,2-trifluoroacetyl)-7,7a-dihydropyrano[3,4-c]pyrrolo[3,2,1-ij]quinolin-8(11aH)-one $3 \mathrm{I}$ as a white solid (112.0 $\mathrm{mg}, 91 \%$ yield).

$\boldsymbol{R}_{\boldsymbol{f}}($ Pet. ether $/$ EtOAc $=80 / 20): 0.45$; er $=95: 5,[\alpha]_{\mathrm{D}}{ }^{25}=+152.7\left(\mathrm{c} \mathrm{0.1}, \mathrm{CHCl}_{3}\right)$. HPLC $($ Chiralpak IA, 90:10 Hexane / IPA, $1.0 \mathrm{~mL} / \mathrm{min}$ ) Minor: $9.3 \mathrm{~min}$, Major: $10.1 \mathrm{~min}$.

${ }^{1}$ H NMR (400 MHz, CDCl 3$) \delta 8.24(\mathrm{~d}, J=7.9 \mathrm{~Hz}, 1 \mathrm{H}), 8.01(\mathrm{~s}, 1 \mathrm{H}), 7.46(\mathrm{~d}, J=7.9 \mathrm{~Hz}, 1 \mathrm{H})$, $7.38(\mathrm{t}, J=7.7 \mathrm{~Hz}, 1 \mathrm{H}), 7.19-7.15(\mathrm{~m}, 2 \mathrm{H}), 7.10(\mathrm{~s}, 1 \mathrm{H}), 6.64(\mathrm{~d}, J=7.7 \mathrm{~Hz}, 1 \mathrm{H}), 6.33(\mathrm{~s}, 1 \mathrm{H})$, $5.67(\mathrm{~d}, J=7.0 \mathrm{~Hz}, 1 \mathrm{H}), 3.70(\mathrm{t}, J=6.4 \mathrm{~Hz}, 1 \mathrm{H}), 3.40(\mathrm{~d}, J=6.3 \mathrm{~Hz}, 1 \mathrm{H}), 1.94(\mathrm{~s}, 3 \mathrm{H}) .{ }^{13} \mathrm{C}$ NMR (100 MHz, CDCl $) \delta 175.37$ (q, $J=35.3 \mathrm{~Hz}$ ), 167.19, 150.53, 141.17, 135.80 133.12, $132.15,131.12,128.53,124.98,124.80,123.99,123.67,121.81,121.60,121.10,116.94$ (q, $J$ $=291.0 \mathrm{~Hz}), 111.45,101.77,56.68,45.66,27.04,18.70$. HRMS (ESI) calculated $[\mathrm{M}+\mathrm{H}]^{+}$for $\mathrm{C}_{23} \mathrm{H}_{16} \mathrm{O}_{3} \mathrm{~N}_{1} \mathrm{~F}_{3} \mathrm{Br}$ : 490.0260, found: 490.0265. FTIR (cm $\left.{ }^{-1}\right)$ 3440, 2924, 1762, 1672, 1517, 1389, $1147,1048,1015,906$.

\section{(7S,7aS,11aR)-7-(2-Methoxyphenyl)-10-methyl-4-(2,2,2-trifluoroacetyl)-7a,11a-dihydro-}<smiles>COc1ccccc1[C@@H]1[C@H]2C(=O)OC(C)=C[C@H]2c2cccc3c(C(=O)C(F)(F)F)cn1c23</smiles>

\section{H,8H-pyrano[3,4-c]pyrrolo[3,2,1-ij]quinolin-8-one (3m)}

Following the general procedure, treatment of 2-methoxy cinnamaldehyde $\mathbf{2 m}$ (121.6 mg, $0.75 \mathrm{mmol})$ and $(E)-4-(3-(2,2,2-$ 
trifluoroacetyl)-1H-indol-7-yl)but-3-en-2-one $1 \mathrm{~d}(140.6 \mathrm{mg}, 0.5 \mathrm{mmol})$ with triazolium salt 4 (27.6 mg, $0.075 \mathrm{mmol})$, oxidant $5(306.0 \mathrm{mg}, 0.75 \mathrm{mmol})$ and DABCO (56.0 mg, $0.5 \mathrm{mmol})$ in DMSO $(4.0 \mathrm{~mL})$ and stirring the reaction mixture at $25^{\circ} \mathrm{C}$ for $12 \mathrm{~h}$ followed by flash column chromatography (Pet. ether- EtOAc: 9:1) to afford (7S,7aS,11aR)-7-(2-methoxyphenyl)-10methyl-4-(2,2,2-trifluoroacetyl)-7a,11a-dihydro-7H,8H-pyrano[3,4-c]pyrrolo[3,2,1-ij]quinolin-8one $\mathbf{3 m}$ as a yellow solid (200.0 $\mathrm{mg}, 90 \%$ yield).

$\boldsymbol{R}_{\boldsymbol{f}}($ Pet. ether $/$ EtOAc $=80 / 20): 0.42$; er $=98: 2,[\alpha]_{\mathrm{D}}{ }^{25}=181.52\left(\mathrm{c} 1.0, \mathrm{CHCl}_{3}\right)$. HPLC $($ Chiralpak AD, 90:10 Hexane / IPA, $1.0 \mathrm{~mL} / \mathrm{min}$ ) Minor: $5.5 \mathrm{~min}$, Major: $7.8 \mathrm{~min}$.

${ }^{1}$ H NMR (400 MHz, CDCl $) \delta 8.24(\mathrm{~d}, J=8.0 \mathrm{~Hz}, 1 \mathrm{H}), 8.02(\mathrm{~s}, 1 \mathrm{H}), 7.36(\mathrm{t}, J=7.7 \mathrm{~Hz}, 1 \mathrm{H})$, $7.32-7.28(\mathrm{~m}, 1 \mathrm{H}), 7.16(\mathrm{~d}, J=7.3 \mathrm{~Hz}, 1 \mathrm{H}), 6.99(\mathrm{~d}, J=8.2 \mathrm{~Hz}, 1 \mathrm{H}), 6.75(\mathrm{t}, J=7.5 \mathrm{~Hz}, 1 \mathrm{H})$, $6.67(\mathrm{~s}, 1 \mathrm{H}), 6.01(\mathrm{~d}, J=7.5 \mathrm{~Hz}, 1 \mathrm{H}), 5.65(\mathrm{~d}, J=6.9 \mathrm{~Hz}, 1 \mathrm{H}), 3.94(\mathrm{~s}, 3 \mathrm{H}) 3.65-3.58(\mathrm{~m}, 2 \mathrm{H})$ 1.95 (s, 3H). ${ }^{13} \mathbf{C}$ NMR (100 MHz, CDCl $) \delta 174.35(\mathrm{q}, J=35.1 \mathrm{~Hz}), 166.85,154.26,149.42$, $135.17(\mathrm{q}, J=4.9 \mathrm{~Hz}), 132.75,129.03,127.44,125.79,125.01,123.82,120.60,120.50,120.41$, $120.26,116.05(\mathrm{q}, J=290.9 \mathrm{~Hz}), 110.14,109.85,100.98,54.71,51$

.97, 41.83, 26.50, 17.76. HRMS (ESI) calculated $[\mathrm{M}+\mathrm{H}]^{+}$for $\mathrm{C}_{24} \mathrm{H}_{19} \mathrm{~F}_{3} \mathrm{NO}_{4}$ : 442.1261, found: 442.1265. FTIR (cm-1) 3130, 3022, 2961, 1763, 1672, 1597, 1518, 1458, 1391 , 1145, 906.

\section{(7S,7aS,11aR)-10-Methyl-7-(o-tolyl)-4-(2,2,2-trifluoroacetyl)-7,7a-dihydropyrano[3,4- c]pyrrolo[3,2,1-ij]quinolin-8(11aH)-one (3n)}

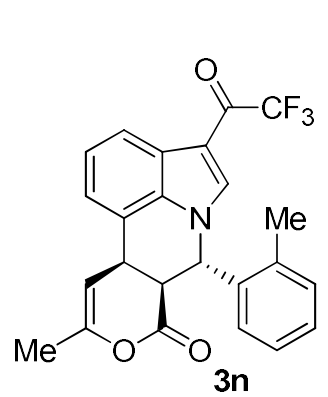

Following the general procedure, treatment of $(E)-3-(0-$ tolyl)acrylaldehyde $2 \mathrm{n}(55.0 \mathrm{mg}, 0.375 \mathrm{mmol})$ and $(E)-4-(3-(2,2,2-$ trifluoroacetyl)-1 $H$-indol-7-yl)but-3-en-2-one 1 d $(70.0 \mathrm{mg}, 0.25 \mathrm{mmol})$ with triazolium salt $4(15.0 \mathrm{mg}, 0.037 \mathrm{mmol})$, oxidant $5(153.0 \mathrm{mg}$, $0.375 \mathrm{mmol})$ and DABCO $(28.0 \mathrm{mg}, 0.25 \mathrm{mmol})$ in DMSO $(2.0 \mathrm{~mL})$ and stirring the reaction mixture at $25{ }^{\circ} \mathrm{C}$ for $12 \mathrm{~h}$ followed by flash column chromatography (Pet. ether- EtOAc: 9:1) to afford (7S,7aS,11aR)-10-methyl-7-(o-tolyl)4-(2,2,2-trifluoroacetyl)-7,7a-dihydropyrano[3,4-c]pyrrolo[3,2,1-ij]quinolin-8(11a $H)$-one $\mathbf{3 n}$ as a white solid (98.0 mg, 92\% yield).

$\boldsymbol{R}_{\boldsymbol{f}}($ Pet. ether $/ \mathrm{EtOAc}=80 / 20): 0.50 ;$ er $=96: 4,[\alpha]_{\mathrm{D}}{ }^{25}=+263.2\left(\mathrm{c} 0.1, \mathrm{CHCl}_{3}\right)$. HPLC $($ Chiralpak AD, 90:10 Hexane / IPA, $1.0 \mathrm{~mL} / \mathrm{min}$ ) Minor: $5.0 \mathrm{~min}$, Major: $6.0 \mathrm{~min}$. 
${ }^{1}$ H NMR (400 MHz, CDCl $) \delta 8.26(\mathrm{~d}, J=8.0 \mathrm{~Hz}, 1 \mathrm{H}), 7.98(\mathrm{~s}, 1 \mathrm{H}), 7.39$ (t, $\left.J=7.7 \mathrm{~Hz}, 1 \mathrm{H}\right)$, 7.30-7.19 (m, 3H), 7.01 (t, $J=7.5 \mathrm{~Hz}, 1 \mathrm{H}), 6.56(\mathrm{~s}, 1 \mathrm{H}), 6.14(\mathrm{~d}, J=7.7 \mathrm{~Hz}, 1 \mathrm{H}), 5.68$ (d, $J=$ $7.0 \mathrm{~Hz}, 1 \mathrm{H}), 3.73$ (t, $J=6.5 \mathrm{~Hz}, 1 \mathrm{H}), 3.29$ (d, $J=6.3 \mathrm{~Hz}, 1 \mathrm{H}), 2.52(\mathrm{~s}, 3 \mathrm{H}), 1.96(\mathrm{~s}, 3 \mathrm{H}) .{ }^{13} \mathbf{C}$ NMR (100 MHz, $\left.\mathbf{C D C l}_{3}\right) \delta 175.37$ (q, $\left.J=35.4 \mathrm{~Hz}\right), 167.57,150.43,136.94,136.06$ (q, $J=5.1$ $\mathrm{Hz}), 133.83,133.78,131.57,128.83,127.15,125.14,124.83,124.72,121.54,121.23,116.98$ (q, $J=291.0 \mathrm{~Hz}), 111.27,102.00,54.53,43.55,27.10,19.00,18.73$. HRMS (ESI) calculated $[\mathrm{M}+\mathrm{H}]^{+}$for $\mathrm{C}_{24} \mathrm{H}_{19} \mathrm{O}_{3} \mathrm{~N}_{1} \mathrm{~F}_{3}$ : 426.1312, found: 426.1317. FTIR (cm $\left.\mathbf{c m}^{-1}\right)$ 3424, 1759, 1671, 1517 , $1454,1397,1346,1145,907$.

\section{(7S,7aS,11aR)-7-(2-Fluorophenyl)-10-methyl-4-(2,2,2-trifluoroacetyl)-7,7a-}

\section{dihydropyrano[3,4-c]pyrrolo[3,2,1-ij]quinolin-8(11aH)-one (3o)} Following the general procedure, treatment of $(E)-3-(2-$
fluorophenyl)acrylaldehyde $20(56.0 \mathrm{mg}, 0.375 \mathrm{mmol})$ and $(E)-4-(3-(2,2,2-$
trifluoroacetyl)-1H-indol-7-yl but-3-en-2-one 1d $(70.0 \mathrm{mg}, 0.25 \mathrm{mmol})$ chromatography (Pet. ether- EtOAc: 9:1) to afford (7S,7aS,11aR)-7-(2-fluorophenyl)-10-methyl4-(2,2,2-trifluoroacetyl)-7,7a-dihydropyrano[3,4-c]pyrrolo[3,2,1-ij]quinolin-8 (11a $H)$-one 3o as a white solid (79.0 $\mathrm{mg}, 74 \%$ yield).

$\boldsymbol{R}_{\boldsymbol{f}}($ Pet. ether $/$ EtOAc $=80 / 20): 0.40 ;$ er $=93: 7,[\alpha]_{\mathrm{D}}{ }^{25}=+130.2\left(\mathrm{c} 0.1, \mathrm{CHCl}_{3}\right)$. HPLC $($ Chiralpak AD, 90:10 Hexane / IPA, $1.0 \mathrm{~mL} / \mathrm{min}$ ) Minor: $6.7 \mathrm{~min}$, Major: $8.0 \mathrm{~min}$.

${ }^{1}$ H NMR (400 MHz, CDCl$) \delta 8.25(\mathrm{~d}, J=8.0 \mathrm{~Hz}, 1 \mathrm{H}), 8.01(\mathrm{~s}, 1 \mathrm{H}), 7.40-7.31(\mathrm{~m}, 2 \mathrm{H}), 7.20$ $7.15(\mathrm{~m}, 2 \mathrm{H}), 6.97$ (t, $J=7.5 \mathrm{~Hz}, 1 \mathrm{H}), 6.65(\mathrm{~s}, 1 \mathrm{H}), 6.12$ (t, $J=7.5 \mathrm{~Hz}, 1 \mathrm{H}), 5.67$ (d, $J=6.8 \mathrm{~Hz}$, 1H), $3.67(\mathrm{t}, J=6.4 \mathrm{~Hz}, 1 \mathrm{H}), 3.51-3.49(\mathrm{~m}, 1 \mathrm{H}), 1.95(\mathrm{~s}, 3 \mathrm{H}) .{ }^{13} \mathbf{C} \mathbf{N M R}\left(\mathbf{1 0 0} \mathbf{M H z}, \mathbf{C D C l}_{3}\right) \delta$ 175.40 (q, $J=35.2 \mathrm{~Hz}), 167.12,158.83(\mathrm{~d}, J=247.0 \mathrm{~Hz}), 150.61,135.83$ (q, $J=4.9 \mathrm{~Hz})$, $133.43,130.78(\mathrm{~d}, J=8.1 \mathrm{~Hz}), 126.84(\mathrm{~d}, J=3.0 \mathrm{~Hz}), 126.10,125.97,125.25$ (d, $J=3.0 \mathrm{~Hz})$, 125.01, 124.84, 121.75, 121.62, 121.19, 116.97 (q, $J=291.0 \mathrm{~Hz}$ ), 116.34, 116.13, 111.42, $101.74,52.13(\mathrm{~d}, J=3.2 \mathrm{~Hz}), 43.69,27.40,18.76$. HRMS (ESI) calculated $[\mathrm{M}+\mathrm{H}]^{+}$for $\mathrm{C}_{23} \mathrm{H}_{16} \mathrm{O}_{3} \mathrm{~N}_{1} \mathrm{~F}_{4}$ : 430.1061, found: 430.1065. FTIR (cm $\left.{ }^{-1}\right)$ 2925, 2125, 1763, 1673, 1519, 1454, 1394, 1147, 1048, 907. 
(7S,7aS,11aR)-7-(2-Chlorophenyl)-10-methyl-4-(2,2,2-trifluoroacetyl)-7,7adihydropyrano[3,4-c]pyrrolo[3,2,1-ij]quinolin-8(11aH)-one (3p)<smiles>CC1=CC2c3cccc4c(C(=O)C(F)(F)F)cn(c34)[C@H](c3ccccc3Cl)[C@H]2C(=O)O1</smiles>

Following the general procedure, treatment of $(E)-3-(2-$ chlorophenyl)acrylaldehyde $\mathbf{2 p}(63.0 \mathrm{mg}, 0.375 \mathrm{mmol})$ and $(E)-4-(3-$ (2,2,2-trifluoroacetyl)-1H-indol-7-yl)but-3-en-2-one $\mathbf{1 d ~ ( 7 0 . 0 ~ m g , ~} 0.25$ mmol) with triazolium salt $4(15.0 \mathrm{mg}, 0.037 \mathrm{mmol})$, oxidant $5(153.0 \mathrm{mg}$, $0.375 \mathrm{mmol})$ and DABCO (28.0 $\mathrm{mg}, 0.25 \mathrm{mmol})$ in DMSO $(2.0 \mathrm{~mL})$ and stirring the reaction mixture at $25{ }^{\circ} \mathrm{C}$ for $12 \mathrm{~h}$ followed by flash column chromatography (Pet. ether- EtOAc: 9:1) to afford (7S,7aS,11aR)-7-(2-chlorophenyl)-10methyl-4-(2,2,2-trifluoroacetyl)-7,7a-dihydropyrano[3,4-c]pyrrolo[3,2,1-ij]quinolin-8(11a $H)$-one $3 \mathbf{p}$ as a white solid (106.0 $\mathrm{mg}$, 95\% yield).

$\boldsymbol{R}_{\boldsymbol{f}}($ Pet. ether $/$ EtOAc $=80 / 20): 0.50 ;$ er $=94: 6,[\alpha]_{\mathrm{D}}{ }^{25}=+213.4\left(\mathrm{c} 0.1, \mathrm{CHCl}_{3}\right)$. HPLC $($ Chiralpak AD, 90:10 Hexane / IPA, $1.0 \mathrm{~mL} / \mathrm{min}$ ) Minor: $5.2 \mathrm{~min}$, Major: $6.2 \mathrm{~min}$.

${ }^{1}$ H NMR (400 MHz, CDCl $) \delta 8.25(\mathrm{~d}, J=8.0 \mathrm{~Hz}, 1 \mathrm{H}), 8.01(\mathrm{~s}, 1 \mathrm{H}), 7.48(\mathrm{~d}, J=7.9 \mathrm{~Hz}, 1 \mathrm{H})$, $7.38(\mathrm{t}, J=7.7 \mathrm{~Hz}, 1 \mathrm{H}), 7.31-7.26(\mathrm{~m}, 1 \mathrm{H}), 7.19(\mathrm{~d}, J=7.3 \mathrm{~Hz}, 1 \mathrm{H}), 7.08(\mathrm{t}, J=7.4 \mathrm{~Hz}, 1 \mathrm{H})$, $6.73(\mathrm{~s}, 1 \mathrm{H}), 6.76(\mathrm{~d}, J=8.1 \mathrm{~Hz}, 2 \mathrm{H}), 6.33(\mathrm{~s}, 1 \mathrm{H}), 6.14(\mathrm{~d}, J=7.7 \mathrm{~Hz}, 1 \mathrm{H}), 5.68(\mathrm{~d}, J=6.8 \mathrm{~Hz}$, 1H), $3.64(\mathrm{t}, J=6.3 \mathrm{~Hz}, 1 \mathrm{H}), 3.57(\mathrm{~d}, J=6.4 \mathrm{~Hz}, 1 \mathrm{H}), 1.95(\mathrm{~s}, 3 \mathrm{H}) .{ }^{13} \mathbf{C}$ NMR (100 MHz, $\left.\mathbf{C D C l}_{3}\right) \delta 175.36(\mathrm{q}, J=35.4 \mathrm{~Hz}), 167.17,150.59,136.12,135.88,133.53,131.26,130.56$, $130.25,127.96,126.83,124.98,124.71,121.72,121.53,121.24,116.92$ (q, $J=291.0 \mathrm{~Hz}$ ), $111.47,101.68,77.48,77.16,76.84,54.95,54.91,42.84,27.20,18.72,18.70$. HRMS (ESI) calculated $[\mathrm{M}+\mathrm{H}]^{+}$for $\mathrm{C}_{23} \mathrm{H}_{16} \mathrm{O}_{3} \mathrm{~N}_{1} \mathrm{~F}_{3} \mathrm{Cl}$ : 446.0765, found: 446.0771. FTIR (cm $\left.{ }^{-1}\right)$ 3440, 2959, $1762,1675,1518,1447,1391,1147,1042,907$.

\section{(7S,7aS,11aR)-7-(3,4-Dichlorophenyl)-10-methyl-4-(2,2,2-trifluoroacetyl)-7,7a-}

\section{dihydropyrano[3,4-c]pyrrolo[3,2,1-ij]quinolin-8(11aH)-one (3q)}<smiles>COC(=O)C1OC(C)=C[C@@H]2C1c1cccc3c(C(=O)C(F)(F)F)cn(c13)[C@H]2c1ccc(Cl)c(Cl)c1</smiles>

Following the general procedure, treatment of $(E)-3-(3,4-$ dichlorophenyl)acrylaldehyde $\mathbf{2 q}(75.0 \mathrm{mg}, 0.375 \mathrm{mmol})$ and $(E)-4-(3-$ (2,2,2-trifluoroacetyl)- $1 H$-indol-7-yl)but-3-en-2-one 1d (70.0 mg, 0.25 mmol) with triazolium salt 4 (15.0 mg, $0.037 \mathrm{mmol})$, oxidant 5 (153.0 $\mathrm{mg}, 0.375 \mathrm{mmol})$ and DABCO $(28.0 \mathrm{mg}, 0.25 \mathrm{mmol})$ in DMSO $(2.0$ $\mathrm{mL}$ ) and stirring the reaction mixture at $25{ }^{\circ} \mathrm{C}$ for $12 \mathrm{~h}$ followed by 
flash column chromatography (Pet. ether- EtOAc: 9:1) to afford (7S,7aS,11aR)-7-(3,4dichlorophenyl)-10-methyl-4-(2,2,2-trifluoroacetyl)-7,7a-dihydropyrano[3,4-c]pyrrolo[3,2,1ij]quinolin-8(11a $H)$-one $\mathbf{3 q}$ as a white solid (100.0 $\mathrm{mg}, 83 \%$ yield).

$\boldsymbol{R}_{\boldsymbol{f}}($ Pet. ether $/$ EtOAc $=80 / 20): 0.50 ;$ er $=93: 7,[\alpha]_{\mathrm{D}}{ }^{25}=+149.5\left(\mathrm{c} 0.1, \mathrm{CHCl}_{3}\right)$. HPLC $($ Chiralpak IB, 90:10 Hexane / IPA, $1.0 \mathrm{~mL} / \mathrm{min}$ ) Minor: $12.2 \mathrm{~min}$, Major: $13.8 \mathrm{~min}$.

${ }^{1}$ H NMR (400 MHz, CDCl $) \delta 8.24(\mathrm{~d}, J=8.0 \mathrm{~Hz}, 1 \mathrm{H}), 7.99$ (s, 1H), 7.40-7.36 (m, 2H), 7.19 (d, $J=7.2 \mathrm{~Hz}, 1 \mathrm{H}), 7.01(\mathrm{~s}, 1 \mathrm{H}), 6.58(\mathrm{~d}, J=8.3 \mathrm{~Hz}, 1 \mathrm{H}), 6.32(\mathrm{~s}, 1 \mathrm{H}), 5.67(\mathrm{~d}, J=7.0 \mathrm{~Hz}, 1 \mathrm{H})$, $3.69(\mathrm{t}, J=6.3 \mathrm{~Hz}, 1 \mathrm{H}), 3.37(\mathrm{~d}, J=6.3 \mathrm{~Hz}, 1 \mathrm{H}), 1.94(\mathrm{~s}, 3 \mathrm{H}) .{ }^{13} \mathbf{C} \mathbf{~ N M R}\left(\mathbf{1 0 0} \mathbf{M H z}, \mathbf{C D C l}_{\mathbf{3}}\right) \delta$ $175.40(\mathrm{q}, J=35.2 \mathrm{~Hz}), 167.0,150.61,139.09,135.64(\mathrm{q}, J=4.9 \mathrm{~Hz}), 133.96,133.36,133.0$, $131.56,127.52,125.09,124.84,124.75,121.92,121.68,120.96,116.91(\mathrm{q}, J=291.0 \mathrm{~Hz})$, 111.58, 101.68, 56.36, 45.54, 27.07, 18.69. HRMS (ESI) calculated $[\mathrm{M}+\mathrm{H}]^{+}$for $\mathrm{C}_{23} \mathrm{H}_{15} \mathrm{O}_{3} \mathrm{~N}_{1} \mathrm{~F}_{3} \mathrm{Cl}_{2}$ : 480.0376, found: 480.0380. FTIR (cm $\left.{ }^{-1}\right)$ 3430, 2924, 1764, 1673, 1518, $1146,1049,907$.

(7S,7aS,11aR)-7-(Benzo[d][1,3]dioxol-5-yl)-10-methyl-4-(2,2,2-trifluoroacetyl)-7,7adihydropyrano[3,4-c]pyrrolo[3,2,1-ij]quinolin-8(11aH)-one (3r)

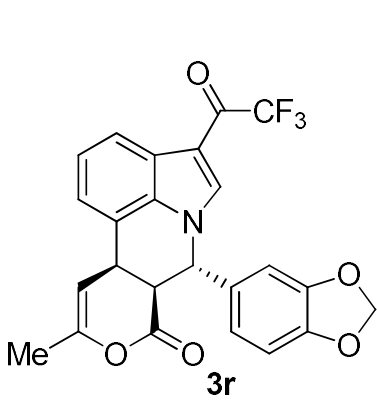

Following the general procedure, treatment of (E)-3(benzo[d][1,3]dioxol-4-yl)acrylaldehyde 2 r $(66.0 \mathrm{mg}, 0.375 \mathrm{mmol})$ and (E)-4-(3-(2,2,2-trifluoroacetyl)-1H-indol-7-yl)but-3-en-2-one 1d (70.0 $\mathrm{mg}, 0.25 \mathrm{mmol})$ with triazolium salt $4(15.0 \mathrm{mg}, 0.037 \mathrm{mmol})$, oxidant $5(153.0 \mathrm{mg}, 0.375 \mathrm{mmol})$ and DABCO $(28.0 \mathrm{mg}, 0.25 \mathrm{mmol})$ in DMSO $(2.0 \mathrm{~mL})$ and stirring the reaction mixture at $25{ }^{\circ} \mathrm{C}$ for $12 \mathrm{~h}$ followed by flash column chromatography (Pet. ether- EtOAc: 9:1) to afford (7S,7aS,11aR)-7(benzo[d][1,3]dioxol-5-yl)-10-methyl-4-(2,2,2-trifluoroacetyl)-7,7a-dihydropyrano[3,4c]pyrrolo[3,2,1-ij]quinolin-8(11aH)-one $3 \mathrm{r}$ as a white solid (94.0 $\mathrm{mg}, 82 \%$ yield).

$\boldsymbol{R}_{\boldsymbol{f}}($ Pet. ether $/$ EtOAc $=80 / 20): 0.40 ;$ er $=97: 3,[\alpha]_{\mathrm{D}}^{25}=+186.92\left(\mathrm{c} 0.1, \mathrm{CHCl}_{3}\right)$. HPLC (Chiralpak IA, 90:10 Hexane / IPA, 1.0 mL/min) Minor: 16.9 min, Major: $18.8 \mathrm{~min}$.

${ }^{1}$ H NMR (400 MHz, CDCl $\left.\mathbf{l}_{3}\right) \delta 8.23(\mathrm{~d}, J=8.0 \mathrm{~Hz}, 1 \mathrm{H}), 8.00(\mathrm{~s}, 1 \mathrm{H}), 7.36(\mathrm{t}, J=7.7 \mathrm{~Hz}, 1 \mathrm{H})$, $7.17(\mathrm{~d}, J=7.1 \mathrm{~Hz}, 1 \mathrm{H}), 6.71(\mathrm{~d}, J=7.8 \mathrm{~Hz}, 1 \mathrm{H}), 6.29-6.23(\mathrm{~m}, 3 \mathrm{H}), 5.93(\mathrm{~s}, 2 \mathrm{H}), 5.66(\mathrm{~d}, J=$ $6.8 \mathrm{~Hz}, 1 \mathrm{H}), 3.76(\mathrm{t}, J=6.3 \mathrm{~Hz}, 1 \mathrm{H}), 3.34(\mathrm{~d}, J=6.3 \mathrm{~Hz}, 1 \mathrm{H}), 1.94(\mathrm{~s}, 3 \mathrm{H}) .{ }^{13} \mathbf{C}$ NMR (100 MHz, CDCl $\mathbf{l}_{3} \delta 175.35$ (q, $\left.J=35.1 \mathrm{~Hz}\right), 167.47,150.38,148.73,148.11,135.97$ (q, $\left.J=5.0 \mathrm{~Hz}\right)$, 
$133.25,132.53,124.83,124.80,121.65,121.47,121.26,118.94,116.98$ (q, $J=290.0 \mathrm{~Hz}$ ), 111.21, 108.98, 105.83, 101.93, 101.66, 56.97, 45.90, 27.21, 18.67. HRMS (ESI) calculated $[\mathrm{M}+\mathrm{H}]^{+}$for $\mathrm{C}_{24} \mathrm{H}_{17} \mathrm{O}_{5} \mathrm{~N}_{1} \mathrm{~F}_{3}$ : 456.1053, found: 456.1056. FTIR (cm $\left.{ }^{-1}\right)$ 3499, 3022, 1761, 1672, 1516, 1446, 1395, 1147, 1042, 862.

(7S,7aS,11aR)-10-Methyl-7-(naphthalen-2-yl)-4-(2,2,2-trifluoroacetyl)-7a,11a-dihydro7H,8H-pyrano[3,4-c]pyrrolo[3,2,1-ij]quinolin-8-one (3s)

Following the general procedure, treatment of $(E)$-3-(naphthalen-<smiles>CC1=C[C@H]2c3cccc4c(C(=O)C(F)(F)F)cn(c34)[C@H](c3ccc4ccccc4c3)[C@H]2C(=O)O1</smiles2-yl)acrylaldehyde $2 \mathrm{~s}(68.3 \mathrm{mg}, 0.375 \mathrm{mmol})$ and $(E)-4-(3-(2,2,2-$ trifluoroacetyl)-1H-indol-7-yl)but-3-en-2-one 1d (70.0 mg, 0.25 mmol) with triazolium salt $4(13.8 \mathrm{mg}, 0.037 \mathrm{mmol})$, oxidant 5 $(153.0 \mathrm{mg}, 0.375 \mathrm{mmol})$ and DABCO $(28.0 \mathrm{mg}, 0.25 \mathrm{mmol})$ in $\operatorname{DMSO}(2.0 \mathrm{~mL})$ and stirring the reaction mixture at $25^{\circ} \mathrm{C}$ for $12 \mathrm{~h}$ followed by flash column chromatography (Pet. ether- EtOAc:

9:1) to afford $(7 S, 7 \mathrm{a} S, 11 \mathrm{a} R)$-10-methyl-7-(naphthalen-2-yl)-4-(2,2,2-trifluoroacetyl)-7a,11adihydro-7H,8H-pyrano[3,4-c]pyrrolo[3,2,1-ij]quinolin-8-one 3s as a brown solid $(88.0 \mathrm{mg}, 77 \%$ yield).

$\boldsymbol{R}_{\boldsymbol{f}}($ Pet. ether $/ \mathrm{EtOAc}=80 / 20): 0.42$; er $=95: 5,[\alpha]_{\mathrm{D}}{ }^{25}=166.48\left(\mathrm{c} 1.0, \mathrm{CHCl}_{3}\right)$. HPLC $($ Chiralpak IA, 90:10 Hexane / IPA, $1.0 \mathrm{~mL} / \mathrm{min}$ ) Minor: $18.8 \mathrm{~min}$, Major: $20.5 \mathrm{~min}$.

${ }^{1} \mathbf{H}$ NMR (400 MHz, CDCl $) \delta 8.31(\mathrm{~d}, J=7.8 \mathrm{~Hz}, 1 \mathrm{H}), 8.08(\mathrm{~s}, 1 \mathrm{H}), 7.85(\mathrm{t}, J=7.5 \mathrm{~Hz}, 2 \mathrm{H})$, $7.65(\mathrm{~d}, J=7.8 \mathrm{~Hz}, 1 \mathrm{H}), 7.49(\mathrm{~m}, 2 \mathrm{H}), 7.41(\mathrm{t}, J=7.7 \mathrm{~Hz}, 1 \mathrm{H}), 7.20(\mathrm{~d}, J=7.2 \mathrm{~Hz}, 1 \mathrm{H}), 7.10$ (d, $J=10.1 \mathrm{~Hz}, 2 \mathrm{H}), 6.53(\mathrm{~s}, 1 \mathrm{H}), 5.64(\mathrm{~d}, J=6.9 \mathrm{~Hz}, 1 \mathrm{H}), 3.74(\mathrm{t}, J=6.4 \mathrm{~Hz}, 1 \mathrm{H}), 3.52$ (d, $J=6.3$ Hz, 1H), 1.96 (s, 3H). ${ }^{13} \mathbf{C}$ NMR (100 MHz, $\left.\mathbf{C D C l}_{3}\right) \delta 174.44$ (q, $\left.J=35.19 \mathrm{~Hz}\right), 166.51,149.45$, $135.17,132.43,132.23,132.20,128.72$, 127.06, 126.85, 126.14, 126.01, 123.90, 123.59, 121.74, 120.71, 121.72 120.56, 120.40, 120.34, 116.0 (q, $J=290.92 \mathrm{~Hz}), 110.33,100.93,56.42$, 44.77, 26.23, 17.72. HRMS (ESI) calculated $[\mathrm{M}+\mathrm{H}]^{+}$for $\mathrm{C}_{27} \mathrm{H}_{19} \mathrm{O}_{3} \mathrm{NF}_{3}: 462.1312$, found: 462.1316 . FTIR (cm-1) 3127, 3057, 2924, 1762, 1672, 1516, 1452, 1146, 1049, 906. 
(7S,7aS,11aR)-7-(Furan-2-yl)-10-methyl-4-(2,2,2-trifluoroacetyl)-7,7a-dihydropyrano[3,4c]pyrrolo[3,2,1-ij]quinolin-8(11aH)-one (3t)

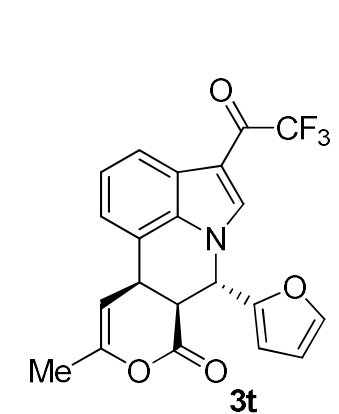

Following the general procedure, treatment of $(E)-3$-(furan-2yl)acrylaldehyde $2 \mathrm{t} \quad(46.0 \mathrm{mg}, 0.375 \mathrm{mmol})$ and $(E)-4-(3-(2,2,2-$ trifluoroacetyl)-1H-indol-7-yl)but-3-en-2-one 1 d (70.0 mg, $0.25 \mathrm{mmol})$ with triazolium salt $4(15.0 \mathrm{mg}, 0.037 \mathrm{mmol})$, oxidant $5(153.0 \mathrm{mg}$, $0.375 \mathrm{mmol})$ and DABCO $(28.0 \mathrm{mg}, 0.25 \mathrm{mmol})$ in DMSO $(2.0 \mathrm{~mL})$ and stirring the reaction mixture at $25{ }^{\circ} \mathrm{C}$ for $12 \mathrm{~h}$ followed by flash column chromatography (Pet. ether- EtOAc: 9:1) to afford (7S,7aS,11aR)-7-(furan-2-yl)-10methyl-4-(2,2,2-trifluoroacetyl)-7,7a-dihydropyrano[3,4-c]pyrrolo[3,2,1-ij]quinolin-8(11aH)-one $3 \mathrm{t}$ as a white solid $(67.0 \mathrm{mg}, 67 \%$ yield).

$\boldsymbol{R}_{\boldsymbol{f}}($ Pet. ether $/$ EtOAc $=80 / 20): 0.40 ;$ er $=96: 4,[\alpha]_{\mathrm{D}}{ }^{25}=+152.7\left(\mathrm{c} 0.1, \mathrm{CHCl}_{3}\right)$. HPLC $($ Chiralpak AD, 90:10 Hexane / IPA, $1.0 \mathrm{~mL} / \mathrm{min}$ ) Minor: $8.0 \mathrm{~min}$, Major: $9.5 \mathrm{~min}$.

${ }^{1}$ H NMR (400 MHz, CDCl $) \delta 8.19(\mathrm{~d}, J=8.0 \mathrm{~Hz}, 1 \mathrm{H}), 8.07(\mathrm{~d}, J=1.4 \mathrm{~Hz}, 1 \mathrm{H}), 7.38(\mathrm{~d}, J=$ $1.2 \mathrm{~Hz}, 2 \mathrm{H}), 7.33$ (t, $J=7.7 \mathrm{~Hz}, 1 \mathrm{H}), 7.16$ (d, $J=7.4 \mathrm{~Hz}, 1 \mathrm{H}), 6.34(\mathrm{~s}, 1 \mathrm{H}), 6.30$ (dd, $J_{l}=3.2 \mathrm{~Hz}$ $\left., J_{2}=1.9 \mathrm{~Hz}, 1 \mathrm{H}\right), 5.95(\mathrm{~d}, J=3.2 \mathrm{~Hz}, 1 \mathrm{H}), 5.72(\mathrm{~d}, J=7.0 \mathrm{~Hz}, 1 \mathrm{H}), 3.99(\mathrm{t}, J=6.7 \mathrm{~Hz}, 1 \mathrm{H})$, $3.68\left(\mathrm{dd}, J_{1}=6.6 \mathrm{~Hz}, J_{2}=2.1 \mathrm{~Hz}, 1 \mathrm{H}\right), 1.96(\mathrm{~s}, 3 \mathrm{H}) .{ }^{13} \mathbf{C} \mathbf{N M R}\left(\mathbf{1 0 0} \mathbf{M H z}, \mathbf{C D C l}_{3}\right) \delta 175.36(\mathrm{q}$, $J=35.3 \mathrm{~Hz}), 167.06,150.44,150.41,143.63,135.93$ (q, $J=5.0 \mathrm{~Hz}), 132.65,124.92,124.83$, $121.55,121.38,120.98,115.50(\mathrm{q}, J=290.0 \mathrm{~Hz}), 111.23,110.83,108.24,101.88,51.85,42.63$, 28.25, 18.75. HRMS (ESI) calculated $[\mathrm{M}+\mathrm{H}]^{+}$for $\mathrm{C}_{23} \mathrm{H}_{17} \mathrm{O}_{3} \mathrm{~N}_{1} \mathrm{~F}_{3}$ : 402.0948, found: 402.0951 . FTIR (cm $\left.{ }^{-1}\right)$ 2926, 1762, 1669, 1517, 1389, 1286, 1147, 1046, 996.

\section{(7S,7aS,11aR)-10-Methyl-7-(thiophen-2-yl)-4-(2,2,2-trifluoroacetyl)-7,7a-}

\section{dihydropyrano[3,4-c]pyrrolo[3,2,1-ij]quinolin-8(11aH)-one (3u)}<smiles>CC1=C[C@H]2c3cccc4c(C(=O)C(F)(F)F)cn(c34)[C@H](c3cccs3)[C@H]2C(=O)O1</smiles>

Following the general procedure, treatment of $(E)$-3-(thiophen-2yl)acrylaldehyde $\quad \mathbf{2 u} \quad(52.0 \quad \mathrm{mg}, \quad 0.375 \quad \mathrm{mmol})$ and $(E)-4-(3-(2,2,2-$ trifluoroacetyl)-1H-indol-7-yl)but-3-en-2-one 1 d $(70.0 \mathrm{mg}, 0.25 \mathrm{mmol})$ with triazolium salt $4(15.0 \mathrm{mg}, 0.037 \mathrm{mmol})$, oxidant $5(153.0 \mathrm{mg}, 0.375$ $\mathrm{mmol})$ and DABCO $(28.0 \mathrm{mg}, 0.25 \mathrm{mmol})$ in DMSO $(2.0 \mathrm{~mL})$ and stirring the reaction mixture at $25{ }^{\circ} \mathrm{C}$ for $12 \mathrm{~h}$ followed by flash column chromatography (Pet. ether- EtOAc: 9:1) to afford (7S,7aS,11aR)-10-methyl-7-(thiophen-2-yl)- 
4-(2,2,2-trifluoroacetyl)-7,7a-dihydropyrano[3,4-c]pyrrolo[3,2,1-ij]quinolin-8(11a $H)$-one $\mathbf{3 u}$ as a white solid (84.0 $\mathrm{mg}, 81 \%$ yield).

$\boldsymbol{R}_{\boldsymbol{f}}($ Pet. ether $/$ EtOAc $=80 / 20): 0.40$; er $=90: 10,[\alpha]_{\mathrm{D}}{ }^{25}=+150.1 \quad\left(\mathrm{c} 0.1, \mathrm{CHCl}_{3}\right)$. HPLC (Chiralpak AD, 90:10 Hexane / IPA, $1.0 \mathrm{~mL} / \mathrm{min}$ ) Minor: $9.0 \mathrm{~min}$, Major: $11.9 \mathrm{~min}$.

${ }^{1} \mathbf{H}$ NMR (400 MHz, CDCl $) \delta 8.22(\mathrm{~d}, J=7.9 \mathrm{~Hz}, 1 \mathrm{H}), 8.09(\mathrm{~s}, 1 \mathrm{H}), 7.36(\mathrm{t}, J=7.7 \mathrm{~Hz}, 1 \mathrm{H})$, $7.27(\mathrm{~d}, J=4.4 \mathrm{~Hz}, 1 \mathrm{H}), 7.17(\mathrm{~d}, J=7.2 \mathrm{~Hz}, 1 \mathrm{H}), 6.91(\mathrm{~s}, 1 \mathrm{H}), 6.56$ (s, 2H), 5.71 (d, $J=6.8 \mathrm{~Hz}$, 1H), 3.94 (t, $J=6.3 \mathrm{~Hz}, 1 \mathrm{H}), 3.51$ (d, $J=6.1 \mathrm{~Hz}, 1 \mathrm{H}), 1.95$ (s, 3H). ${ }^{13}$ C NMR (100 MHz, $\left.\mathbf{C D C l}_{3}\right) \delta 175.36(\mathrm{q}, J=35.1 \mathrm{~Hz}), 167.00,150.40,141.27,135.90,135.85,132.58,127.60$, $126.11,125.38,124.92,121.66,121.45,121.25,120.92,116.98$ (q, $J=291.0 \mathrm{~Hz}), 111.26$, 101.87, 53.76, 45.87, 27.57, 18.71, 18.68. HRMS (ESI) calculated $[\mathrm{M}+\mathrm{H}]^{+}$for $\mathrm{C}_{21} \mathrm{H}_{15} \mathrm{O}_{4} \mathrm{~N}_{1} \mathrm{~F}_{3} \mathrm{~S}$ : 418.0719, found: 418.0724. FTIR (cm $\left.\mathbf{( c m}^{-1}\right)$ 3440, 2924, 1761, 1672, 1517, 1451, 1391, 1147, 1077, $1047,1012,905$.

(7R,7aS,11a $R)$-10-Methyl-7-((E)-styryl)-4-(2,2,2-trifluoroacetyl)-7a,11a-dihydro-7H,8Hpyrano[3,4-c]pyrrolo[3,2,1-ij]quinolin-8-one (3v)<smiles>CC1=C[C@H]2c3cccc4c(C(=O)C(F)(F)F)cn(c34)[C@H](/C=C/c3ccccc3)[C@H]2C(=O)O1</smiles>

Following the general procedure, treatment of $(2 E, 4 E)-5-$ phenylpenta-2,4-dienal $2 \mathbf{v}(59.3 \mathrm{mg}, 0.375 \mathrm{mmol})$ and $(E)-4-(3-$ (2,2,2-trifluoroacetyl)-1H-indol-7-yl)but-3-en-2-one 1 d $(70.0 \mathrm{mg}$, $0.25 \mathrm{mmol})$ with triazolium salt $4(15.0 \mathrm{mg}, 0.037 \mathrm{mmol})$, oxidant $5(153.0 \mathrm{mg}, 0.375 \mathrm{mmol})$ and DABCO $(28.0 \mathrm{mg}, 0.25 \mathrm{mmol})$ in DMSO $(2.0 \mathrm{~mL})$ and stirring the reaction mixture at $25{ }^{\circ} \mathrm{C}$ for $12 \mathrm{~h}$ followed by flash column chromatography (Pet. ether- EtOAc: 9:1) to afford (7R,7aS,11aR)-10methyl-7-((E)-styryl)-4-(2,2,2-trifluoroacetyl)-7a,11a-dihydro-7H,8H-pyrano[3,4c]pyrrolo[3,2,1-ij]quinolin-8-one 3v as a white solid (101.0 $\mathrm{mg}, 93 \%$ yield). $\boldsymbol{R}_{\boldsymbol{f}}$ (Pet. ether $/$ EtOAc $\left.=80 / 20\right): 0.35$; er $=96: 4,[\alpha]_{\mathrm{D}}^{25}=155.16$ (c 1.0, $\left.\mathrm{CHCl}_{3}\right)$. HPLC (ChiralpakAD, 90:10 Hexane / IPA, $1.0 \mathrm{~mL} / \mathrm{min}$ ) Minor: $10.6 \mathrm{~min}$, Major: $13.0 \mathrm{~min}$.

${ }^{1} \mathbf{H}$ NMR (400 MHz, $\left.\mathbf{C D C l}_{3}\right) \delta 8.23(\mathrm{~d}, J=8.0 \mathrm{~Hz}, 1 \mathrm{H}), 8.06(\mathrm{~s}, 1 \mathrm{H}), 7.36(\mathrm{t}, \mathrm{J}=7.7 \mathrm{~Hz}, 1 \mathrm{H})$, $7.29(\mathrm{~s}, 5 \mathrm{H}), 6.32\left(\mathrm{dd}, J_{1}=5.8 \mathrm{~Hz}, J_{2}=15.8 \mathrm{~Hz}, 2 \mathrm{H}\right), 6.16(\mathrm{~d}, J=15.6 \mathrm{~Hz}, 1 \mathrm{H}), 5.85(\mathrm{~s}, 1 \mathrm{H}), 5.70$ $(\mathrm{d}, J=6.6 \mathrm{~Hz}, 1 \mathrm{H}), 4.03(\mathrm{t}, J=6.2 \mathrm{~Hz}, 1 \mathrm{H}), 3.37(\mathrm{~d}, \mathrm{~J}=6.3 \mathrm{~Hz}, 1 \mathrm{H}), 1.97(\mathrm{~s}, 3 \mathrm{H}) .{ }^{13} \mathbf{C} \mathbf{N M R}$ $\left(100 \mathrm{MHz}, \mathbf{C D C l}_{3}\right) \delta 174.53(\mathrm{q}, J=35.2 \mathrm{~Hz}), 166.14,149.39,134.87(\mathrm{q}, J=4.9 \mathrm{~Hz}), 133.96$, $132.88,131.66,127.89,125.88,124.16,124.14,123.85,120.53,120.50,120.18,120.41,116.08$ 
(q, $J=290.9 \mathrm{~Hz}), 110.04,101.05,54.67,42.55,27.02,17.76$. HRMS (ESI) calculated $[\mathrm{M}+\mathrm{H}]^{+}$ for $\mathrm{C}_{25} \mathrm{H}_{19} \mathrm{O}_{3} \mathrm{NF}_{3}$ : 438.1312, found: 438.1315. FTIR (cm $\left.{ }^{-1}\right)$ 3435, 3027, 2924, 1762, 1670, 1516, $1451,1392,1148,838$.

(7S,7aS,11aR)-10-Cyclopropyl-7-phenyl-4-(2,2,2-trifluoroacetyl)-7,7a-dihydropyrano[3,4c]pyrrolo[3,2,1-ij]quinolin-8(11aH)-one (3w)<smiles>O=C1OC(C2CC2)=CC2c3ccccc3[C@@H]1n1cc(C(=O)C(F)(F)F)c3cccc2c31</smiles>

Following the general procedure, treatment of cinnamaldehyde $\mathbf{2 a}(50.0$ $\mathrm{mg}, \quad 47 \mu \mathrm{L}, \quad 0.375 \quad \mathrm{mmol})$ and (E)-1-cyclopropyl-3-(3-(2,2,2trifluoroacetyl)-1H-indol-7-yl)prop-2-en-1-one 1 w (76.0 mg, $0.25 \mathrm{mmol}$ ) with triazolium salt 4 (15.0 mg, $0.037 \mathrm{mmol})$, oxidant 5 (153.0 mg, 0.375 $\mathrm{mmol})$ and DABCO $(28.0 \mathrm{mg}, 0.25 \mathrm{mmol})$ in DMSO $(2.0 \mathrm{~mL})$ and stirring the reaction mixture at $25^{\circ} \mathrm{C}$ for $12 \mathrm{~h}$ followed by flash column chromatography (Pet. ether- EtOAc: 9:1) to afford (7S,7aS,11aR)-10-cyclopropyl-7-phenyl-4(2,2,2-trifluoroacetyl)-7,7a-dihydropyrano[3,4-c]pyrrolo[3,2,1-ij]quinolin-8(11a $H)$-one $\mathbf{3 w}$ as a white solid (104.0 mg, 95\% yield).

$\boldsymbol{R}_{\boldsymbol{f}}($ Pet. ether $/$ EtOAc $=80 / 20): 0.50$; er $=99: 1,[\alpha]_{\mathrm{D}}{ }^{25}=+146.6\left(\mathrm{c} 0.1, \mathrm{CHCl}_{3}\right)$. HPLC $($ Chiralpak AD, 90:10 Hexane / IPA, 1.0 mL/min) Minor: $6.6 \mathrm{~min}$, Major: $11.3 \mathrm{~min}$.

${ }^{1}$ H NMR (400 MHz, CDCl $) \delta 8.25(\mathrm{~d}, J=7.7 \mathrm{~Hz}, 1 \mathrm{H}), 8.02(\mathrm{~s}, 1 \mathrm{H}), 7.40-7.33(\mathrm{~m}, 4 \mathrm{H}), 7.19(\mathrm{~d}$, $J=7.2 \mathrm{~Hz}, 1 \mathrm{H}), 6.84-6.82(\mathrm{~m}, 2 \mathrm{H}), 6.34(\mathrm{~s}, 1 \mathrm{H}), 5.69$ (d, $J=7.1 \mathrm{~Hz}, 1 \mathrm{H}), 3.71(\mathrm{t}, J=6.5 \mathrm{~Hz}$, 1H), 3.41-3.39 (m, 1H), 1.57-1.52 (m, 1H), 0.74-0.72 (m, 1H). ${ }^{13} \mathbf{C}$ NMR (100 MHz, CDCl $) \delta$ 175.29 (q, $J=35.3 \mathrm{~Hz}$ ), 167.41, 153.91, 138.79, 136.06, 133.36, 129.48, 128.86, 125.28, 124.86, 124.79, 121.69, 121.56, 121.35, 116.97 (q, $J=291.0 \mathrm{~Hz}$ ), 111.15, 99.55, 57.30, 46.05, 27.03, 12.67, 5.13, 4.81. HRMS (ESI) calculated $[\mathrm{M}+\mathrm{H}]^{+}$for $\mathrm{C}_{25} \mathrm{H}_{19} \mathrm{O}_{3} \mathrm{~N}_{1} \mathrm{~F}_{3}$ : 438.1312, found: 438.1318. FTIR (cm-1) 2078, 1761, 1668, 1517, 1452, 1392, 1349, 1143, 1076, 967.

\section{(7S,7aS,11aR)-10-Isobutyl-7-phenyl-4-(2,2,2-trifluoroacetyl)-7,7a-dihydropyrano[3,4-} c]pyrrolo[3,2,1-ij]quinolin-8(11aH)-one (3x)

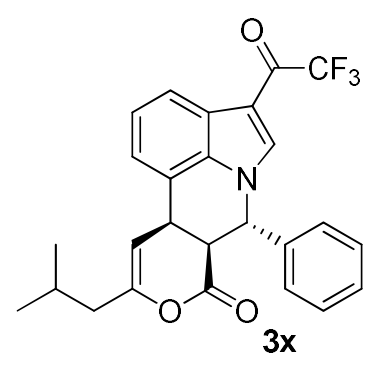

Following the general procedure, treatment of cinnamaldehyde $\mathbf{2 a}$ (40.0 $\mathrm{mg}, 38 \mu \mathrm{L}, 0.3 \mathrm{mmol})$ and (E)-5-methyl-1-(3-(2,2,2-trifluoroacetyl)-1 $H$ indol-7-yl)hex-1-en-3-one 1x (65.0 mg, $0.20 \mathrm{mmol})$ with triazolium salt 4 (11.0 mg, $0.03 \mathrm{mmol})$, oxidant 5 (123.0 $\mathrm{mg}, 0.3 \mathrm{mmol})$ and DABCO 
$(23.0 \mathrm{mg}, 0.2 \mathrm{mmol})$ in DMSO $(2.0 \mathrm{~mL})$ and stirring the reaction mixture at $25{ }^{\circ} \mathrm{C}$ for $12 \mathrm{~h}$ followed by flash column chromatography (Pet. ether- EtOAc: 9:1) to afford (7S,7aS,11aR)-10isobutyl-7-phenyl-4-(2,2,2-trifluoroacetyl)-7,7a-dihydropyrano[3,4-c]pyrrolo[3,2,1-ij]quinolin$8(11 \mathrm{a} H)$-one $\mathbf{3 x}$ as a white solid $(69.0 \mathrm{mg}, 76 \%$ yield).

$\boldsymbol{R}_{\boldsymbol{f}}($ Pet. ether $/ \mathrm{EtOAc}=80 / 20): 0.60 ;$ er $=96: 4,[\alpha]_{\mathrm{D}}{ }^{25}=+113.5\left(\mathrm{c} 0.2, \mathrm{CHCl}_{3}\right)$. HPLC $($ Chiralpak AD, 90:10 Hexane / IPA, $1.0 \mathrm{~mL} / \mathrm{min}$ ) Minor: 7.7 min, Major: $9.9 \mathrm{~min}$.

${ }^{1}$ H NMR (400 MHz, CDCl $) \delta 8.26(\mathrm{~d}, J=8.0 \mathrm{~Hz}, 1 \mathrm{H}), 8.02(\mathrm{~d}, J=1.1 \mathrm{~Hz}, 1 \mathrm{H}), 7.38(\mathrm{t}, J=7.7$ $\mathrm{Hz}, 1 \mathrm{H}), 7.35-7.33(\mathrm{~m}, 3 \mathrm{H}), 7.19(\mathrm{~d}, J=7.4 \mathrm{~Hz}, 1 \mathrm{H}), 6.84\left(\mathrm{dd}, J_{l}=6.6, J_{2}=2.7 \mathrm{~Hz}, 1 \mathrm{H}\right), 6.36(\mathrm{~s}$, $1 \mathrm{H}), 5.67(\mathrm{~d}, J=7.1 \mathrm{~Hz}, 1 \mathrm{H}), 3.73(\mathrm{t}, J=6.7 \mathrm{~Hz}, 1 \mathrm{H}), 3.38\left(\mathrm{dd}, J_{l}=6.3, J_{2}=1.8 \mathrm{~Hz}, 1 \mathrm{H}\right), 2.13-$ $2.02(\mathrm{~m}, 2 \mathrm{H}), 1.95-1.85(\mathrm{~m}, 1 \mathrm{H}), 0.91\left(\mathrm{dd}, J_{l}=13.4, J_{2}=6.6 \mathrm{~Hz}, 6 \mathrm{H}\right) .{ }^{13} \mathbf{C}$ NMR (100 MHz, $\left.\mathbf{C D C l}_{3}\right) \delta 175.37(\mathrm{q}, J=35.4 \mathrm{~Hz}), 167.64,153.08,138.93,136.06(\mathrm{q}, J=5.0 \mathrm{~Hz}), 133.42,129.57$, 128.93, 125.39, 124.89, 121.61, 121.54, 121.45, 117.0 (q, $J=291.0 \mathrm{~Hz}), 111.26,102.70,57.31$, 45.99, 41.83, 27.08, 25.68, 22.41, 22.02. HRMS (ESI) calculated $[\mathrm{M}+\mathrm{H}]^{+}$for $\mathrm{C}_{26} \mathrm{H}_{23} \mathrm{O}_{3} \mathrm{~F}_{3} \mathrm{~N}_{1}$ : 454.1625, found: 454.1630. FTIR (cm-1) 3439, 2960, 1761, 1671, 1518, 1454, 1394, 1145, 1041, 907.

\section{(7S,7aS,11aR)-2-Bromo-10-methyl-7-phenyl-4-(2,2,2-trifluoroacetyl)-7,7a-}

\section{dihydropyrano[3,4-c]pyrrolo[3,2,1-ij]quinolin-8(11aH)-one (3y)}<smiles>CC1=CC2c3cc(Br)cc4c(C(=O)C(F)(F)F)cn(c34)[C@H](c3ccccc3)[C@H]2C(=O)O1</smiles>

Following the general procedure, treatment of cinnamaldehyde $2 \mathbf{a}(50.0 \mathrm{mg}$, $47 \mu \mathrm{L}, 0.375 \mathrm{mmol}$ ) and (E)-4-(5-bromo-3-(2,2,2-trifluoroacetyl)-1H-indol-7yl)but-3-en-2-one $\mathbf{1 y}(90.0 \mathrm{mg}, 0.25 \mathrm{mmol})$ with triazolium salt $4(15.0 \mathrm{mg}$, $0.037 \mathrm{mmol}$ ), oxidant 5 (153.0 $\mathrm{mg}, 0.375 \mathrm{mmol})$ and DABCO (28.0 mg, 0.25 $\mathrm{mmol})$ in DMSO $(2.0 \mathrm{~mL})$ and stirring the reaction mixture at $25{ }^{\circ} \mathrm{C}$ for $12 \mathrm{~h}$ followed by flash column chromatography (Pet. ether- EtOAc: 9:1) to afford (7S,7aS,11aR)-2-bromo-10-methyl-7-phenyl-4-(2,2,2-trifluoroacetyl)-7,7a-dihydropyrano[3,4c]pyrrolo[3,2,1-ij]quinolin-8(11a $H$ )-one $\mathbf{3 y}$ as a white solid (93.0 $\mathrm{mg}, 76 \%$ yield).

$\boldsymbol{R}_{\boldsymbol{f}}($ Pet. ether $/$ EtOAc $=80 / 20): 0.45$; er $=99: 1,[\alpha]_{\mathrm{D}}^{25}=+82 . .8\left(\mathrm{c} 0.1, \mathrm{CHCl}_{3}\right)$. HPLC (Chiralpak IA, 90:10 Hexane / IPA, $1.0 \mathrm{~mL} / \mathrm{min}$ ) Minor: $10.4 \mathrm{~min}$, Major: $8.5 \mathrm{~min}$.

${ }^{1}$ H NMR (400 MHz, CDCl 3$) \delta 8.50(\mathrm{~s}, 1 \mathrm{H}), 8.09(\mathrm{~s}, 1 \mathrm{H}), 7.42-7.34(\mathrm{~m}, 5 \mathrm{H}), 6.87-6.85(\mathrm{~m}, 2 \mathrm{H})$, $6.43(\mathrm{~s}, 1 \mathrm{H}), 5.71(\mathrm{~d}, J=7.1 \mathrm{~Hz}, 1 \mathrm{H}), 3.77(\mathrm{t}, J=6.6 \mathrm{~Hz}, 1 \mathrm{H}), 3.49\left(\mathrm{dd}, J_{l}=6.4, J_{2}=1.8 \mathrm{~Hz}\right.$, 1H), 2.04 (s, 3H). ${ }^{13} \mathbf{C}$ NMR (100 MHz, $\left.\mathbf{C D C l}_{3}\right) \delta 175.21$ (q, $\left.J=35.4 \mathrm{~Hz}\right), 167.13,138.41$, 
136.48 (q, $J=4.8 \mathrm{~Hz}), 132.05,129.63,129.08,126.16,125.19,124.92,124.20,123.09,118.47$, 116.82 (q, $J=291.0 \mathrm{~Hz}$ ), 110.70, 101.17, 57.28, 45.70, 26.99, 18.78. HRMS (ESI) calculated $[\mathrm{M}+\mathrm{H}]^{+}$for $\mathrm{C}_{23} \mathrm{H}_{16} \mathrm{O}_{3} \mathrm{~N}_{1} \mathrm{~F}_{3} \mathrm{Br}$ : 490.0260, found: 490.0266. FTIR (cm $\left.{ }^{-1}\right)$ 3436, 2924, 1764, 1674, 1516, 1451, 1394, 1306, 1147, 1045, 912.

\section{Functionalization of Pyrroloquinolines}

(4S,5S,6R)-Methyl 6-(2-oxopropyl)-4-phenyl-1-(2,2,2-trifluoroacetyl)-5,6-dihydro-4Hpyrrolo[3,2,1-ij]quinoline-5-carboxylate (6a)

MeOH $(2.0 \mathrm{~mL})$ was added. The resulting mixture was allowed to stir
overnight at $60{ }^{\circ} \mathrm{C}$. Then the solution was concentrated under reduced methyl 6-(2-oxopropyl)-4-phenyl-1-(2,2,2-trifluoroacetyl)-5,6-dihydro-4H-pyrrolo[3,2,1-ij] quinoline-5-carboxylate 6a (42.0 $\mathrm{mg}, 76 \%$ yield).

$\boldsymbol{R}_{\boldsymbol{f}}($ Pet. ether $/$ EtOAc $=80 / 20): 0.40 ;$ er $=95: 5,[\alpha]_{\mathrm{D}}^{25}=+20.8\left(\right.$ c $\left.0.1, \mathrm{CHCl}_{3}\right)$. HPLC (Chiralpak AD, 90:10 Hexane / IPA, $1.0 \mathrm{~mL} / \mathrm{min}$ ) Minor: $6.4 \mathrm{~min}$, Major: $8.0 \mathrm{~min}$.

${ }^{1}$ H NMR (400 MHz, CDCl$) \delta 8.19(\mathrm{~d}, J=7.9 \mathrm{~Hz}, 1 \mathrm{H}), 7.56(\mathrm{~s}, 1 \mathrm{H}), 7.42-7.41(\mathrm{~m}, 3 \mathrm{H}), 7.34$ (t, $J=7.7 \mathrm{~Hz}, 1 \mathrm{H}), 7.18-7.16(\mathrm{~m}, 3 \mathrm{H}), 5.64(\mathrm{~d}, J=8.1 \mathrm{~Hz}, 1 \mathrm{H}), 4.11(\mathrm{~s}, 1 \mathrm{H}), 3.59\left(\mathrm{dd}, J_{1}=8.6, J_{2}=\right.$ $4.3 \mathrm{~Hz}, 1 \mathrm{H}), 3.52(\mathrm{~s}, 3 \mathrm{H}), 3.16\left(\mathrm{dd}, J_{l}=18.2, J_{2}=7.4 \mathrm{~Hz}, 1 \mathrm{H}\right), 2.85(\mathrm{~d}, J=15.0 \mathrm{~Hz}, 1 \mathrm{H}), 2.15$ (s, 3H). ${ }^{13} \mathbf{C}$ NMR (100 MHz, CDCl $) \delta 205.82,175.18$ (q, $\left.J=35.3 \mathrm{~Hz}\right), 171.36,135.15$ (q, $J$ $=5.0 \mathrm{~Hz}$ ), 133.78, 129.57, 129.46, 125.09, 124.72, 124.29, 121.38, 121.30, 121.01, 116.96 (q, $J$ $=291.0 \mathrm{~Hz}$ ), 110.98, 58.90, 58.87, 52.39, 49.84, 49.79, 30.51, 29.87, 29.80. HRMS (ESI) calculated $[\mathrm{M}+\mathrm{H}]^{+}$for $\mathrm{C}_{24} \mathrm{H}_{21} \mathrm{O}_{4} \mathrm{~N}_{1} \mathrm{~F}_{3}:$ 444.1417, found: 444.1427. FTIR (cm $\left.{ }^{-1}\right)$ 3435, 2921, $1730,1669,1517,1453,1170,997,963$.

(4S,5S,6R)-N-Benzyl-6-(2-oxopropyl)-4-phenyl-1-(2,2,2-trifluoroacetyl)-5,6-dihydro-4Hpyrrolo[3,2,1-ij]quinoline-5-carboxamide (7d)

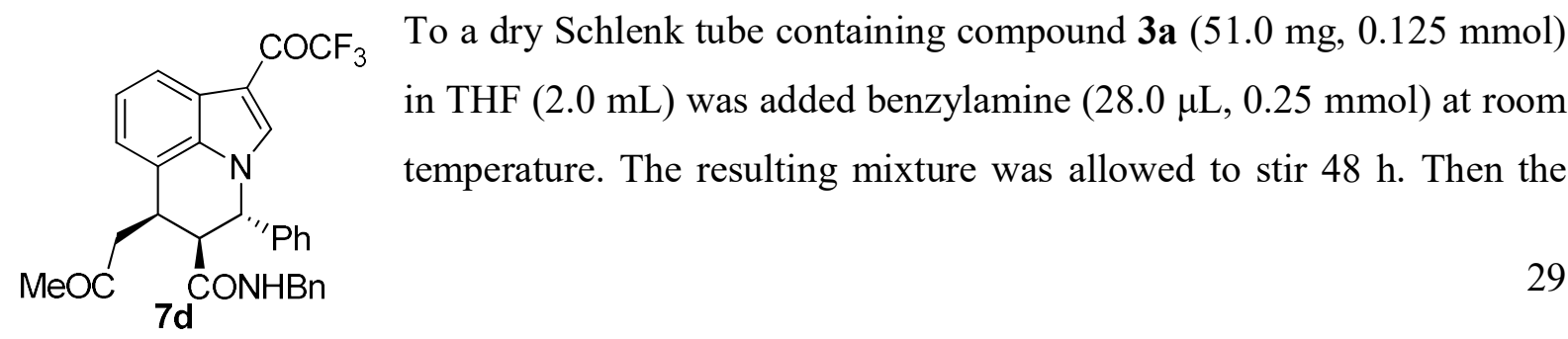


solution was concentrated under reduced pressure and the residue was purified by flash column chromatography on silica gel using petroleum ether/EtOAc $(4: 1)$ to afford the $(4 S, 5 S, 6 R)-\mathrm{N}$ benzyl-6-(2-oxopropyl)-4-phenyl-1-(2,2,2-trifluoroacetyl)-5,6-dihydro-4H-pyrrolo[3,2,1ij]quinoline-5-carboxamide $7 \mathbf{d}(52.0 \mathrm{mg}, 81 \%$ yield).

$\boldsymbol{R}_{\boldsymbol{f}}($ Pet. ether $/$ EtOAc $=80 / 20): 0.40 ;$ er $=97: 3,[\alpha]_{\mathrm{D}}^{25}=+28.7\left(\mathrm{c} 0.1, \mathrm{CHCl}_{3}\right)$. HPLC (Chiralpak AD, 90:10 Hexane / IPA, $1.0 \mathrm{~mL} / \mathrm{min}$ ) Minor: $24.3 \mathrm{~min}$, Major: $32.3 \mathrm{~min}$.

${ }^{1}$ H NMR (400 MHz, CDCl 3$) \delta 8.15(\mathrm{~d}, J=7.8 \mathrm{~Hz}, 1 \mathrm{H}), 7.48-7.13(\mathrm{~m}, 10 \mathrm{H}), 6.67(\mathrm{~d}, J=5.6 \mathrm{~Hz}$, 2H), $6.34(\mathrm{~s}, 1 \mathrm{H}), 5.54(\mathrm{t}, J=10.2 \mathrm{~Hz}, 1 \mathrm{H}), 4.37-4.23(\mathrm{~m}, 2 \mathrm{H}), 3.94(\mathrm{~d}, J=12.8 \mathrm{~Hz}, 1 \mathrm{H}), 3.44$ $\left(\mathrm{dd}, J_{1}=17.6, J_{2}=5.7 \mathrm{~Hz}, 1 \mathrm{H}\right), 3.26(\mathrm{~d}, J=6.7 \mathrm{~Hz}, 1 \mathrm{H}), 2.69-2.64(\mathrm{~m}, 1 \mathrm{H}), 2.11(\mathrm{~s}, 3 \mathrm{H}) .{ }^{13} \mathrm{C}$ NMR (100 MHz, $\left.\mathbf{C D C l}_{3}\right) \delta$ 206.18, 169.97, 137.45, 136.47, 135.34, 135.30, 133.74, 129.62, $129.44,128.60,127.34,127.07,125.21,124.72,121.96,120.86,118.37,115.48,58.69,51.90$, 46.93, 43.20, 33.05, 30.57. HRMS (ESI) calculated $[\mathrm{M}+\mathrm{H}]^{+}$for $\mathrm{C}_{30} \mathrm{H}_{26} \mathrm{O}_{3} \mathrm{~N}_{2} \mathrm{~F}_{3}:$ 519.1890,

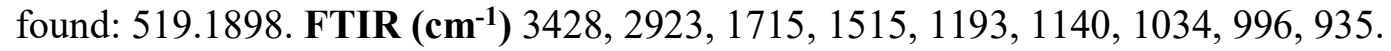

(E)-Methyl 3-(7S,7aS,11aR)-10-methyl-8-oxo-7-phenyl-4-(2,2,2-trifluoroacetyl)-7,7a,8,11atetrahydropyrano[3,4-c]pyrrolo[3,2,1-ij]quinolin-3-yl)acrylate (8d) ${ }^{6}$<smiles></smiles>

To a dry Schlenk tube containing compound 3a (51.0 mg, $0.125 \mathrm{mmol})$, $\left[\mathrm{RhCp}^{*} \mathrm{Cl}_{2}\right]_{2}$ (2.5 mol\%), AgSbF6 (10 mol\%), acrylate derivative $(0.5$ mmol, 4 equiv) and $\mathrm{Cu}(\mathrm{OAc}) 2 \cdot \mathrm{H}_{2} \mathrm{O} \quad(0.125 \mathrm{mmol}$, 1equiv $)$ and dichloroethane $(1 \mathrm{~mL})$. The Schlenk tube was placed in a pre-heated $(100$ $\left.{ }^{\circ} \mathrm{C}\right)$ oil bath. After $6 \mathrm{~h}$, the reaction mixture was cooled to room temperature, and diluted with diethyl ether and passed through a short silica gel (230-400 mesh size) bed, and repeatedly washed with diethyl ether ( $5 \mathrm{~mL} \times 3$ times). Then the solution was concentrated under reduced pressure and the residue was purified by flash column chromatography on silica gel using petroleum ether/EtOAc (5:1) to afford the $(E)$-methyl 3-(7S,7aS,11aR)-10-methyl-8-oxo-7phenyl-4-(2,2,2-trifluoroacetyl)-7,7a,8,11a-tetrahydropyrano[3,4-c]pyrrolo[3,2,1-ij]quinolin-3yl)acrylate $8 d$ ( $49.0 \mathrm{mg}, 79 \%$ yield).

$\boldsymbol{R}_{\boldsymbol{f}}($ Pet. ether $/$ EtOAc $=80 / 20): 0.40$; er $=97: 3,[\alpha]_{\mathrm{D}}{ }^{25}=-41.0\left(\mathrm{c} 0.1, \mathrm{CHCl}_{3}\right)$. HPLC $($ Chiralcel OD, 80:20 Hexane / IPA, $1.0 \mathrm{~mL} / \mathrm{min}$ ) Minor: $34.3 \mathrm{~min}$, Major: $38.3 \mathrm{~min}$.

\footnotetext{
${ }^{6}$ Lanke, V.; Bettadapur, K. R.; Prabhu, K. R. Org. Lett. 2016, 18, 5496.
} 
${ }^{1}$ H NMR (400 MHz, CDCl$) \delta 9.21(\mathrm{~d}, J=15.9 \mathrm{~Hz}, 1 \mathrm{H}), 8.12(\mathrm{~s}, 1 \mathrm{H}), 7.60(\mathrm{~d}, J=7.9 \mathrm{~Hz}, 1 \mathrm{H})$, $7.33(\mathrm{~s}, 3 \mathrm{H}), 7.20$ (d, $J=7.6 \mathrm{~Hz}, 1 \mathrm{H}), 6.80$ (d, $J=2.0 \mathrm{~Hz}, 2 \mathrm{H}), 6.35$ (d, $J=14.2 \mathrm{~Hz}, 2 \mathrm{H}), 5.66$ (d, $J=6.9 \mathrm{~Hz}, 1 \mathrm{H}), 3.85(\mathrm{~s}, 3 \mathrm{H}), 3.68$ (t, $J=6.3 \mathrm{~Hz}, 1 \mathrm{H}), 3.41$ (d, $J=6.3 \mathrm{~Hz}, 1 \mathrm{H}), 1.95$ (s, 3H). ${ }^{13}$ C NMR (100 MHz, CDCl $) \delta 174.46$ (q, $\left.J=34.4 \mathrm{~Hz}\right), 167.54,167.25,150.79,145.85$, 138.75 (q, $J=5.4 \mathrm{~Hz}$ ), 134.28, 129.63, 129.59, 125.22, 123.47, 123.30, 123.09, 122.32, 119.14, $117.48(\mathrm{q}, J=291.0 \mathrm{~Hz}), 112.04,101.57,57.37,51.85,45.35,27.09,18.74$. HRMS (ESI) calculated $[\mathrm{M}+\mathrm{Na}]^{+}$for $\mathrm{C}_{27} \mathrm{H}_{20} \mathrm{O}_{5} \mathrm{~N}_{1} \mathrm{~F}_{3} \mathrm{Na}$ : 518.1186, found: 518.1194. FTIR (cm $\left.{ }^{-1}\right)$ 3442, 3140, 2924, 1765, 1633, 1404, 1349, 1286, 1187, 1147, 981, 955.

\section{1-Benzyl-3-((7S,7aS,11aR)-10-methyl-8-oxo-7-phenyl-4-(2,2,2-trifluoroacetyl)7,7a,8,11a-}

\section{tetrahydropyrano[3,4-c]pyrrolo[3,2,1-ij]quinolin-3-yl)pyrrolidine-2,5-dione (9d) ${ }^{7}$}

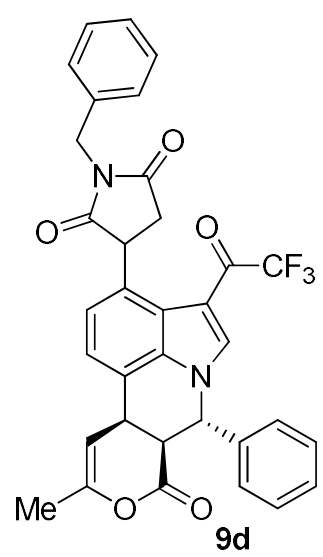

To a dry Schlenk tube containing compound 3d (51.0 mg, $0.125 \mathrm{mmol})$, $\left[\mathrm{RhCp}^{*} \mathrm{Cl}_{2}\right]_{2}(5.0 \mathrm{~mol} \%), \mathrm{AgSbF}_{6}(20 \mathrm{~mol} \%), N$-benzyl maleimide $(0.25$ mmol, 2 equiv), $\mathrm{AcOH}$ (23 mg, 3 equiv) and $\mathrm{AgOAc}(63 \mathrm{mg}, 3$ equiv) and dichloroethane $(1 \mathrm{~mL})$. The Schlenk tube was placed in a pre-heated (120 $\left.{ }^{\circ} \mathrm{C}\right)$ oil bath. After $12 \mathrm{~h}$, the reaction mixture was cooled to room temperature, and diluted with diethyl ether and passed through a short silica gel (230-400 mesh size) bed, and repeatedly washed with diethyl ether ( $5 \mathrm{~mL}$ x 3 times). Then the solution was concentrated under reduced pressure and the residue was purified by flash column chromatography on silica gel using petroleum ether/EtOAc (4:1) to afford the 1-benzyl-3-((7S,7aS,11aR)-10-methyl-8-oxo-7phenyl-4-(2,2,2-trifluoroacetyl)7,7a,8,11a tetrahydropyrano[3,4-c]pyrrolo[3,2,1-ij]quinolin-3yl)pyrrolidine-2,5-dione 9d (61.0 mg, 81\% yield) in 2:1 diastereomeric ratio (dr was determined by HPLC analysis).

$\boldsymbol{R}_{\boldsymbol{f}}($ Pet. ether $/ \mathrm{EtOAc}=70 / 30): 0.20 ;$ er $=95: 5,[\alpha]_{\mathrm{D}}{ }^{25}=+116.4\left(\mathrm{c} 0.1, \mathrm{CHCl}_{3}\right)$. HPLC $($ Chiralpak AD, 80:20 Hexane / IPA, $1.0 \mathrm{~mL} / \mathrm{min}$ ) Minor: $12.2 \mathrm{~min}$, Major: $31.7 \mathrm{~min}$, for another dr Minor: $21.4 \mathrm{~min}$, Major: $25.2 \mathrm{~min}$

${ }^{1}$ H NMR (400 MHz, CDCl $) \delta 8.12(\mathrm{~s}, 1 \mathrm{H}), 7.46(\mathrm{~d}, J=7.1 \mathrm{~Hz}, 2 \mathrm{H}), 7.37-7.29(\mathrm{~m}, 6 \mathrm{H}), 7.11$ $(\mathrm{d}, J=7.7 \mathrm{~Hz}, 1 \mathrm{H}), 6.91-6.89(\mathrm{~m}, 1 \mathrm{H}), 6.81-6.80(\mathrm{~m}, 2 \mathrm{H}), 6.35(\mathrm{~s}, 1 \mathrm{H}), 5.84(\mathrm{bs}, 1 \mathrm{H}), 5.63-5.59$

\footnotetext{
${ }^{7}$ Sherikar, M.S.; Kapanaiah, R.; Lanke, V.; Prabhu, K. R. Chem. Commun. 2018, 54, 11200.
} 
$(\mathrm{m}, 1 \mathrm{H}), 4.79(\mathrm{~s}, 1 \mathrm{H}), 3.66-3.61(\mathrm{~m}, 1 \mathrm{H}), 3.48-3.36(\mathrm{~m}, 2 \mathrm{H}), 2.70\left(\mathrm{dd}, J_{1}=30.6 \mathrm{~Hz}, J_{2}=11.8 \mathrm{~Hz}\right.$, 1H), 1.93 (s, 3H). ${ }^{13} \mathbf{C}$ NMR (100 MHz, $\left.\mathbf{C D C l}_{3}\right) \delta 178.72,176.26,175.16(\mathrm{q}, J=34.4 \mathrm{~Hz})$, $167.04,150.70,138.99,138.52,136.18,134.41,132.74,129.64,129.07,128.92,128.86,128.82$, 128.81, 128.03, 125.27, 122.92, 121.12, 117.43 (q, $J=291.0 \mathrm{~Hz}), 111.65,111.68,101.53,57.44$, 45.32, 42.80, 39.69, 26.91, 18.72. Representative Peaks of Minor Isomer: ${ }^{13}$ C NMR $\delta 178.98$, 176.20, 175.09 (q, $J=34.3 \mathrm{~Hz}$ ), 167.36, 138.42, 136.17, 134.35, 101.73, 57.47, 44.95, 30.80, 26.83. HRMS (ESI) calculated $[\mathrm{M}+\mathrm{H}]^{+}$for $\mathrm{C}_{34} \mathrm{H}_{26} \mathrm{O}_{5} \mathrm{~N}_{2} \mathrm{~F}_{3}$ : 599.1788, found: 599.1767. FTIR $\left(\mathbf{c m}^{-1}\right)$ 1957, 1766, 1703, 1588, 1513, 1401, 1340, 1309, 1149, 1077, 970, 935, 755, 701, 630. 


\section{8. ${ }^{1} \mathrm{H}$ and ${ }^{13} \mathrm{C}$ NMR Spectra of Indole Derivatives}

(E)-7-(3-Oxobut-1-en-1-yl)-1 H-indole-3-carbaldehyde (1c)
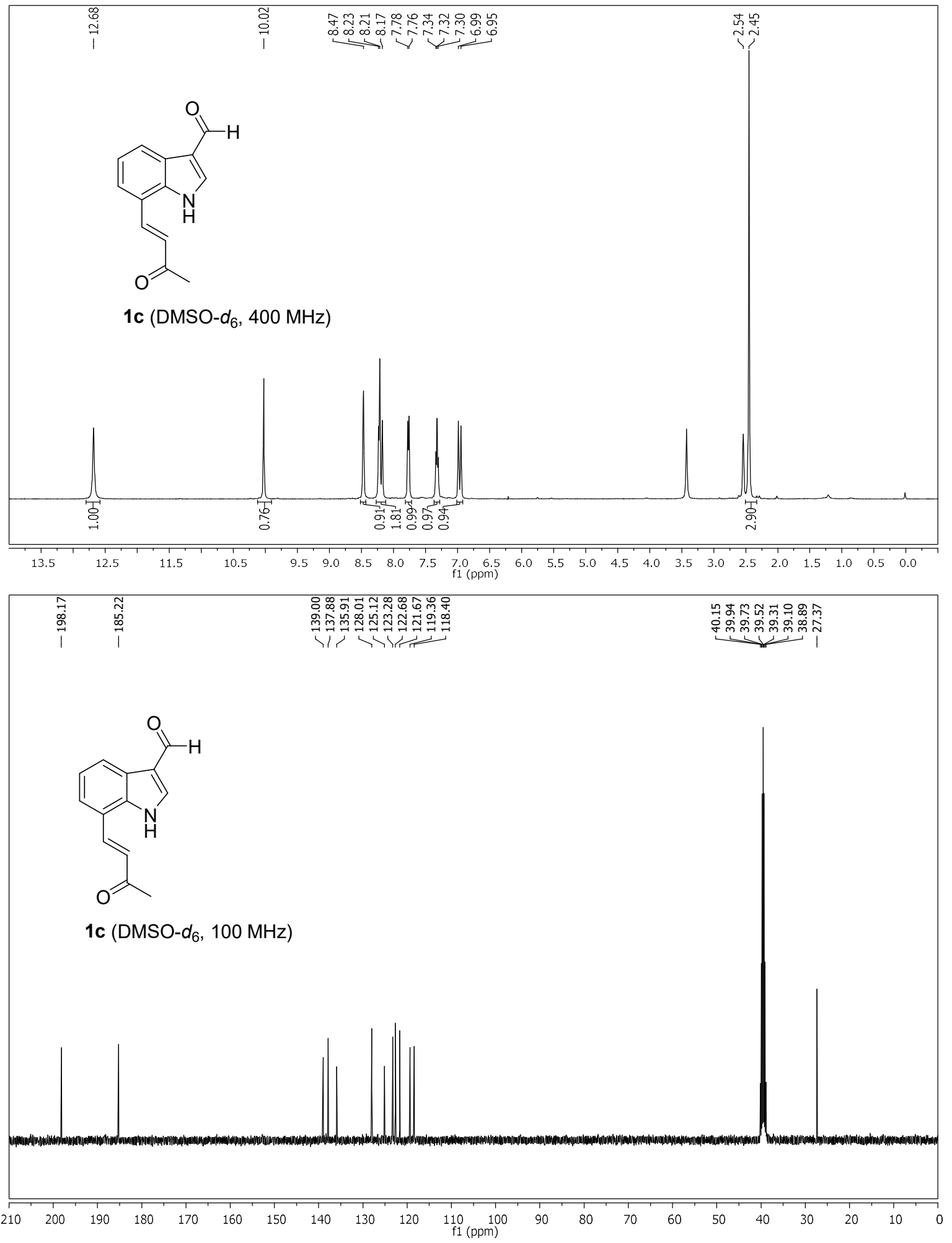
(E)-4-(3-(2,2,2-Trifluoroacetyl)-1H-indol-7-yl)but-3-en-2-one (1d)

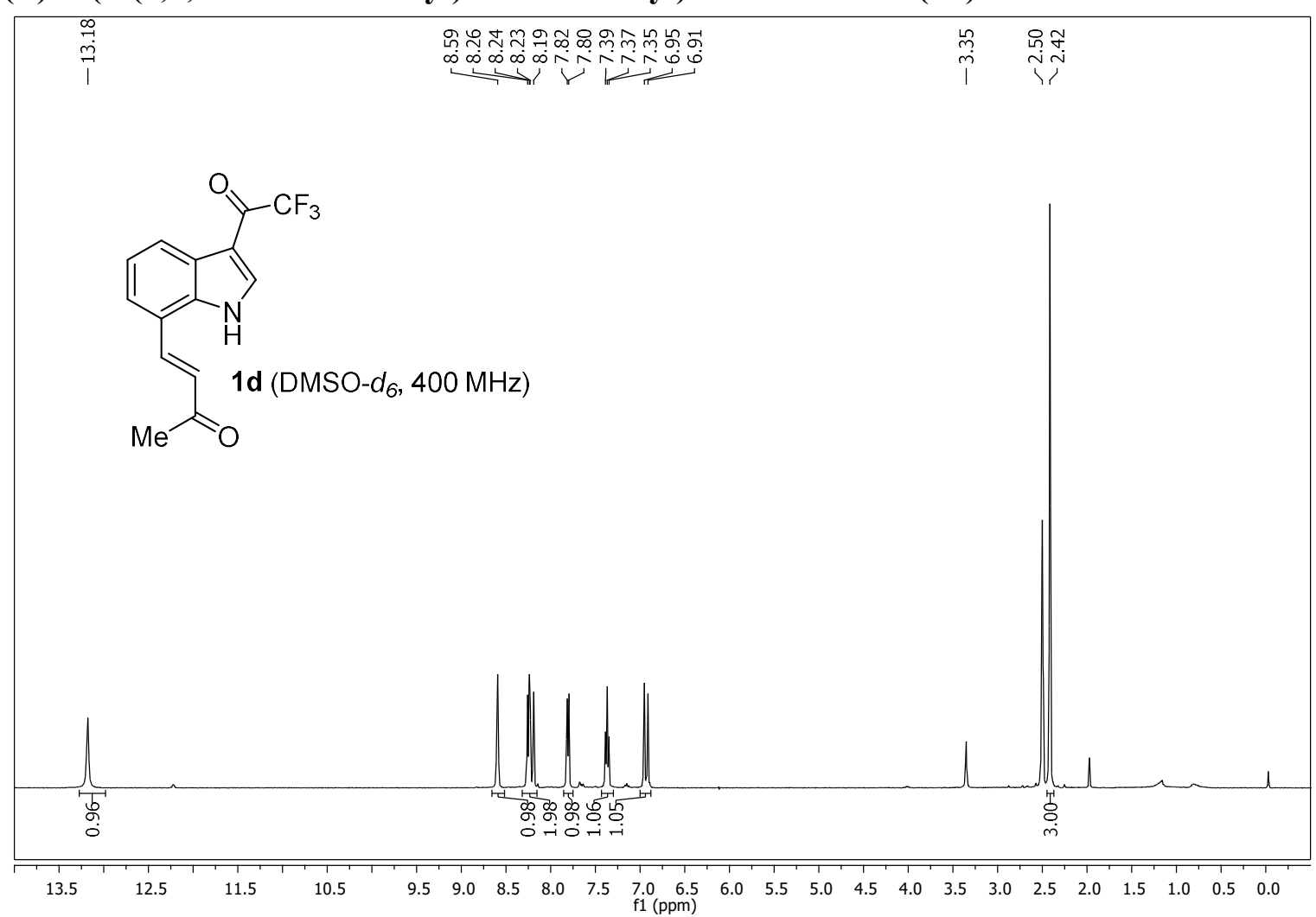

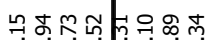 \\ ०ें कं}

and<smiles>CC(=O)/C=C/c1cccc2c(C(=O)C(F)(F)F)c[nH]c12</smiles>
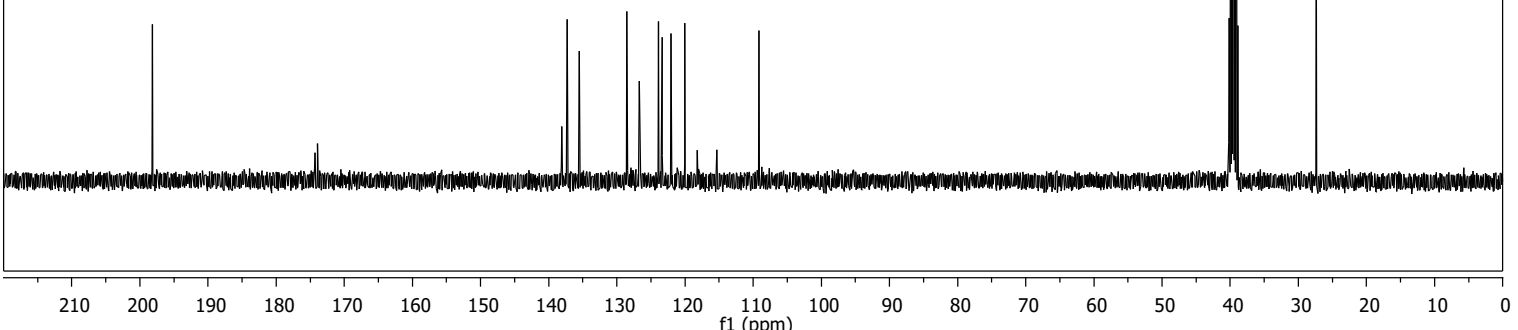
(E)-1-Cyclopropyl-3-(3-(2,2,2-trifluoroacetyl)-1H-indol-7-yl)prop-2-en-1-one (1w)
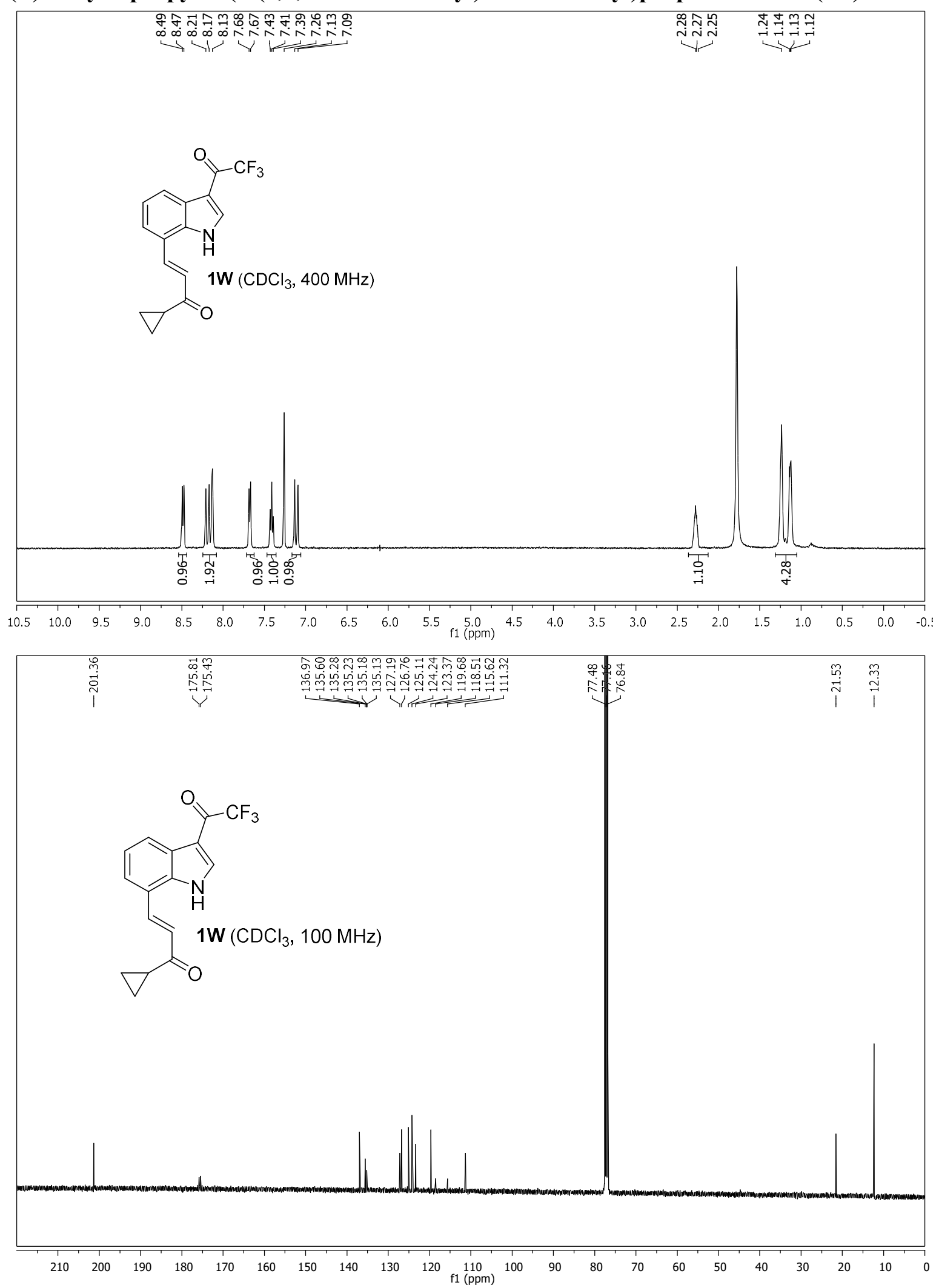
(E)-5-Methyl-1-(3-(2,2,2-trifluoroacetyl)-1H-indol-7-yl)hex-1-en-3-one (1x)
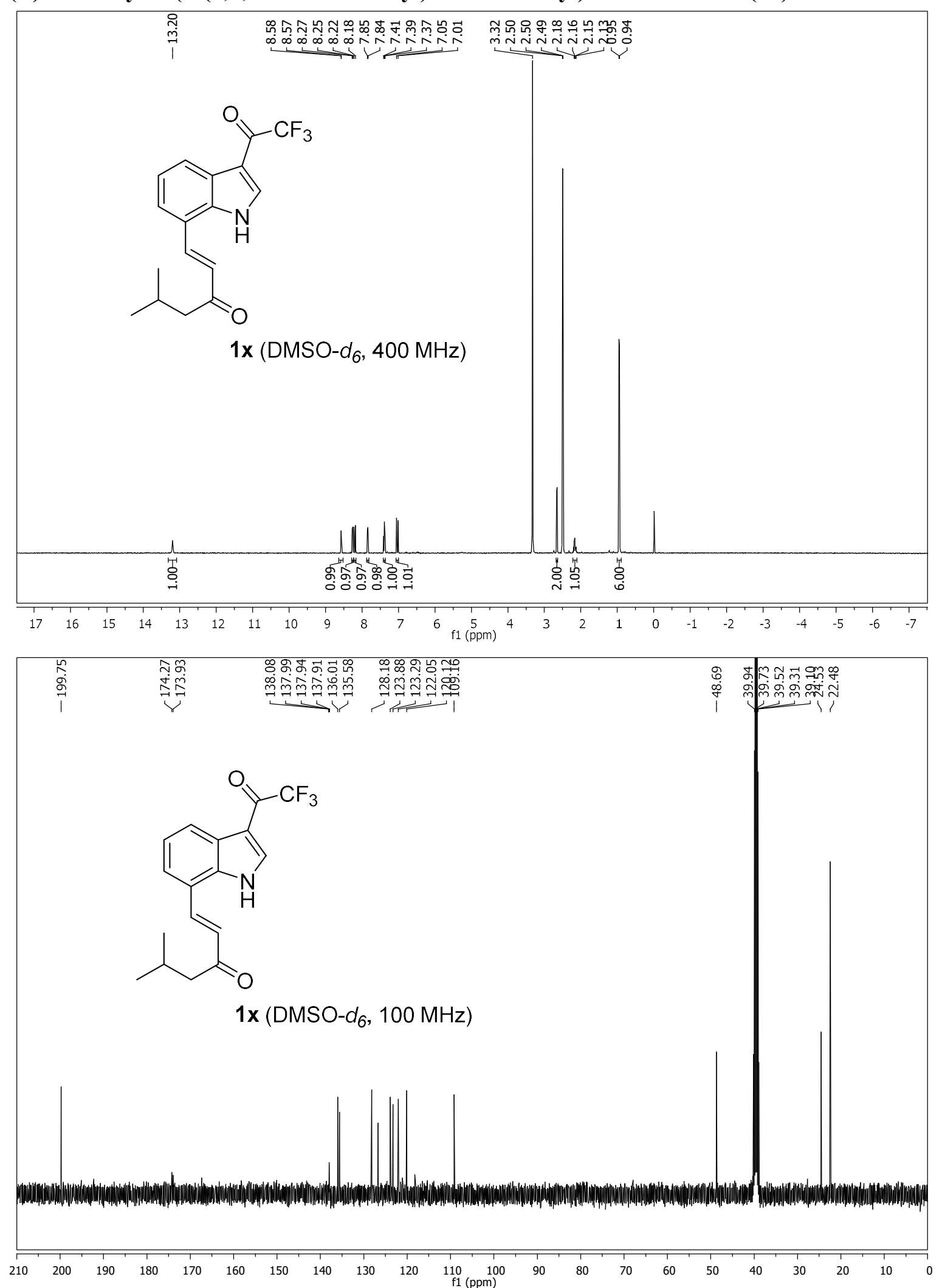
(E)-4-(5-Bromo-3-(2,2,2-trifluoroacetyl)-1 H-indol-7-yl)but-3-en-2-one (1y)
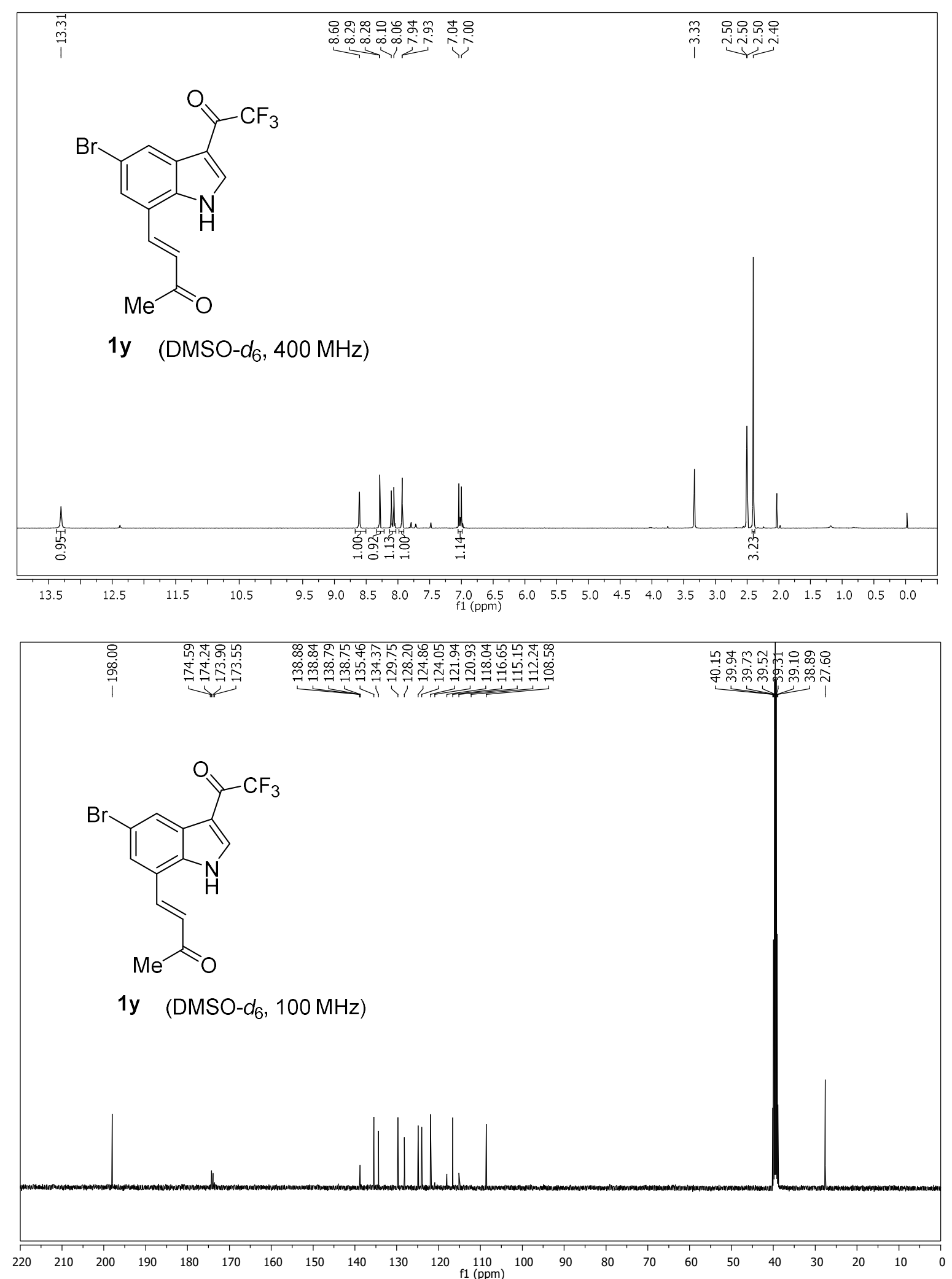


\section{9. ${ }^{1} \mathrm{H}$ and ${ }^{13} \mathrm{C}$ NMR Spectra of Pyrroloquinoline Derivatives}

(7S,7aS,11a R)-10-Methyl-8-oxo-7-phenyl-7a,11a-dihydro-7H,8H-pyrano[3,4c]pyrrolo[3,2,1-ij]quinoline-4-carbaldehyde (3c)
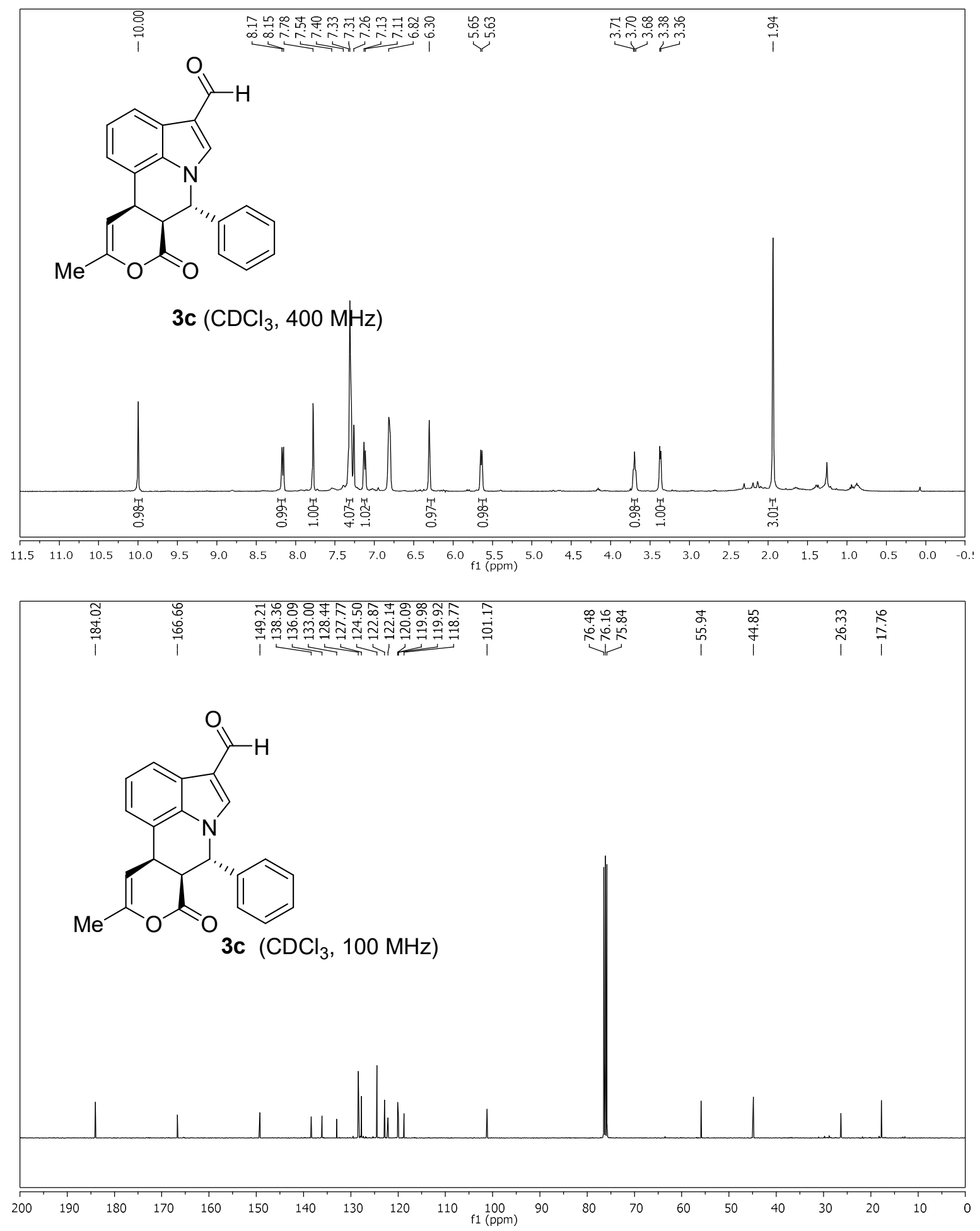
(7S,7aS,11aR)-10-Methyl-7-phenyl-4-(2,2,2-trifluoroacetyl)-7,7a-dihydropyrano[3,4c]pyrrolo[3,2,1-ij]quinolin-8(11aH)-one (3d)
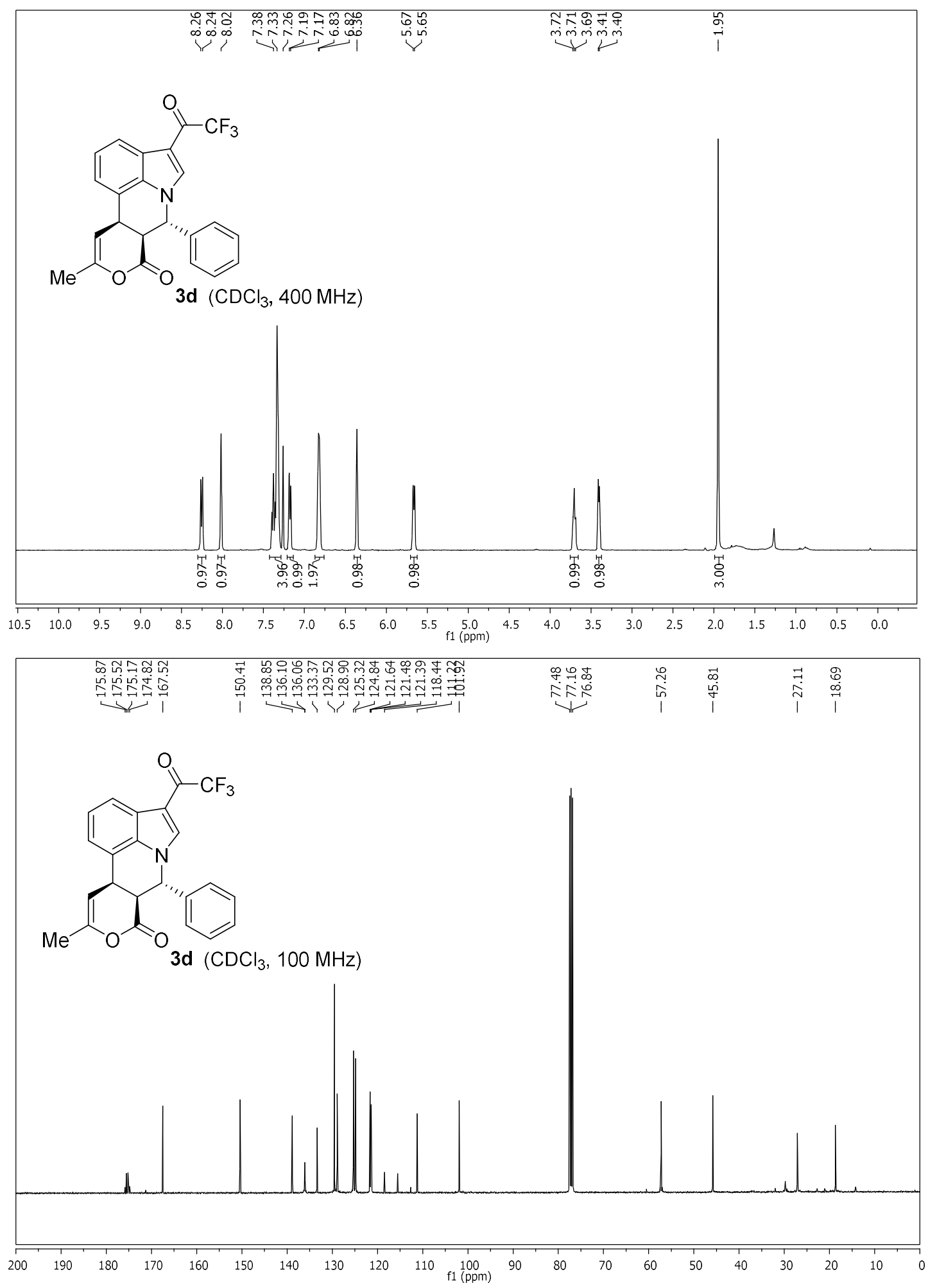

39 
(7S,7aS,11a R)-7-(4-(Dimethylamino)phenyl)-10-methyl-4-(2,2,2-trifluoroacetyl)-7,7adihydropyrano[3,4-c]pyrrolo[3,2,1-ij]quinolin-8(11aH)-one (3e)
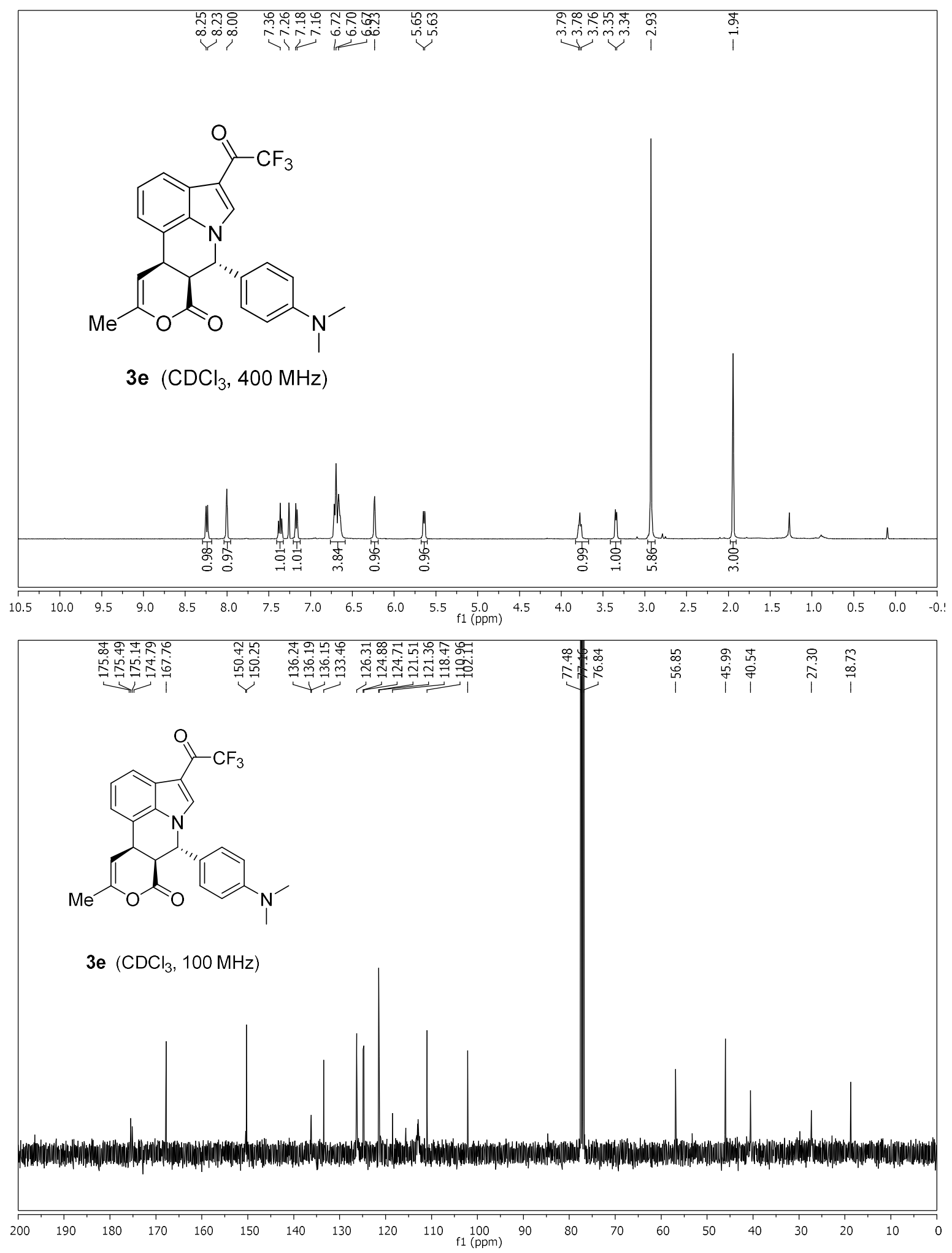
(7S,7aS,11aR)-7-(4-Methoxyphenyl)-10-methyl-4-(2,2,2-trifluoroacetyl)-7,7adihydropyrano[3,4-c]pyrrolo[3,2,1-ij]quinolin-8(11aH)-one(3f)
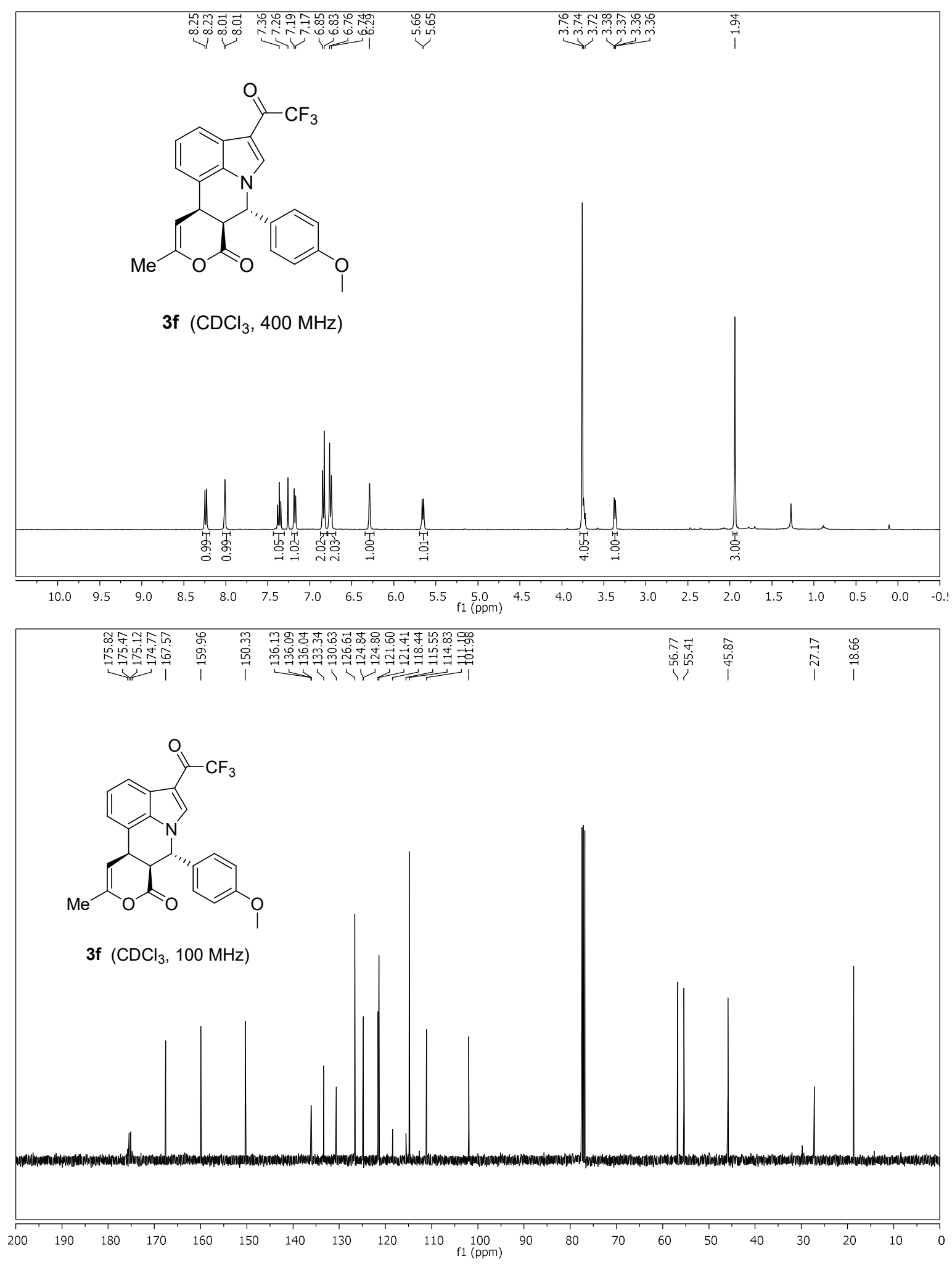

41 
7S,7aS,11a R)-10-methyl-7-(p-tolyl)-4-(2,2,2-trifluoroacetyl)-7a,11a-dihydro-7 H,8Hpyrano[3,4-c]pyrrolo[3,2,1-ij]quinolin-8-one (3g)
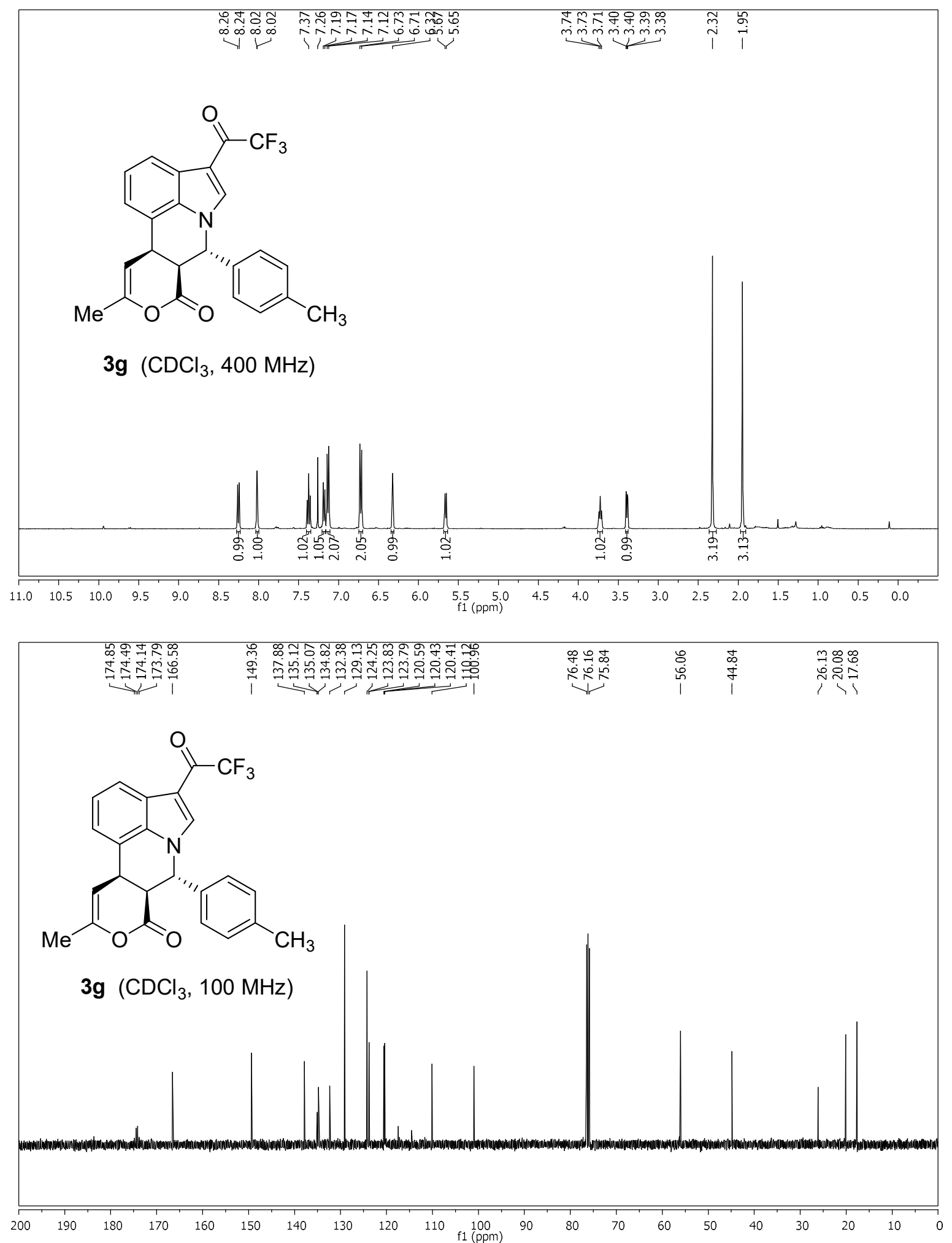
(7S,7aS,11a $R$ )-7-(4-Chlorophenyl)-10-methyl-4-(2,2,2-trifluoroacetyl)-7,7adihydropyrano[3,4-c]pyrrolo[3,2,1-ij]quinolin-8(11aH)-one (3h)
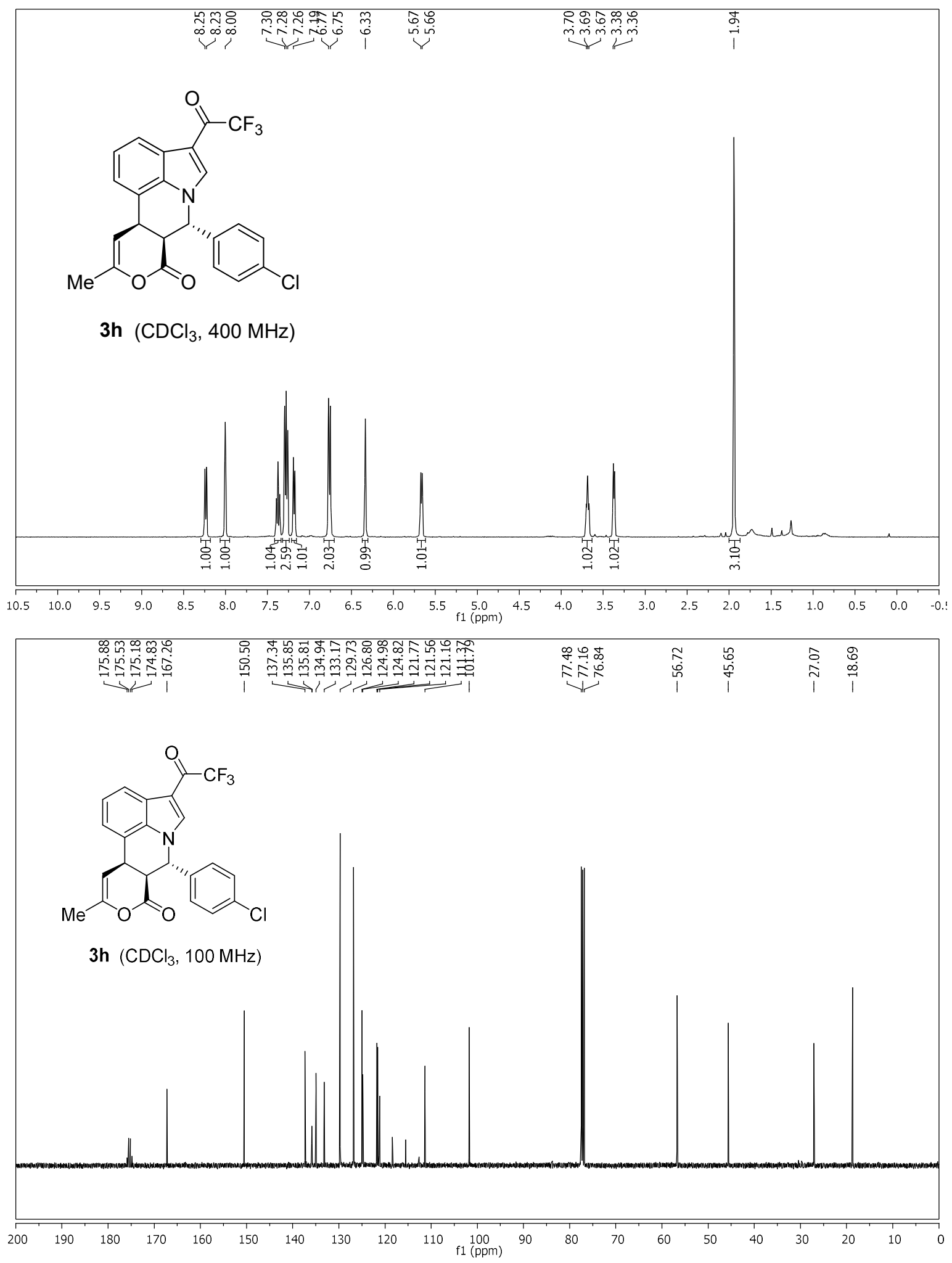

43 
(7S,7aS,11a $R$ )-7-(4-bromophenyl)-10-methyl-4-(2,2,2-trifluoroacetyl)-7a,11a-dihydro7H,8H-pyrano[3,4-c]pyrrolo[3,2,1-ij]quinolin-8-one (3i)
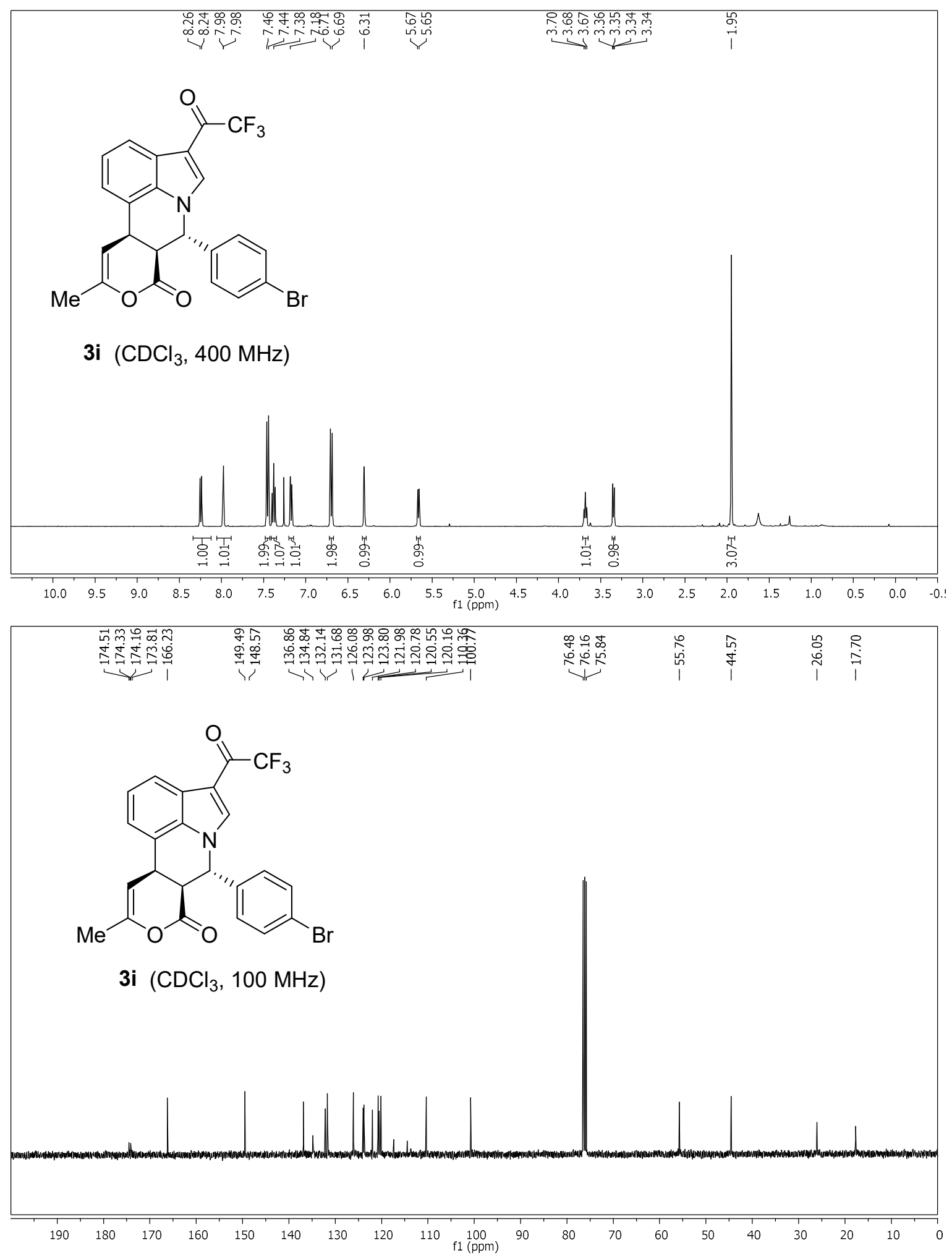
(7S,7aS,11aR)-10-Methyl-4-(2,2,2-trifluoroacetyl)-7-(4-(trifluoromethyl)phenyl)-7,7adihydropyrano[3,4-c]pyrrolo[3,2,1-ij]quinolin-8(11aH)-one (3j)
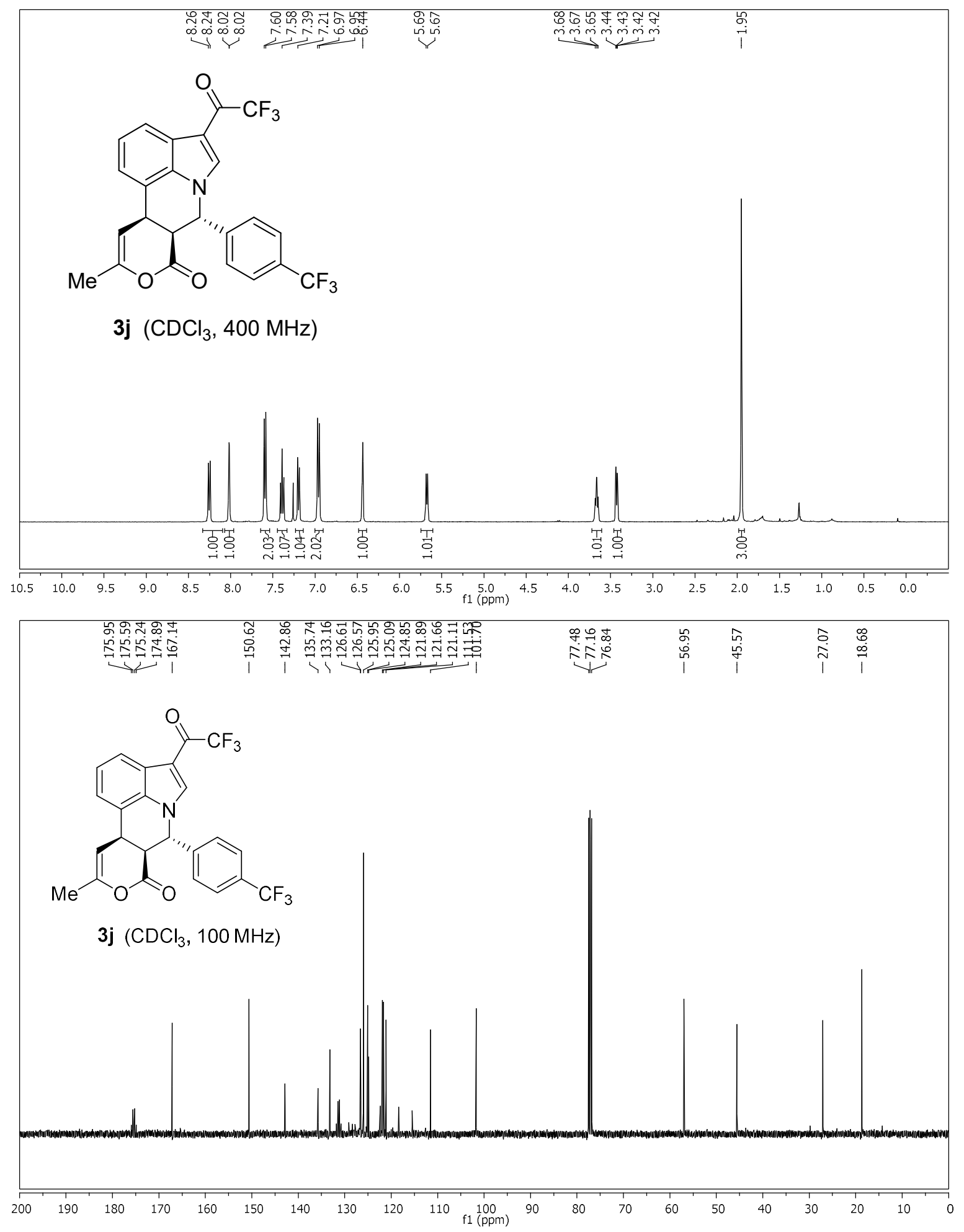
Methyl 4-((7S,7aS,11aR)-10-methyl-8-oxo-4-(2,2,2-trifluoroacetyl)-7a,11a-dihydro-7H, $8 \mathrm{H}$ pyrano[3,4-c]pyrrolo[3,2,1-ij]quinolin-7-yl)benzoate (3k)
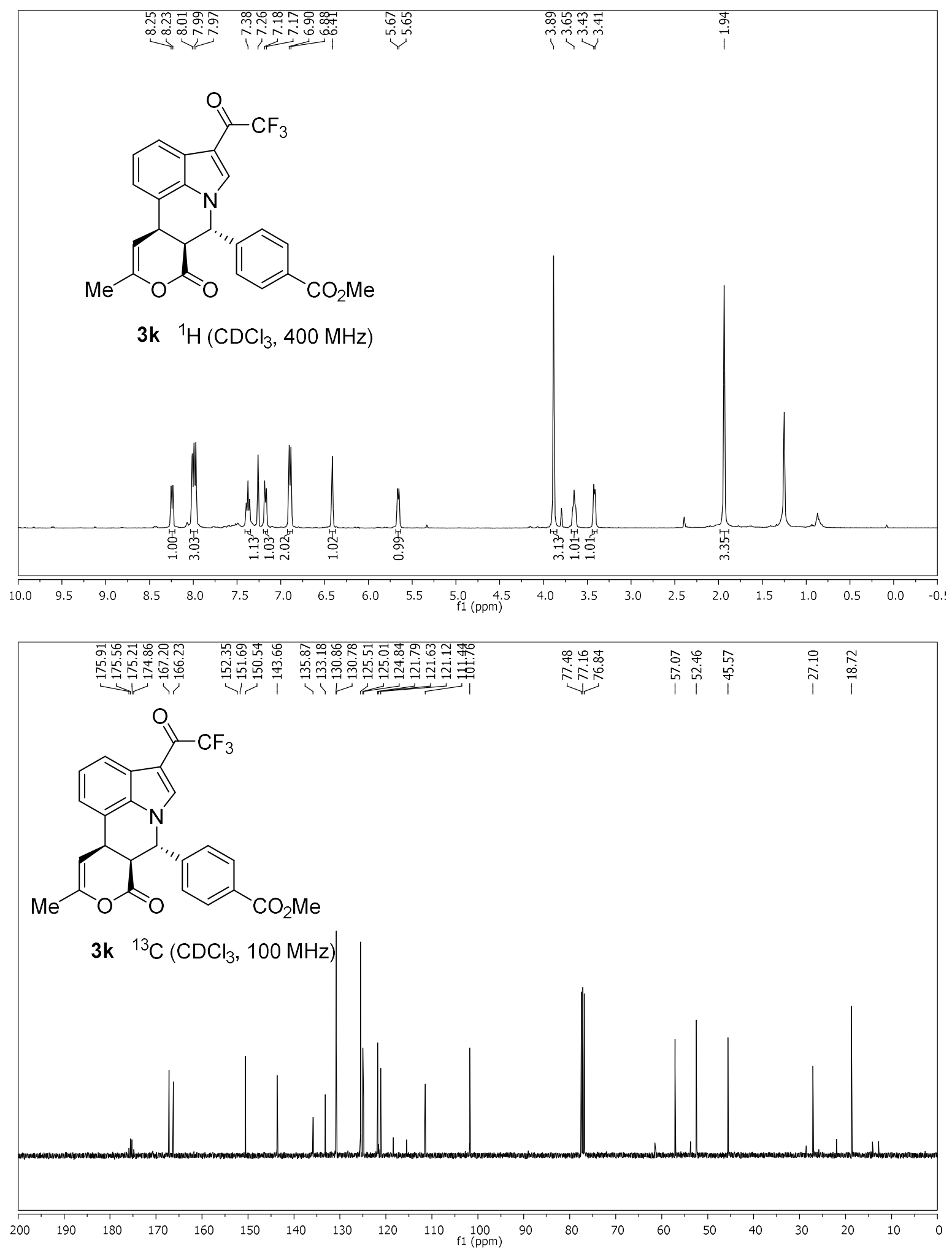
(7S,7aS,11aR)-7-(3-Bromophenyl)-10-methyl-4-(2,2,2-trifluoroacetyl)-7,7adihydropyrano[3,4-c]pyrrolo[3,2,1-ij]quinolin-8(11aH)-one (3I)
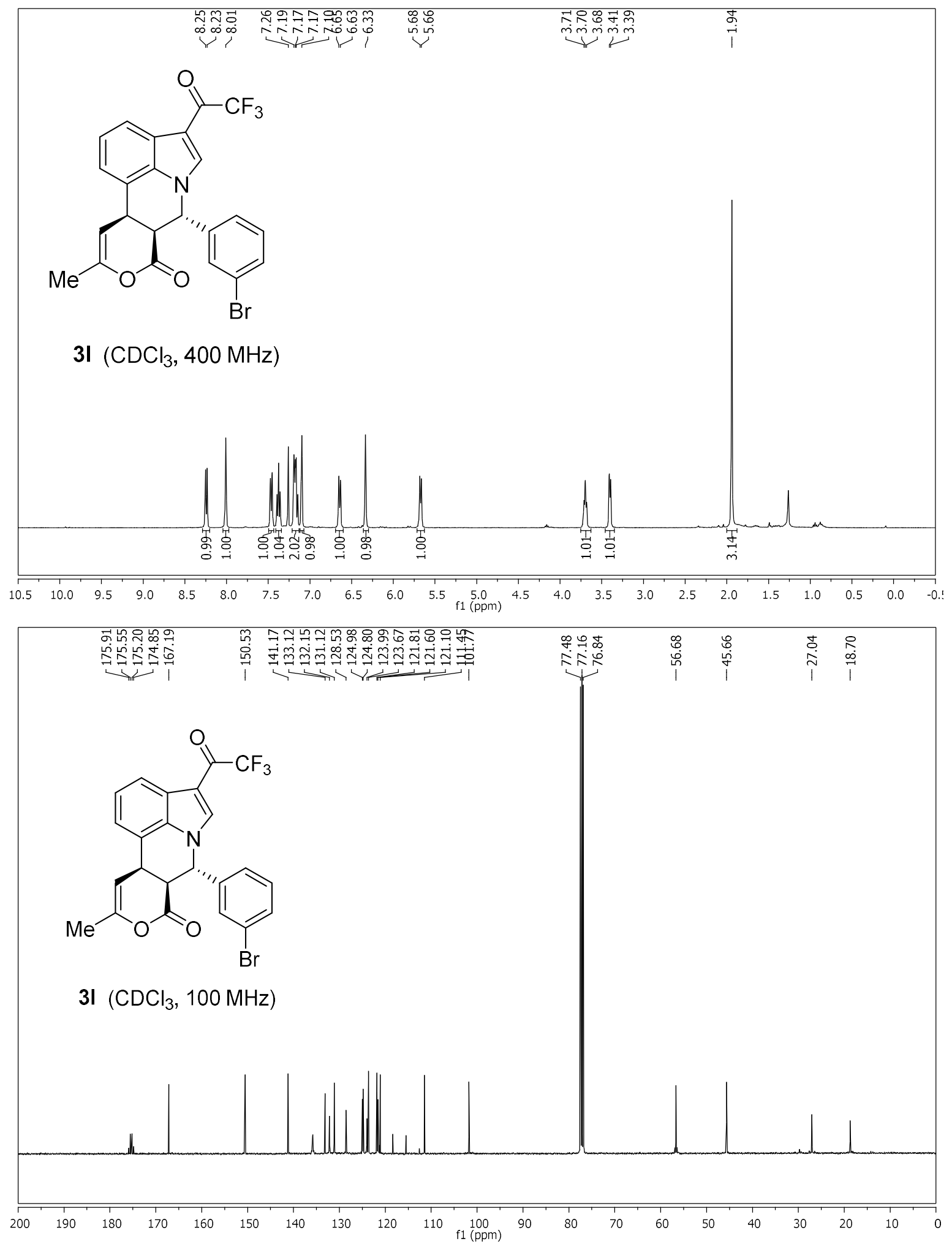
(7S,7aS,11aR)-7-(2-Methoxyphenyl)-10-methyl-4-(2,2,2-trifluoroacetyl)-7a,11a-dihydro7H,8H-pyrano[3,4-c]pyrrolo[3,2,1-ij]quinolin-8-one (3m)
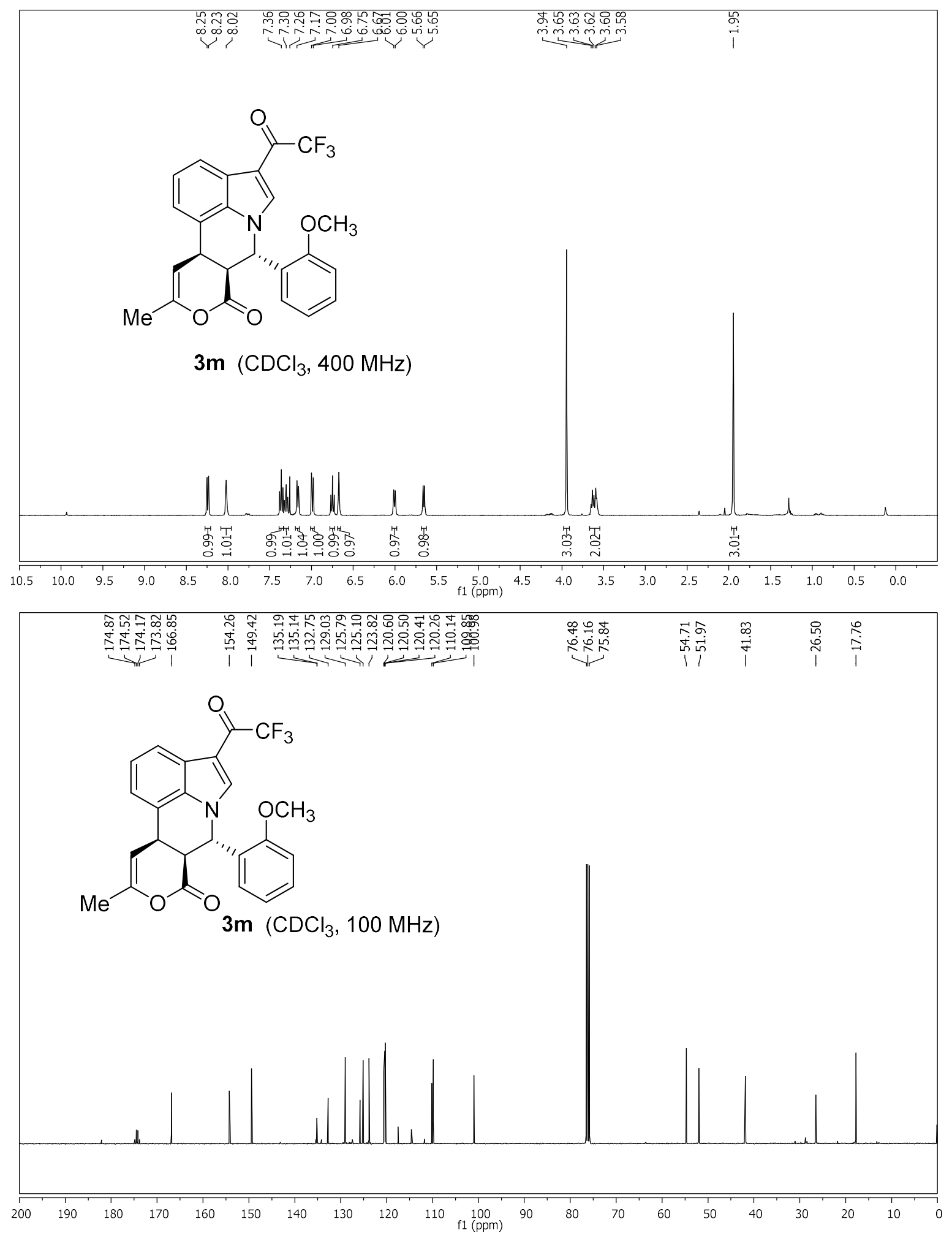
(7S,7aS,11aR)-10-Methyl-7-(o-tolyl)-4-(2,2,2-trifluoroacetyl)-7,7a-dihydropyrano[3,4c]pyrrolo[3,2,1-ij]quinolin-8(11aH)-one (3n)
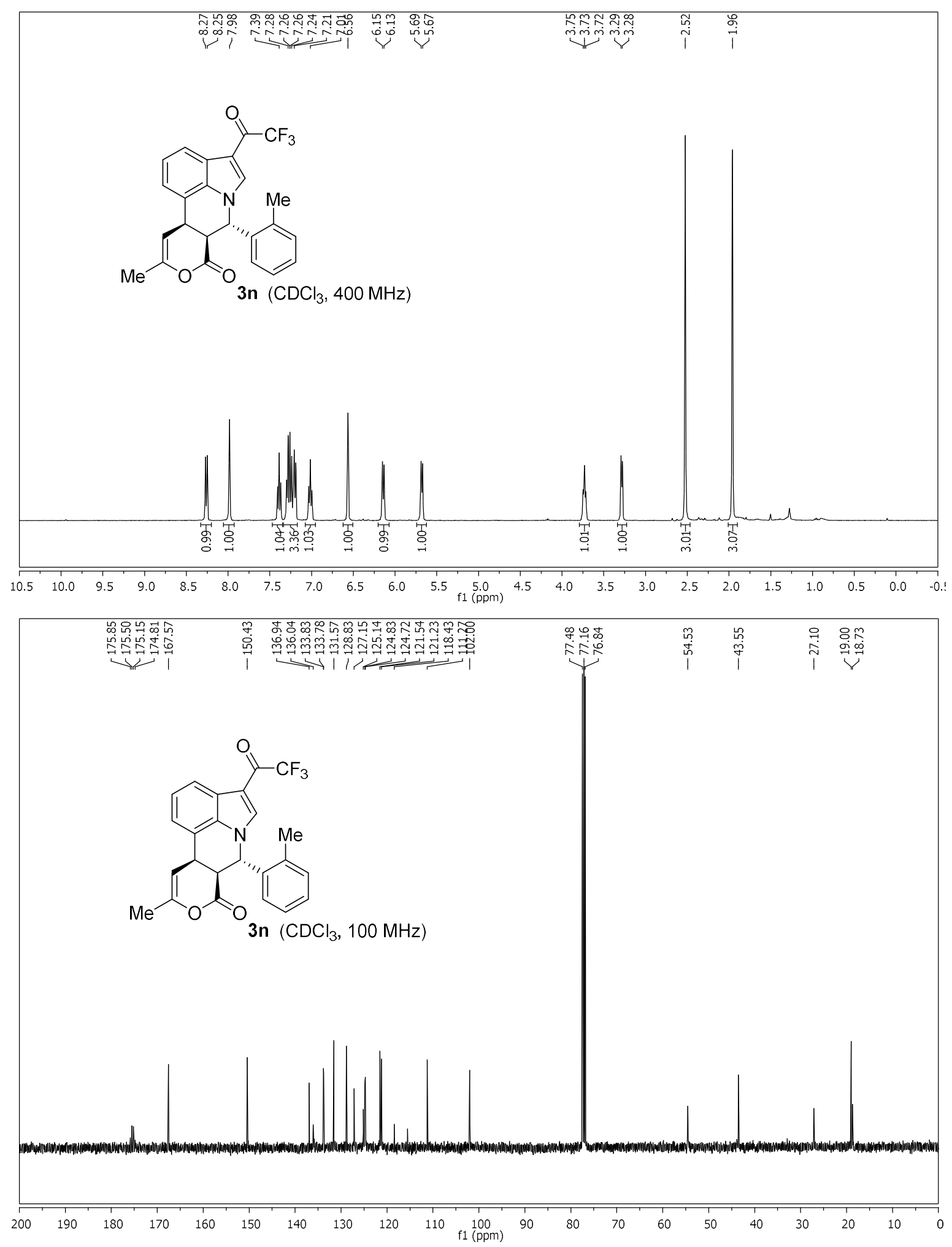
(7S,7aS,11aR)-7-(2-Fluorophenyl)-10-methyl-4-(2,2,2-trifluoroacetyl)-7,7adihydropyrano[3,4-c]pyrrolo[3,2,1-ij]quinolin-8(11aH)-one (3o)
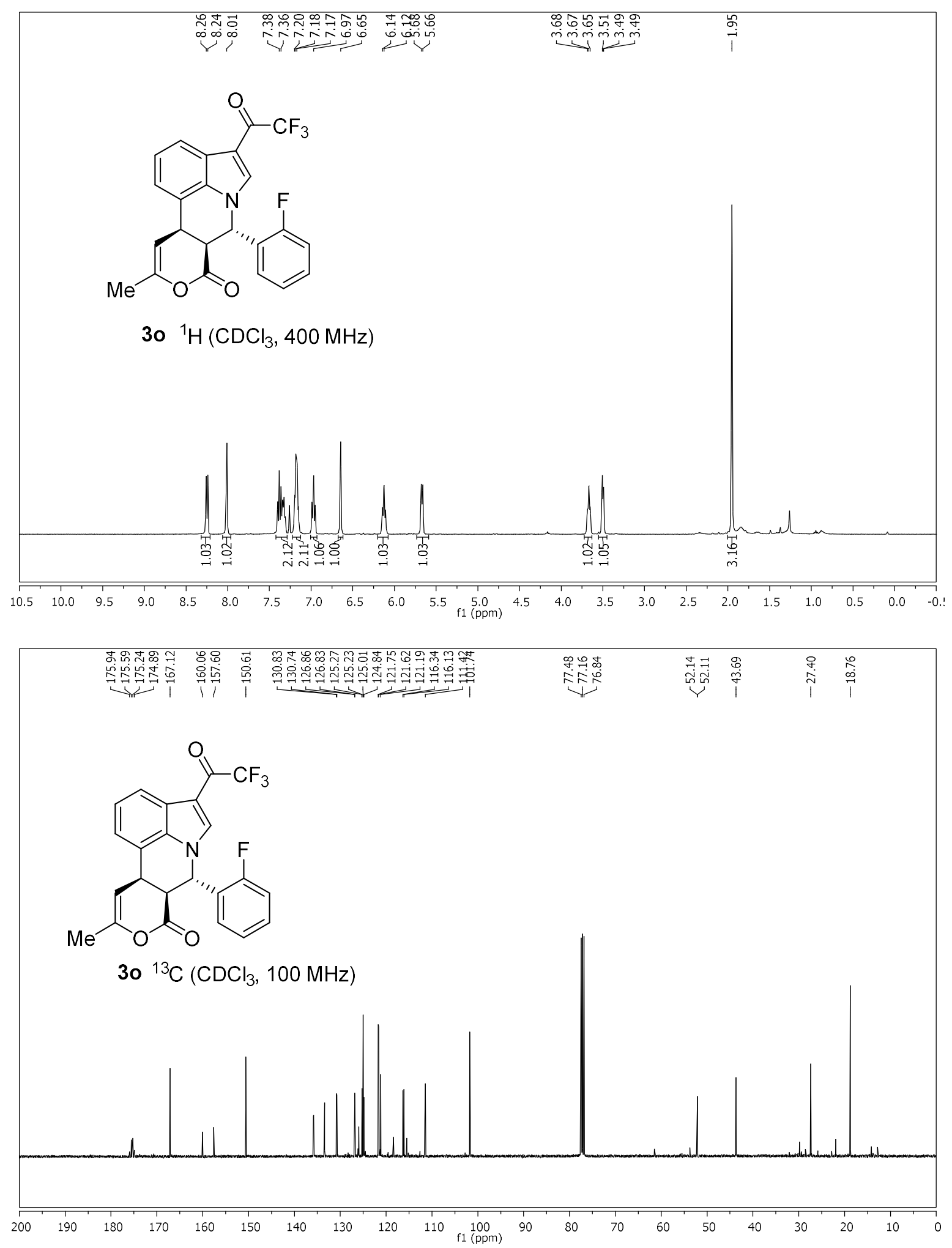
(7S,7aS,11aR)-7-(2-Chlorophenyl)-10-methyl-4-(2,2,2-trifluoroacetyl)-7,7adihydropyrano[3,4-c]pyrrolo[3,2,1-ij]quinolin-8(11aH)-one (3p)
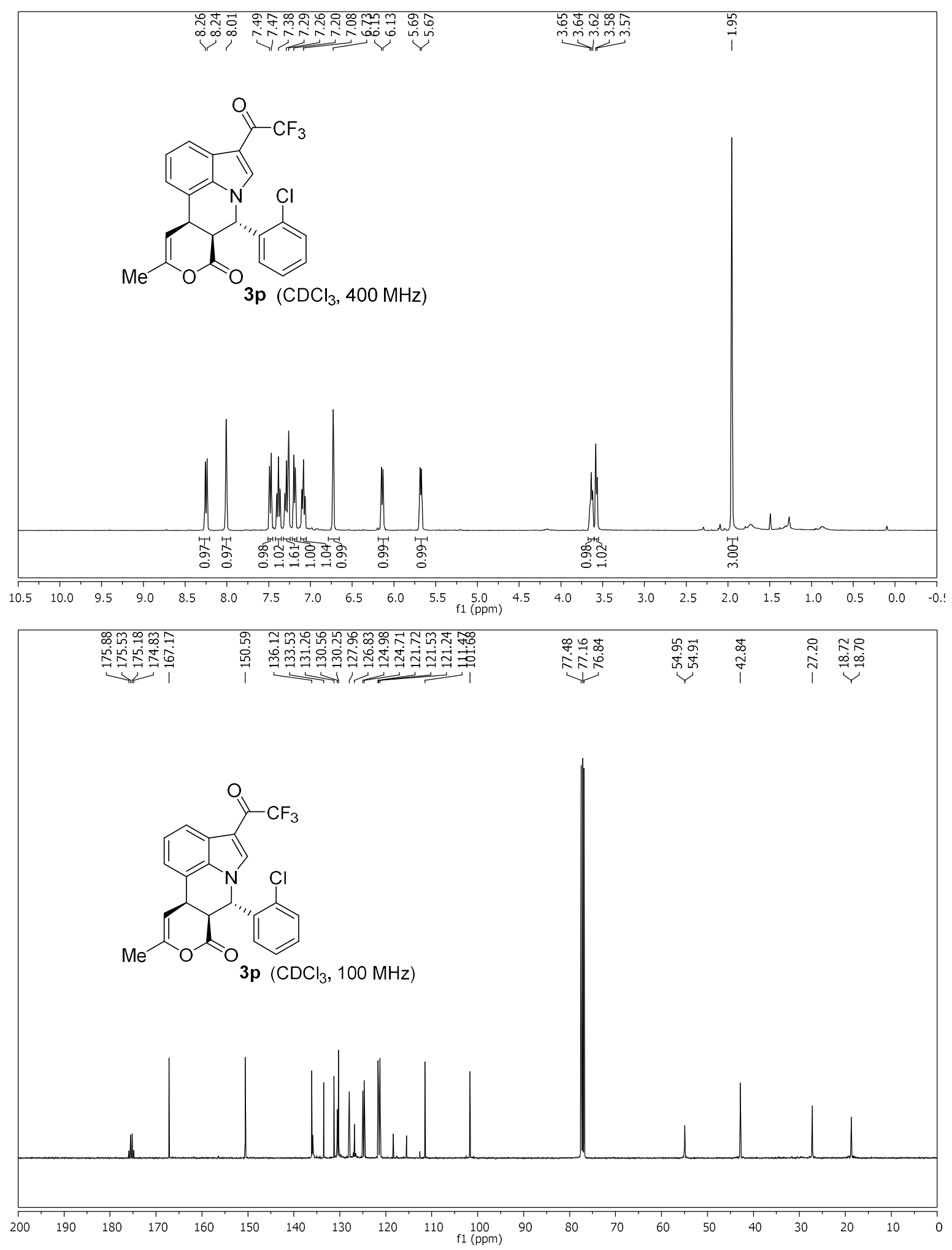
(7S,7aS,11aR)-7-(3,4-Dichlorophenyl)-10-methyl-4-(2,2,2-trifluoroacetyl)-7,7adihydropyrano[3,4-c]pyrrolo[3,2,1-ij]quinolin-8(11aH)-one (3q)

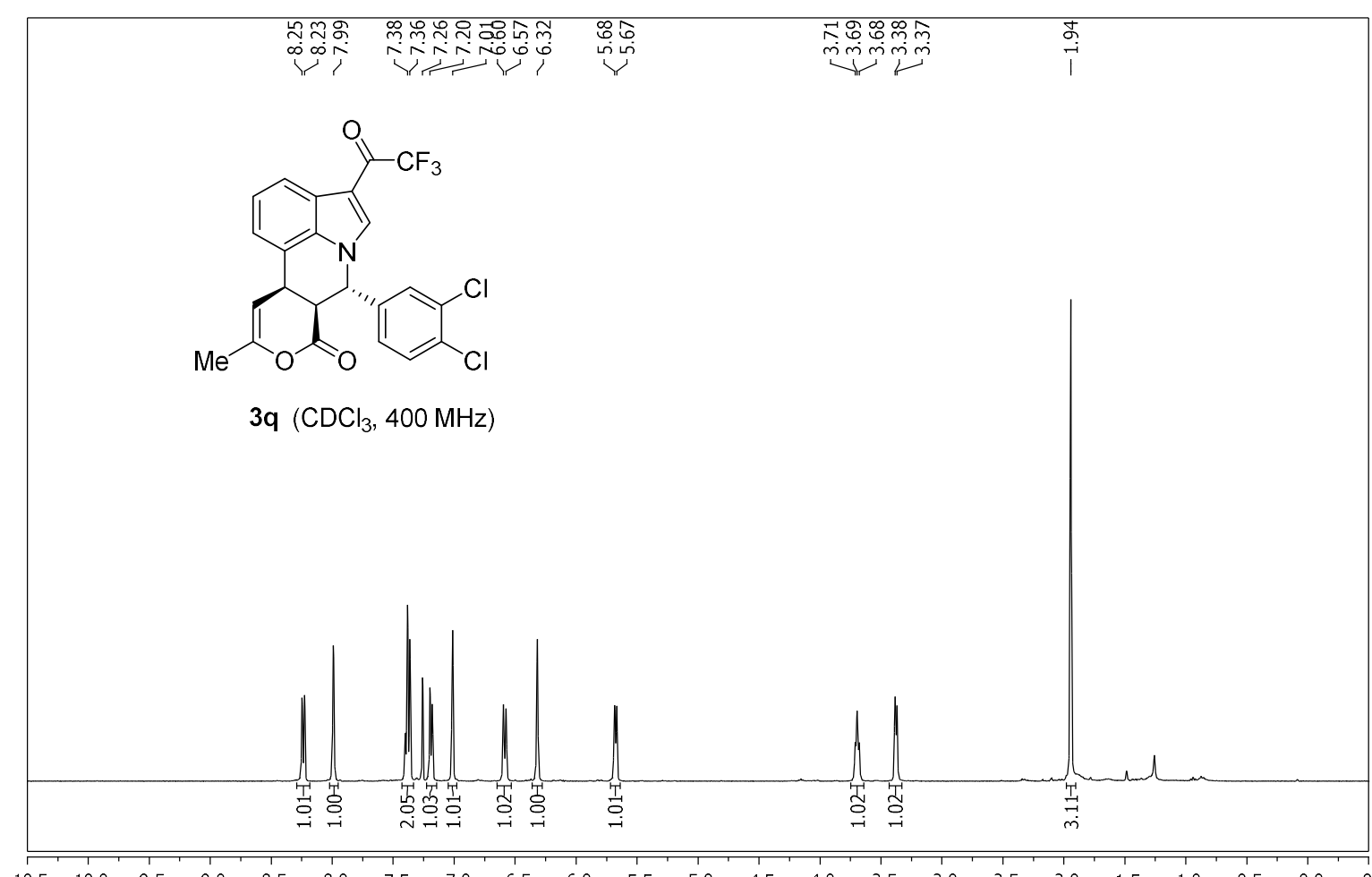

$\begin{array}{llllllllllllllllllllllllll}10.5 & 10.0 & 9.5 & 9.0 & 8.5 & 8.0 & 7.5 & 7.0 & 6.5 & 6.0 & 5.5 & \begin{array}{c}5.0 \\ \mathrm{f} 1\end{array}(\mathrm{pm}) & 4.5 & 4.0 & 3.5 & 3.0 & 2.5 & 2.0 & 1.5 & 1.0 & 0.5 & 0.0 & -0.1\end{array}$

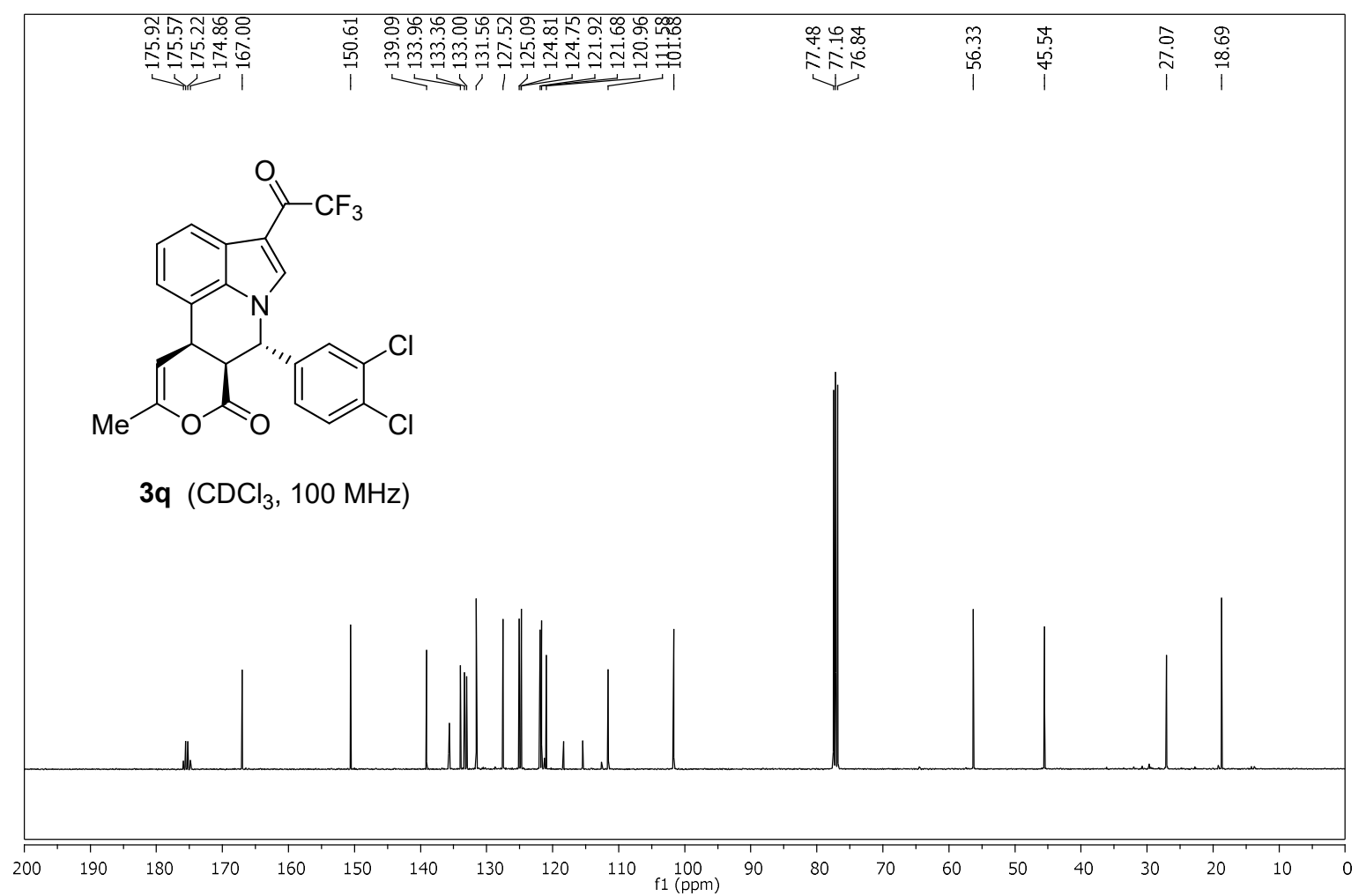


(7S,7aS,11aR)-7-(Benzo[d] [1,3]dioxol-5-yl)-10-methyl-4-(2,2,2-trifluoroacetyl)-7,7adihydropyrano[3,4-c]pyrrolo[3,2,1-ij]quinolin-8(11aH)-one (3r)
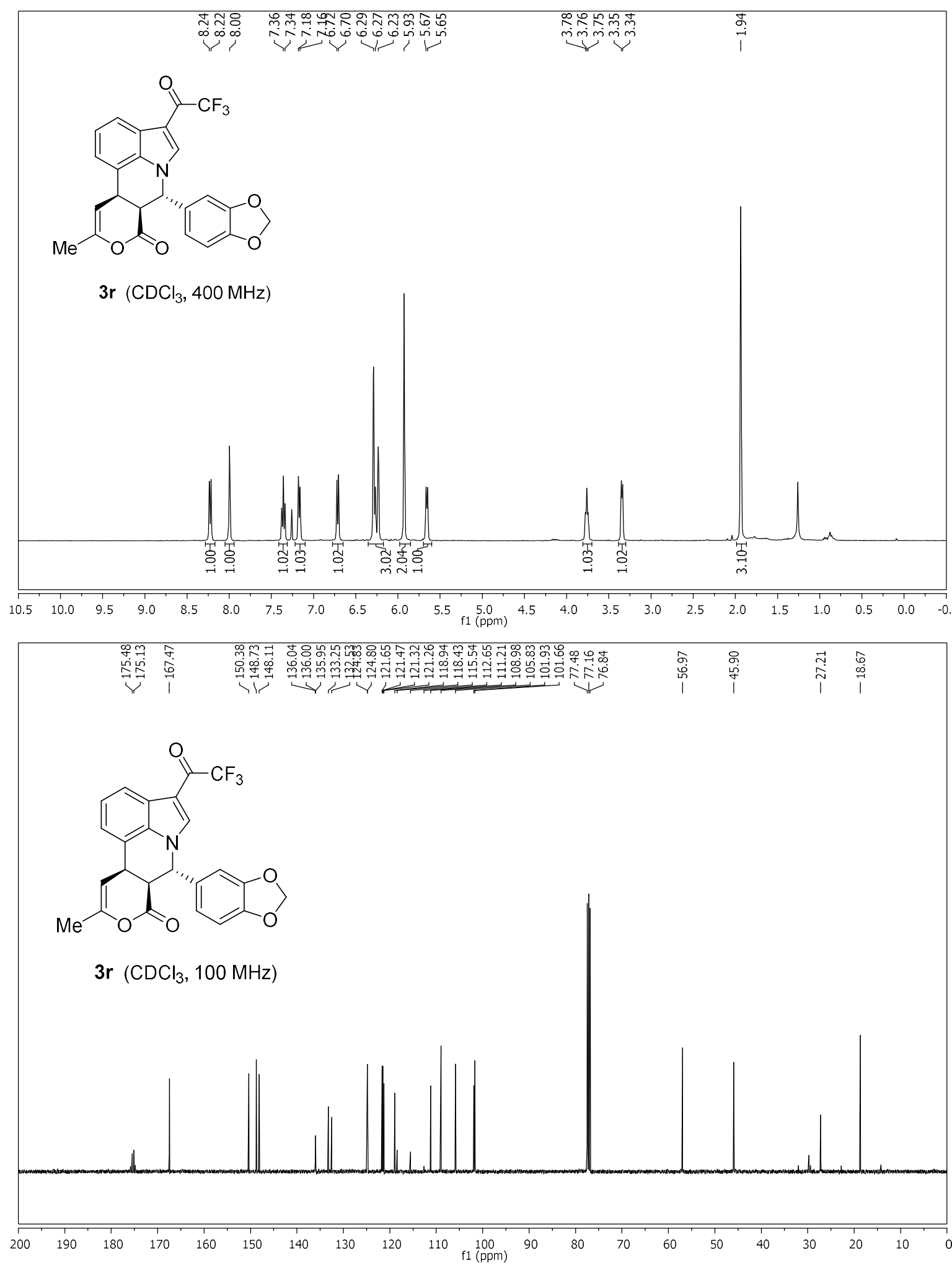
(7S,7aS,11a $R$ )-10-Methyl-7-(naphthalen-2-yl)-4-(2,2,2-trifluoroacetyl)-7a,11 a-dihydro$7 H, 8 H$-pyrano[3,4-c]pyrrolo[3,2,1-ij]quinolin-8-one (3s)
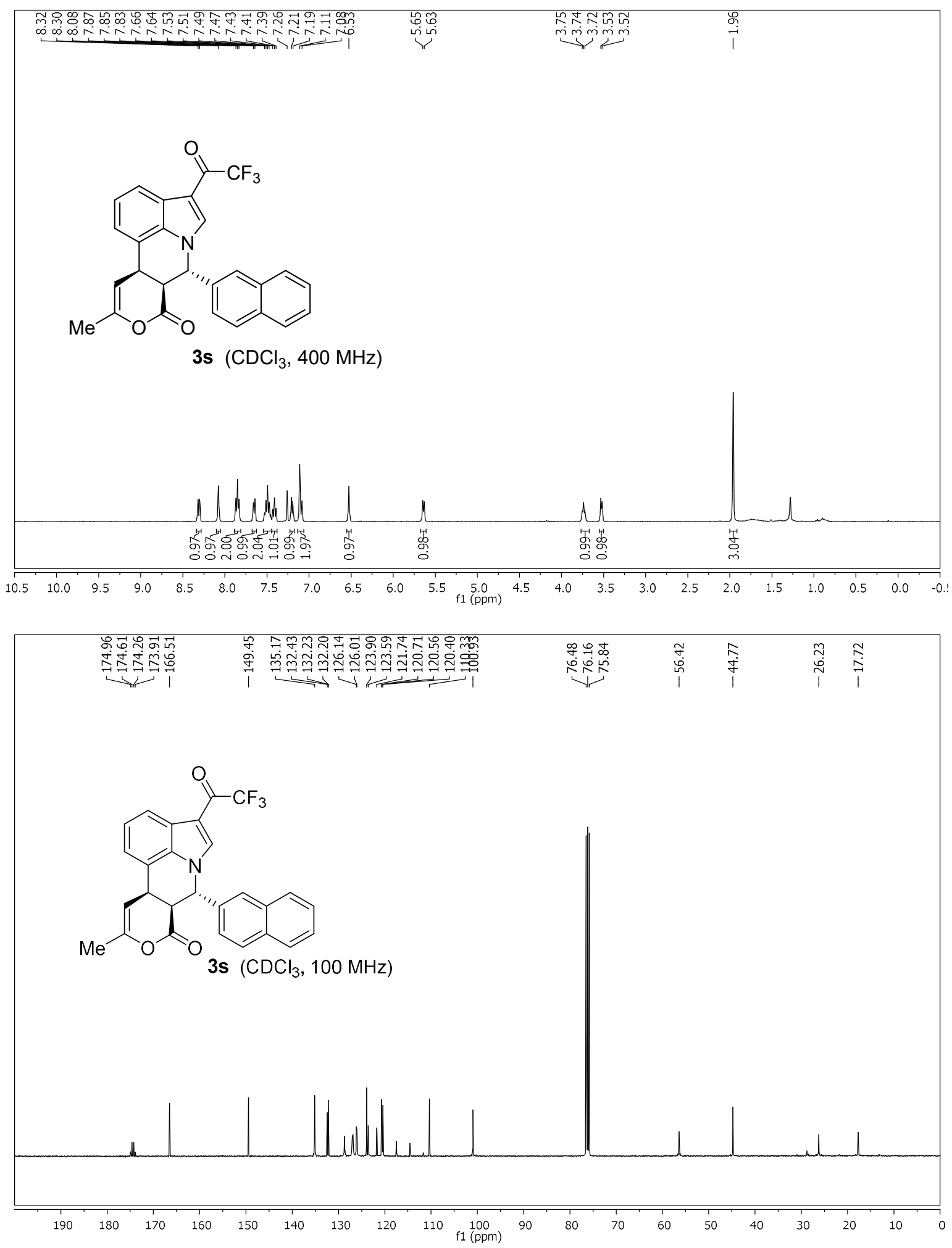

54 
(7S,7aS,11aR)-7-(Furan-2-yl)-10-methyl-4-(2,2,2-trifluoroacetyl)-7,7a-dihydropyrano[3,4c]pyrrolo[3,2,1-ij]quinolin-8(11aH)-one (3t)
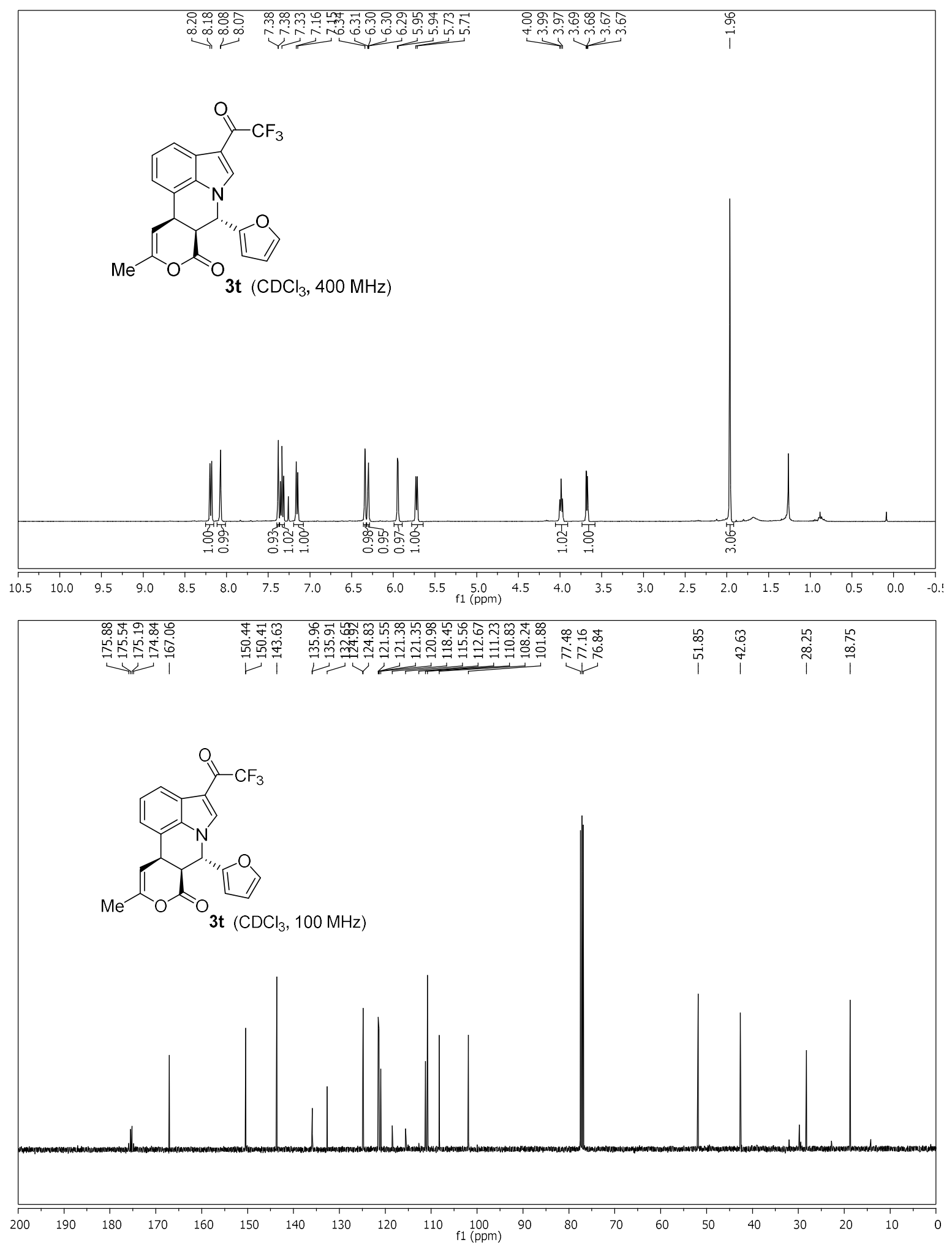
(7S,7aS,11aR)-10-Methyl-7-(thiophen-2-yl)-4-(2,2,2-trifluoroacetyl)-7,7adihydropyrano[3,4-c]pyrrolo[3,2,1-ij]quinolin-8(11aH)-one (3u)
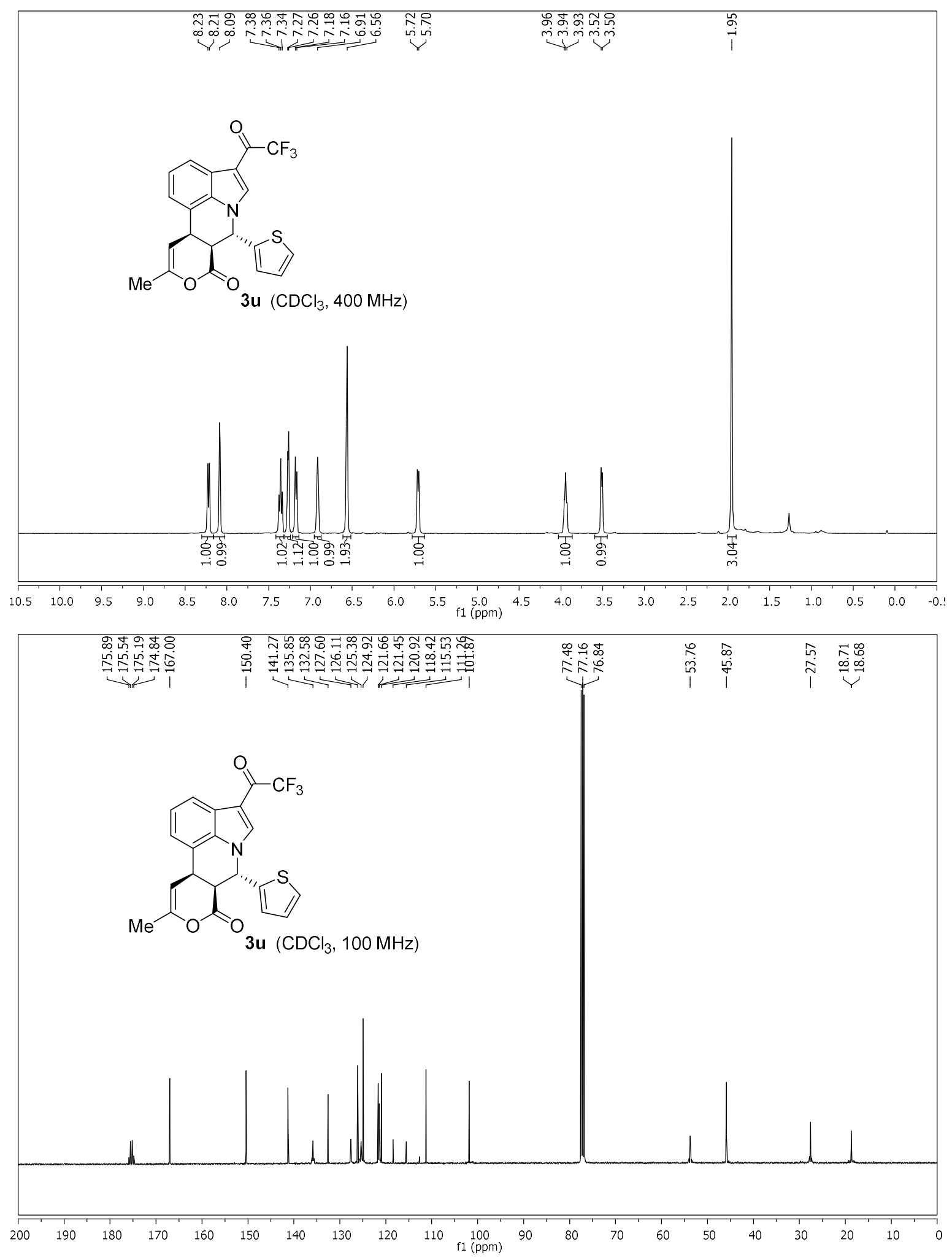
$(7 R, 7 \mathrm{a} S, 11 \mathrm{a} R)-10-M e t h y l-7-((E)-s t y r y l)-4-(2,2,2-t r i f l u o r o a c e t y l)-7 a, 11$ a-dihydro-7H,8Hpyrano[3,4-c]pyrrolo[3,2,1-ij]quinolin-8-one (3v)
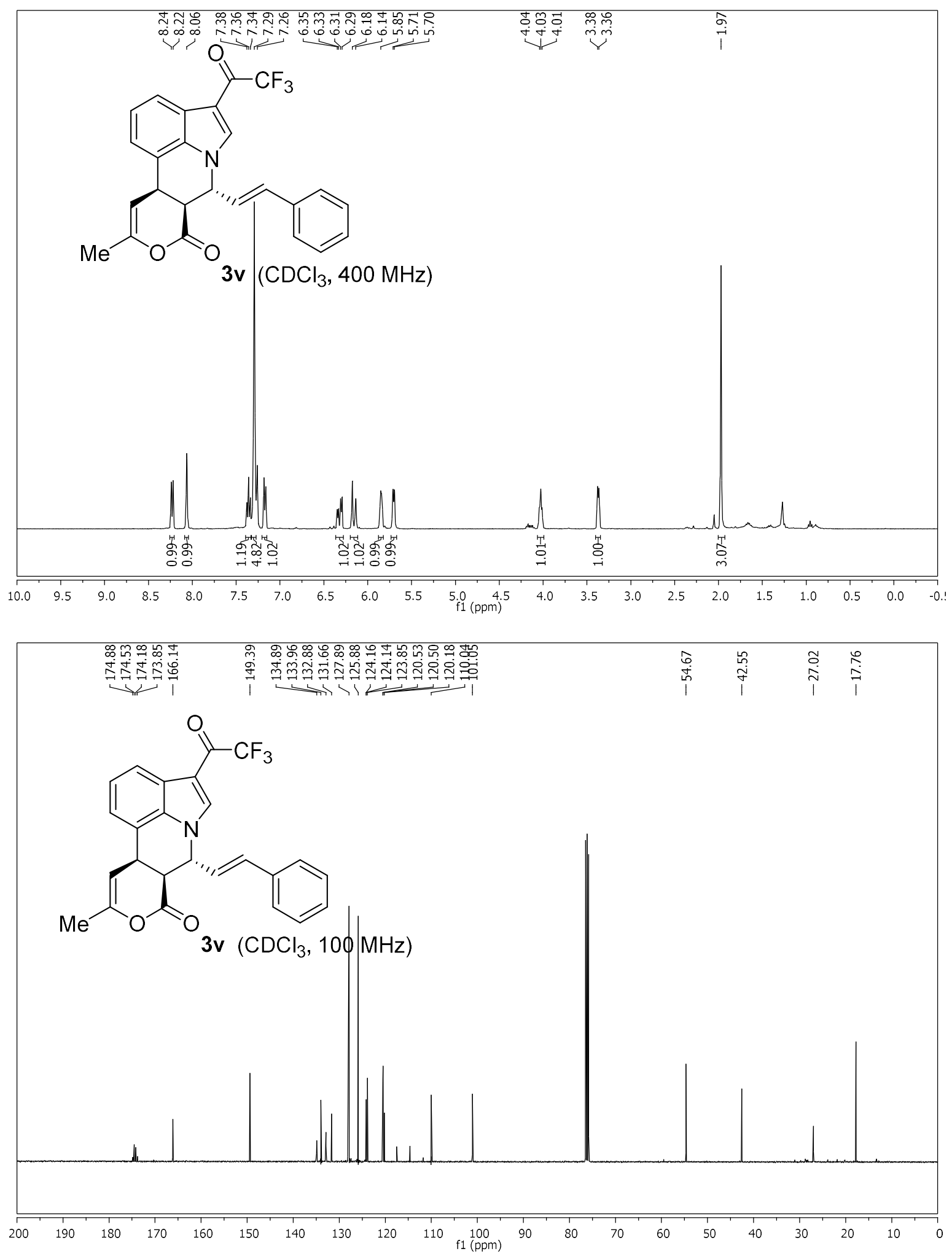
(7S,7aS,11aR)-10-Cyclopropyl-7-phenyl-4-(2,2,2-trifluoroacetyl)-7,7a-dihydropyrano[3,4c]pyrrolo[3,2,1-ij]quinolin-8(11aH)-one (3w)
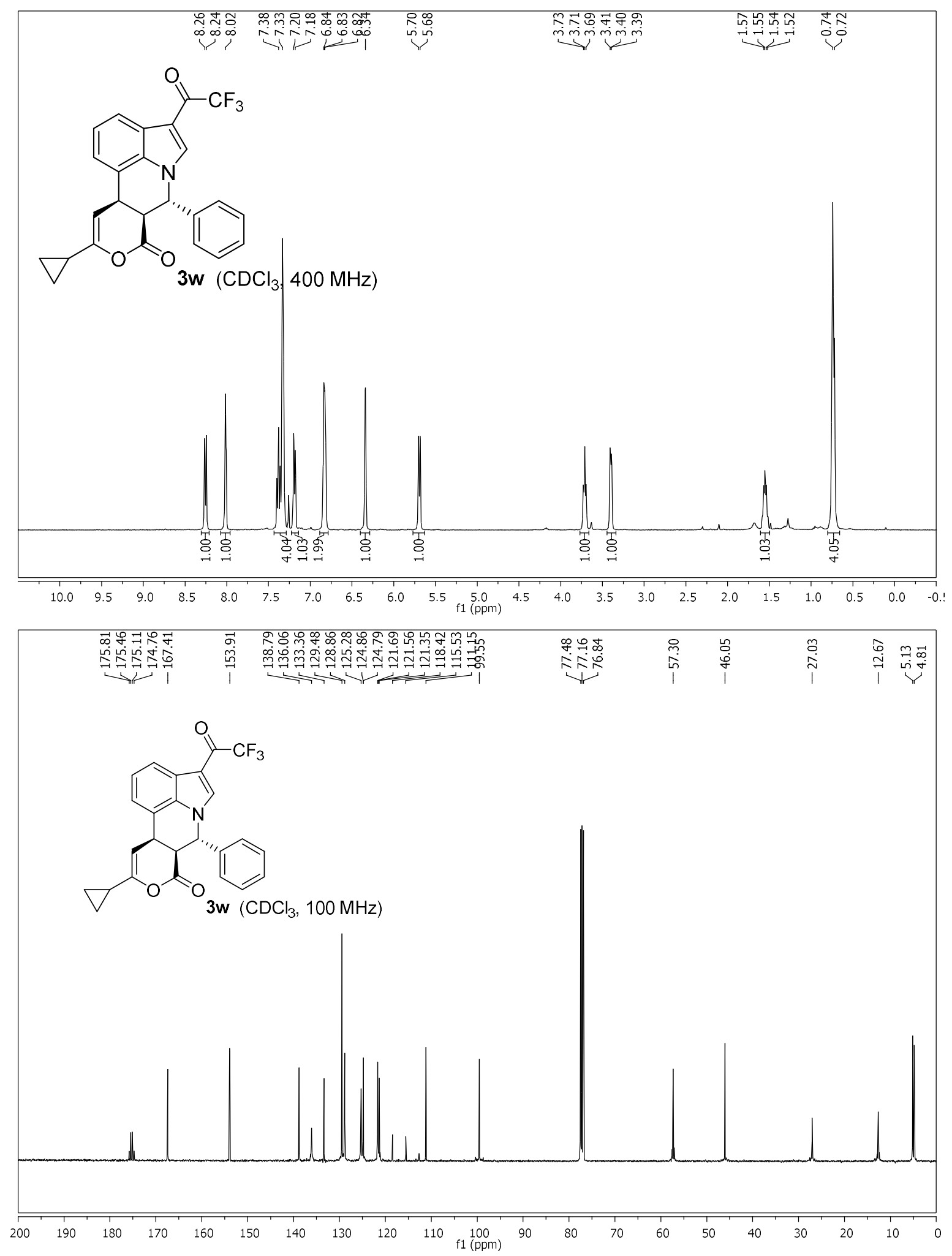
(7S,7aS,11a R)-10-Isobutyl-7-phenyl-4-(2,2,2-trifluoroacetyl)-7,7a-dihydropyrano[3,4c]pyrrolo[3,2,1-ij]quinolin-8(11aH)-one (3x)
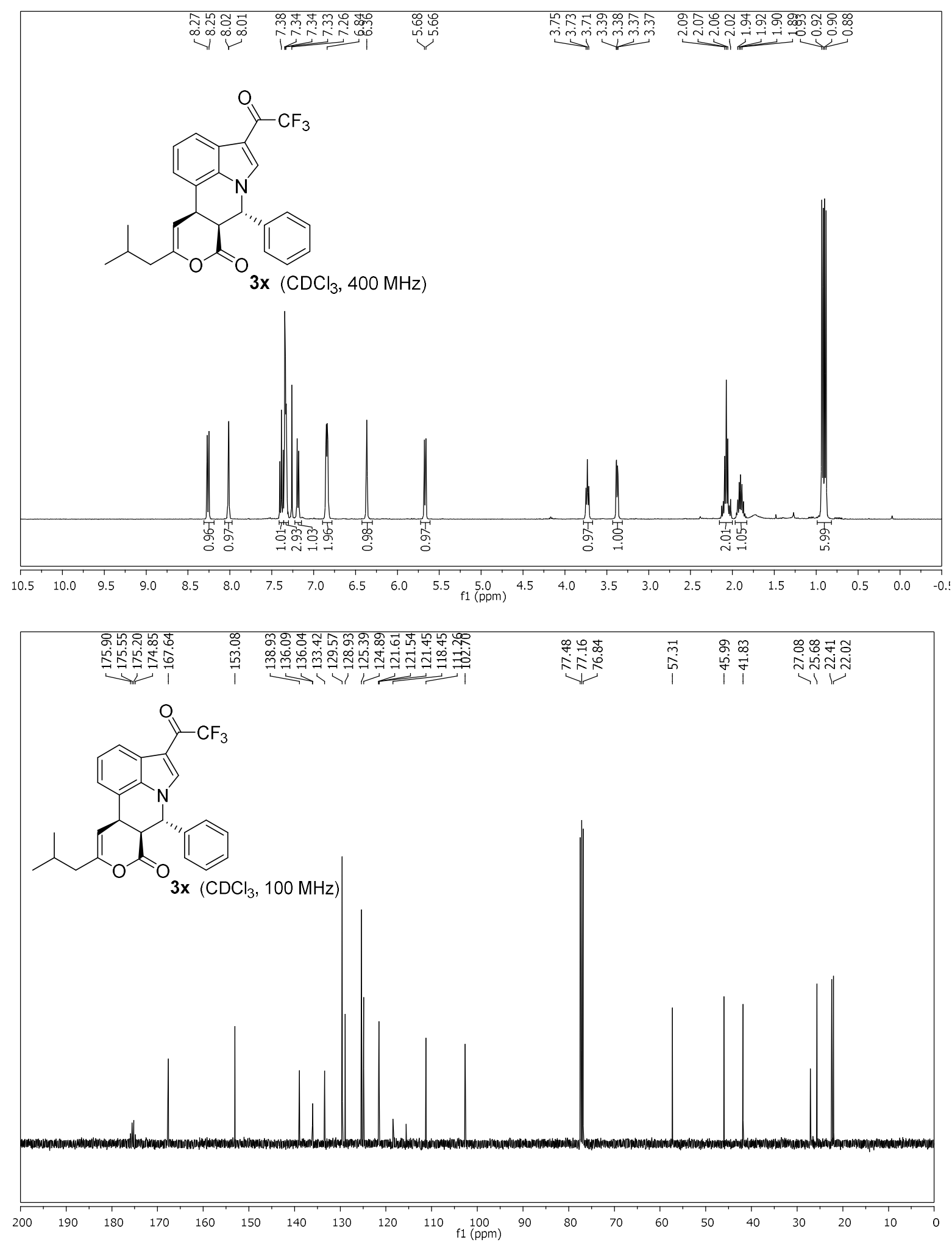
(7S,7aS,11a $R$ )-2-Bromo-10-methyl-7-phenyl-4-(2,2,2-trifluoroacetyl)-7,7adihydropyrano[3,4-c]pyrrolo[3,2,1-ij]quinolin-8(11aH)-one (3y)
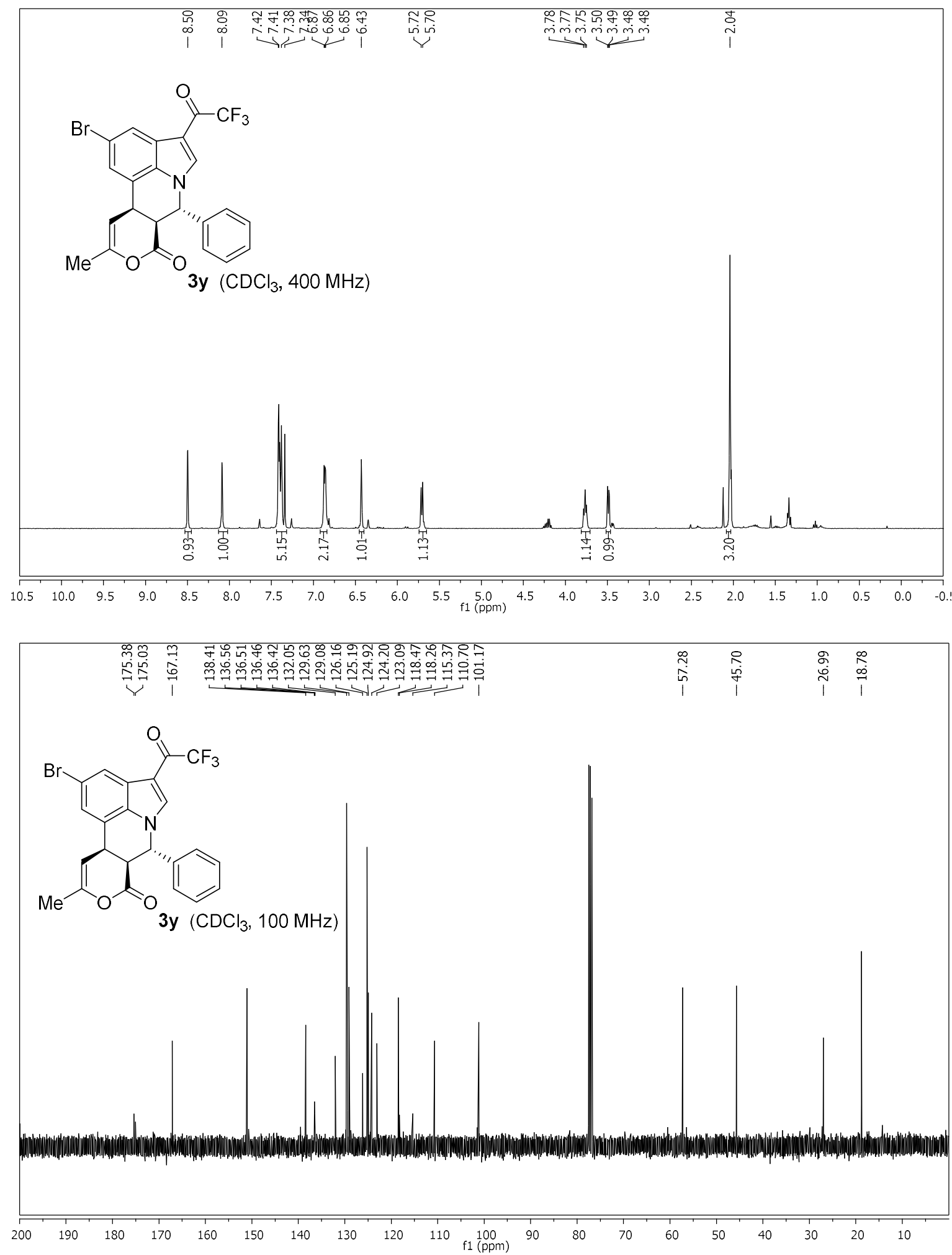
(4S,5S,6R)-Methyl 6-(2-oxopropyl)-4-phenyl-1-(2,2,2-trifluoroacetyl)-5,6-dihydro-4Hpyrrolo[3,2,1-ij]quinoline-5-carboxylate (6d)
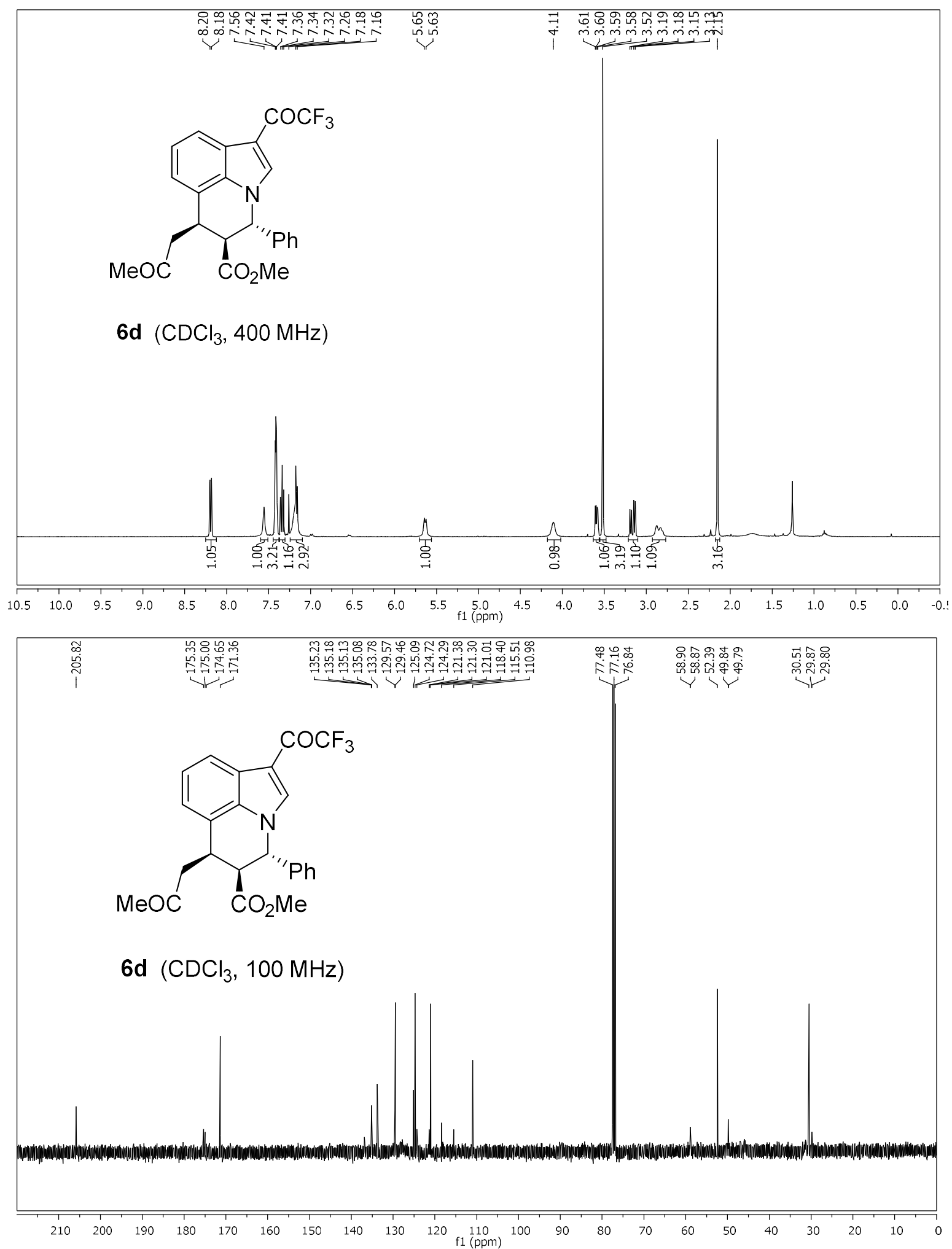
(4S,5S,6R)-N-Benzyl-6-(2-oxopropyl)-4-phenyl-1-(2,2,2-trifluoroacetyl)-5,6-dihydro-4Hpyrrolo[3,2,1-ij]quinoline-5-carboxamide (7d)
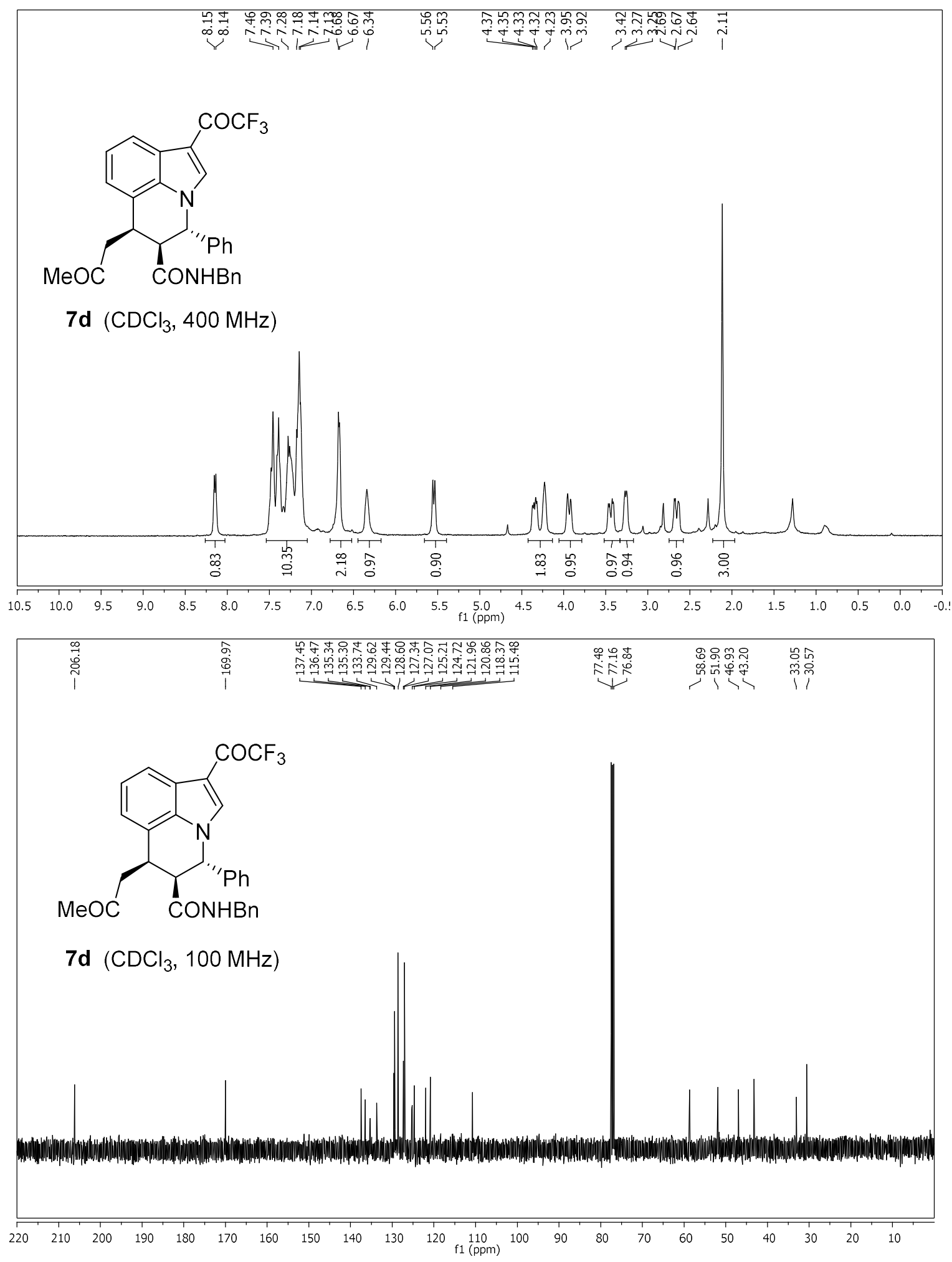
(E)-Methyl 3-((7S,7aS,11aR)-10-methyl-8-oxo-7-phenyl-4-(2,2,2-trifluoroacetyl)-7,7a,8,11atetrahydropyrano[3,4-c]pyrrolo[3,2,1-ij]quinolin-3-yl)acrylate (8d)
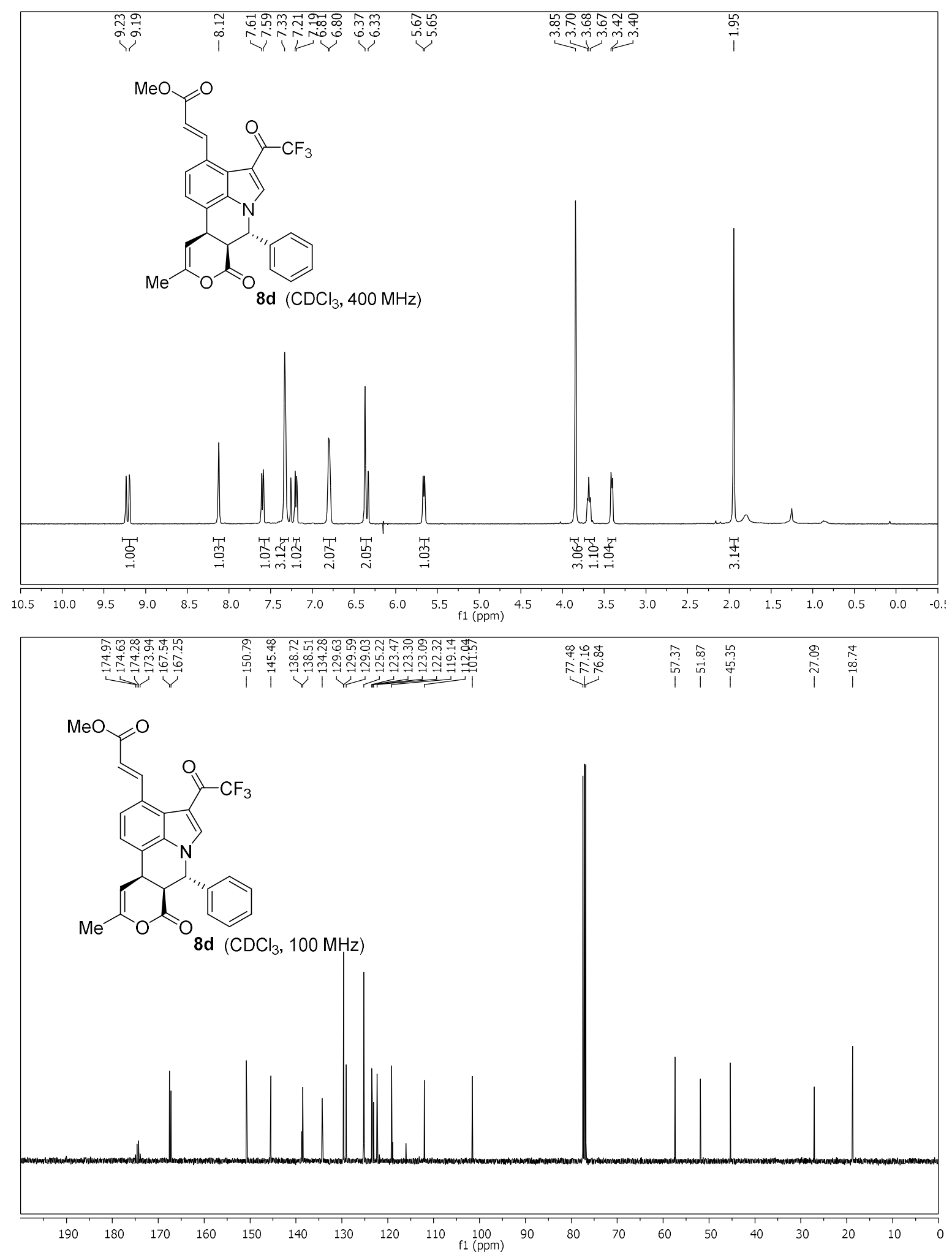
1-Benzyl-3-((7S,7aS,11aR)-10-methyl-8-oxo-7-phenyl-4-(2,2,2-trifluoroacetyl)7,7a,8,11atetrahydropyrano[3,4-c]pyrrolo[3,2,1-ij]quinolin-3-yl)pyrrolidine-2,5-dione (9d)
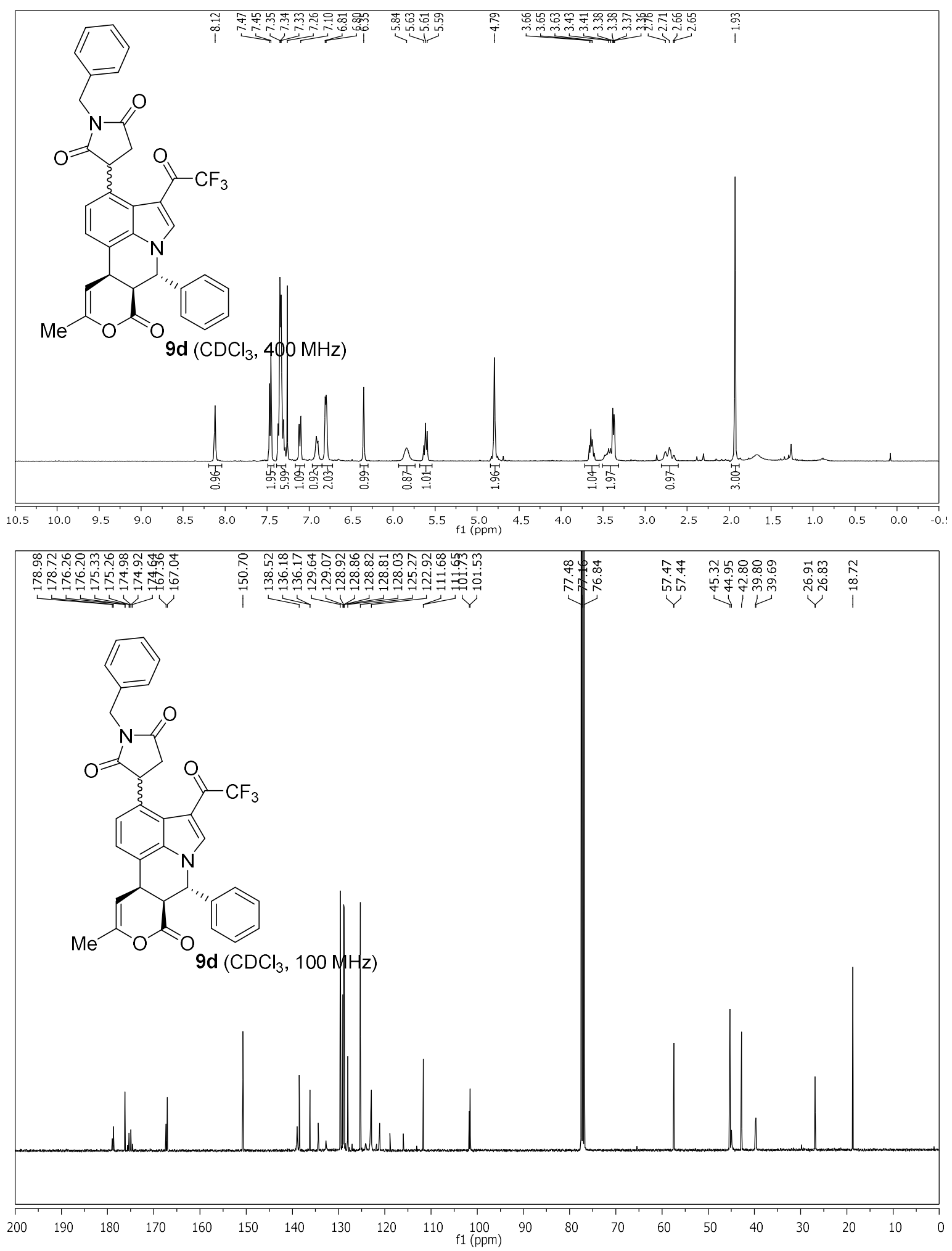

64 


\section{HPLC data of Pyrroloquinoline Derivatives}

(7S,7aS,11a R)-10-methyl-8-oxo-7-phenyl-7a,11a-dihydro-7H,8H-pyrano[3,4c]pyrrolo[3,2,1-ij]quinoline-4-carbaldehyde (3c)
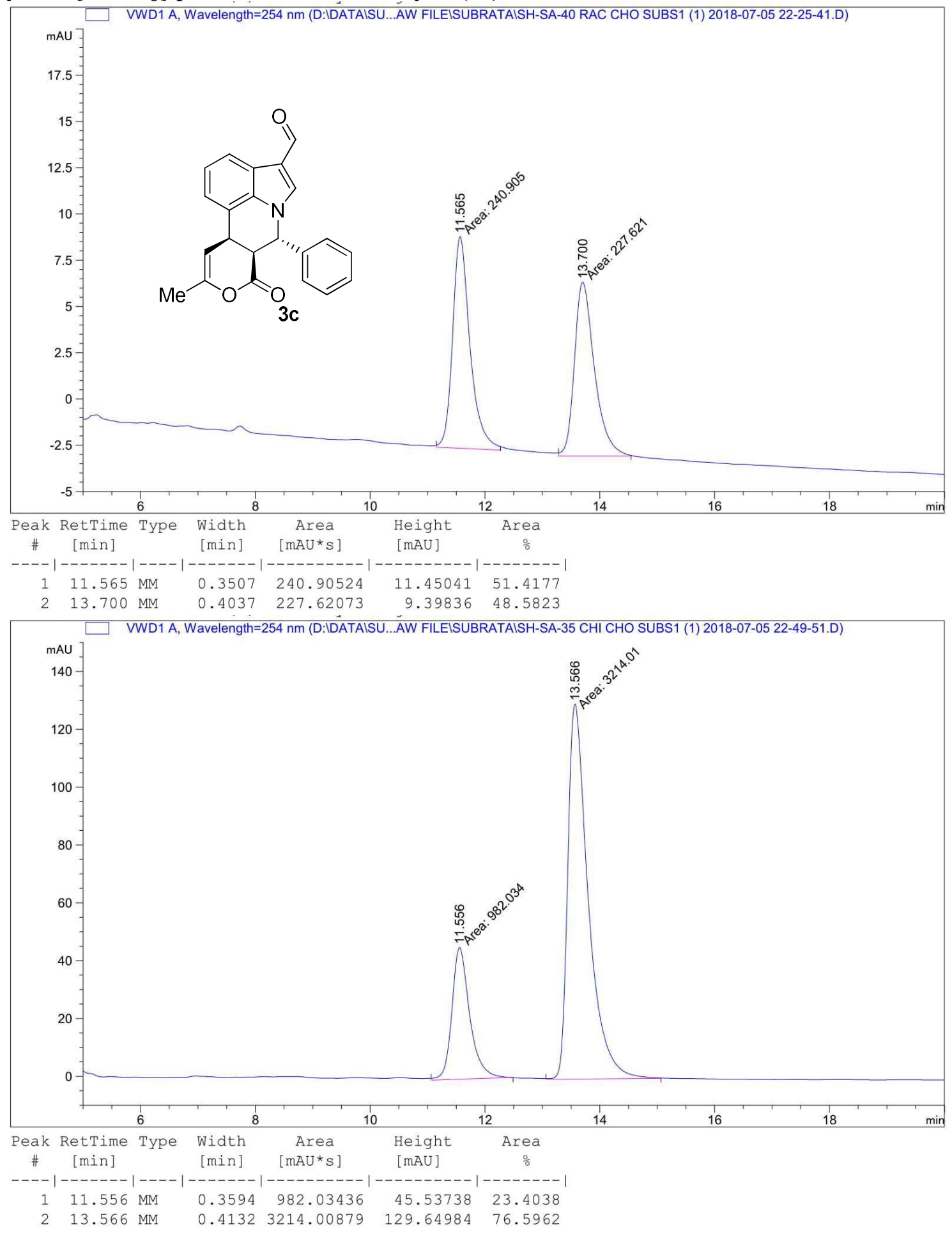

Sample Info : CHIRALPAK-AD, $20 \%$ IPA-Hexane, $1.0 \mathrm{ml} / \mathrm{min}, 254 \mathrm{~nm}$ 
(7S,7aS,11aR)-10-Methyl-7-phenyl-4-(2,2,2-trifluoroacetyl)-7,7a-dihydropyrano[3,4c]pyrrolo[3,2,1-ij]quinolin-8(11aH)-one (3d)
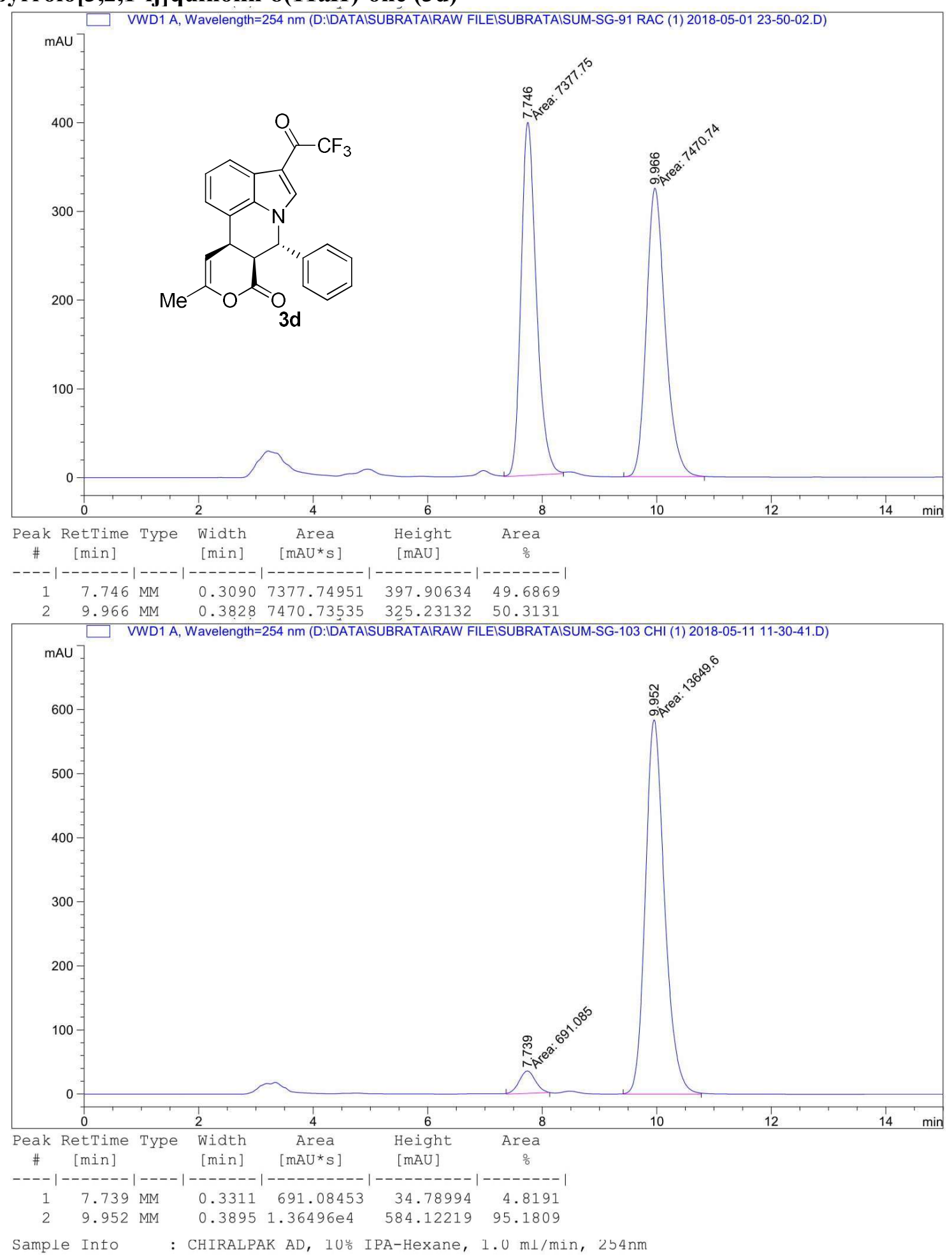
(7S,7aS,11aR)-7-(4-(Dimethylamino)phenyl)-10-methyl-4-(2,2,2-trifluoroacetyl)-7,7adihydropyrano[3,4-c]pyrrolo[3,2,1-ij]quinolin-8(11aH)-one (3e)

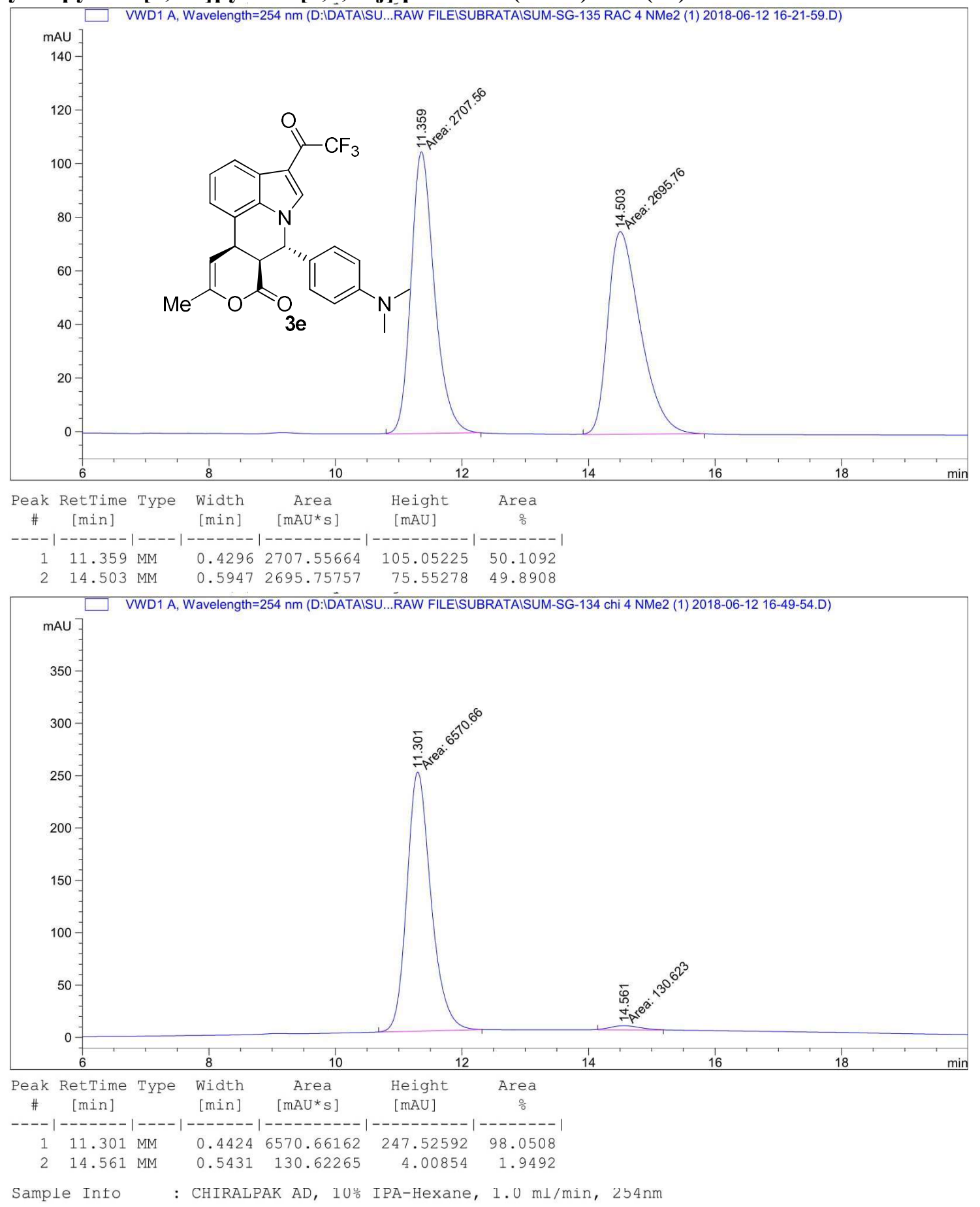

67 
(7S,7aS,11aR)-7-(4-Methoxyphenyl)-10-methyl-4-(2,2,2-trifluoroacetyl)-7,7adihydropyrano[3,4-c]pyrrolo[3,2,1-ij]quinolin-8(11aH)-one (3f)

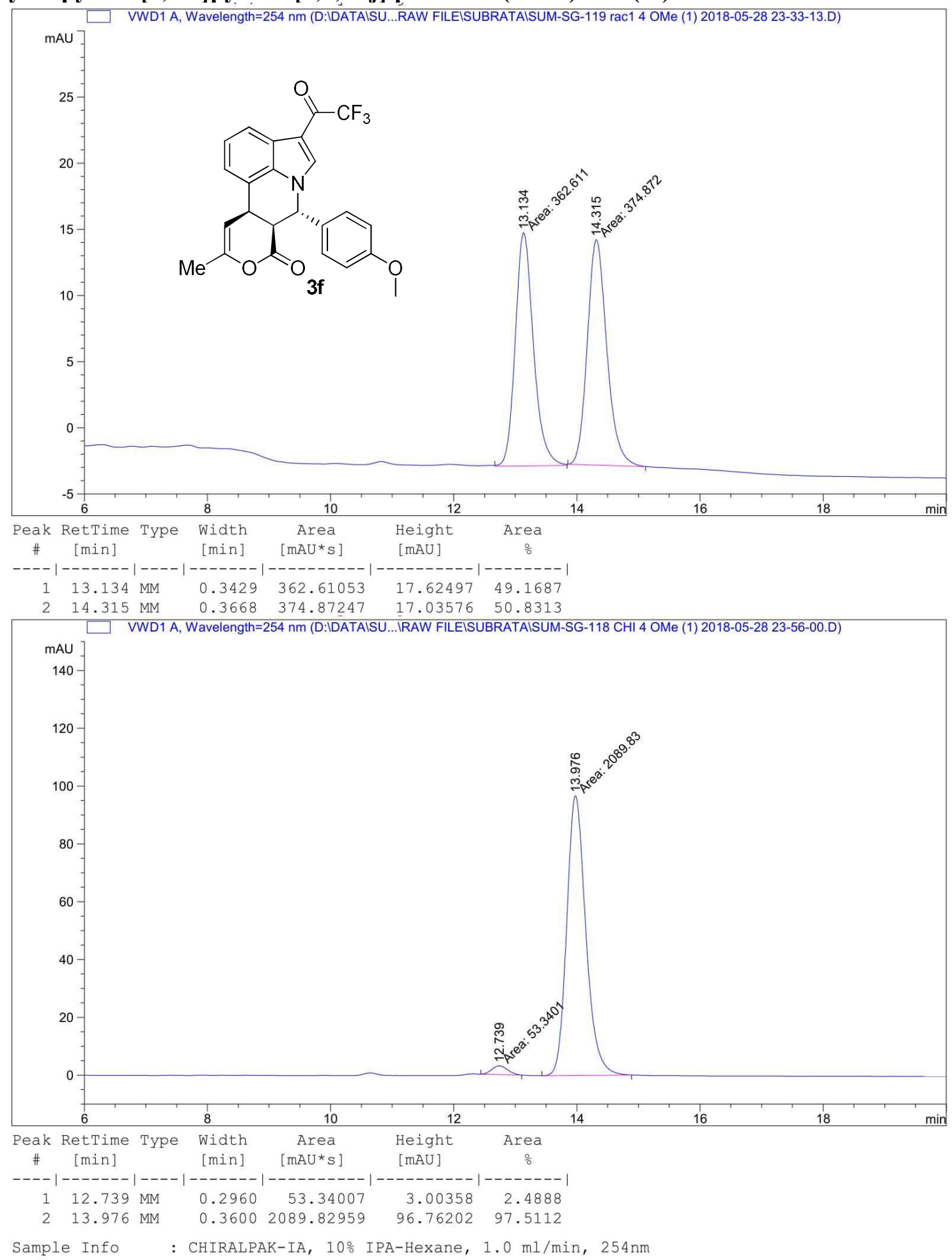

68 
(7S,7aS,11aR)-10-Methyl-7-(p-tolyl)-4-(2,2,2-trifluoroacetyl)-7a,11a-dihydro-7H,8Hpyrano[3,4-c]pyrrolo[3,2,1-ij]quinolin-8-one (3g)
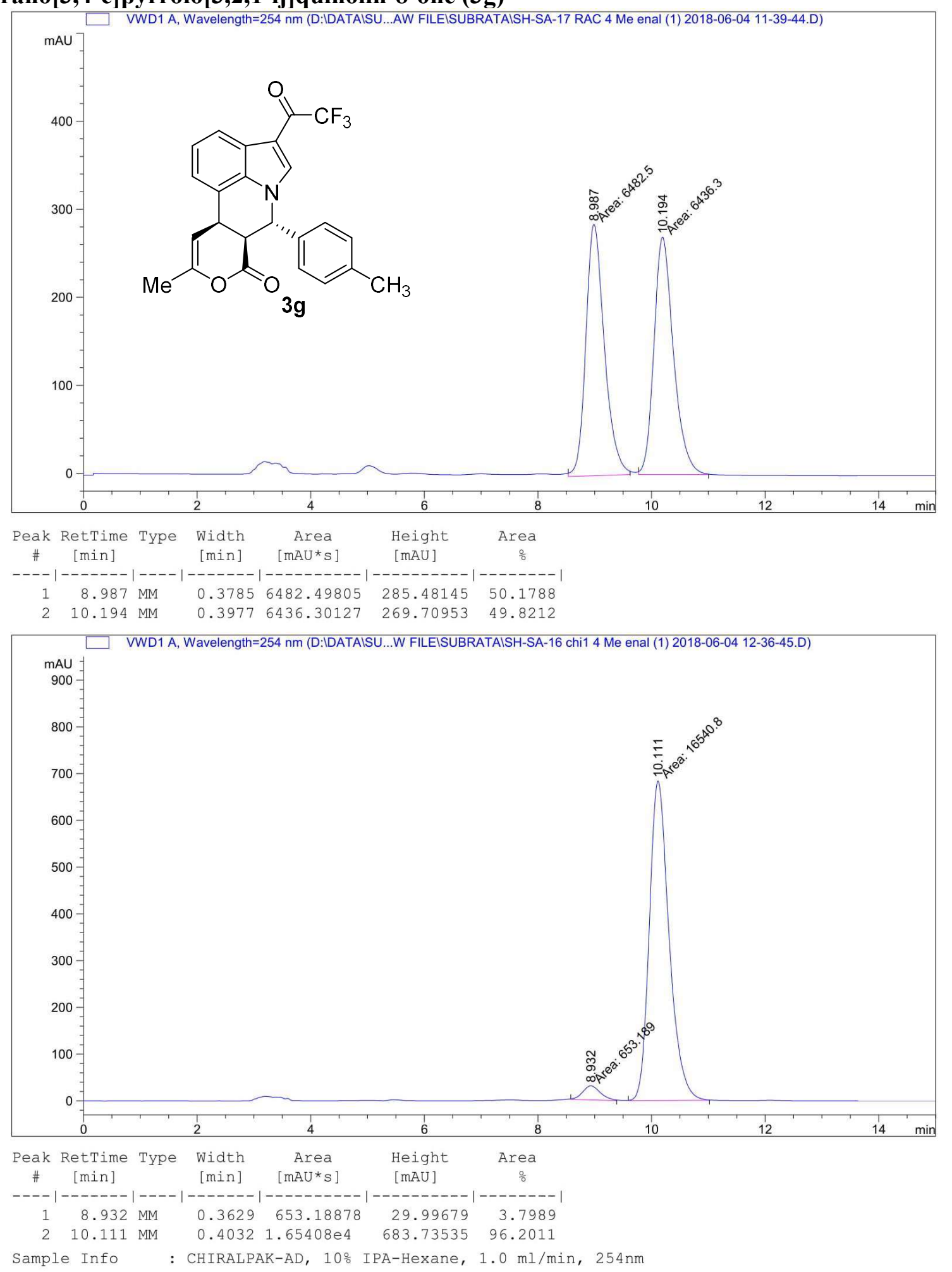

69 
(7S,7aS,11aR)-7-(4-Chlorophenyl)-10-methyl-4-(2,2,2-trifluoroacetyl)-7,7adihydropyrano[3,4-c]pyrrolo[3,2,1-ij]quinolin-8(11aH)-one (3h)

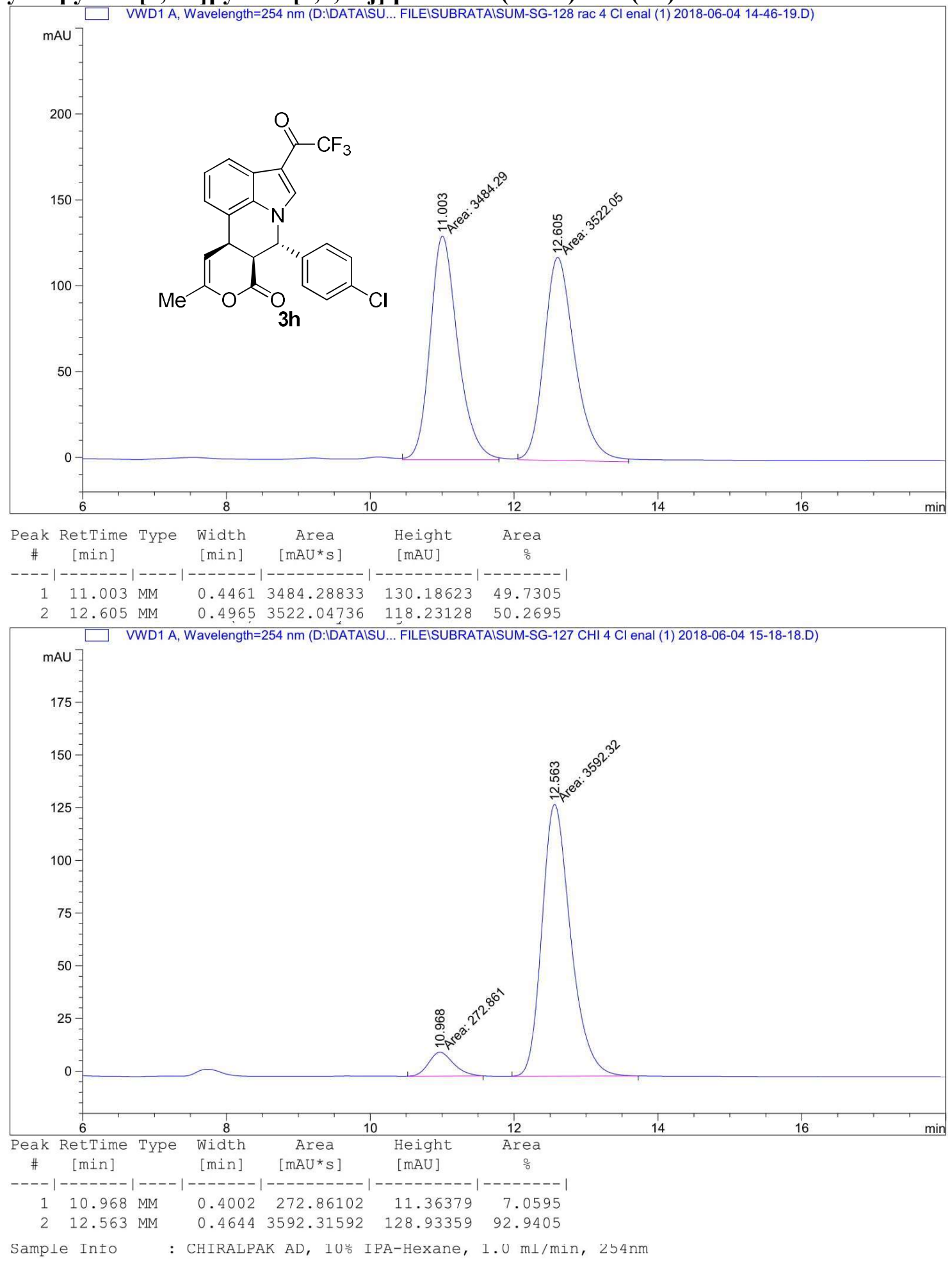

70 
(7S,7aS,11aR)-7-(4-Bromophenyl)-10-methyl-4-(2,2,2-trifluoroacetyl)-7a,11a-dihydro7H,8H-pyrano[3,4-c]pyrrolo[3,2,1-ij]quinolin-8-one (3i)
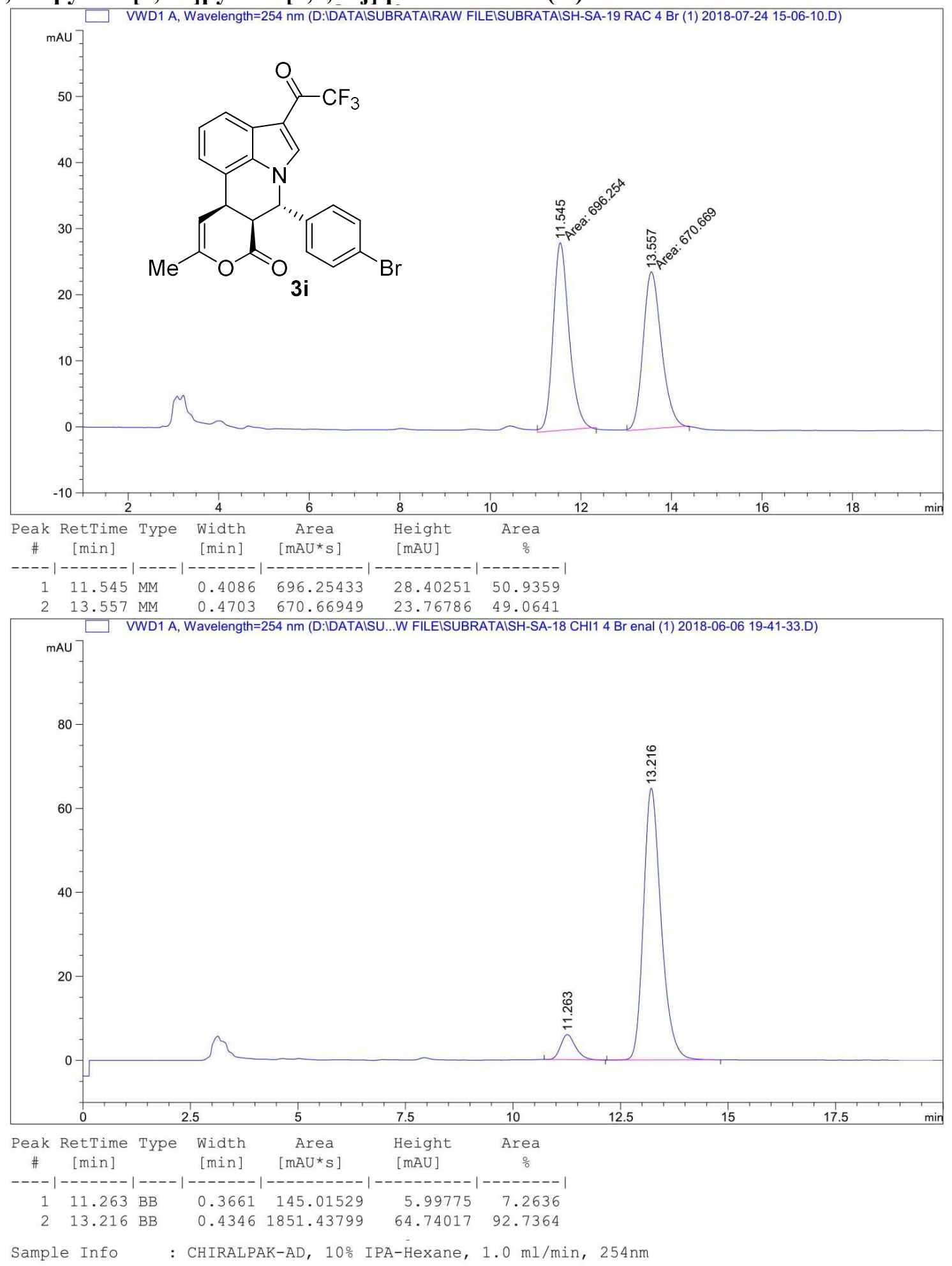
(7S,7aS,11a R)-10-Methyl-4-(2,2,2-trifluoroacetyl)-7-(4-(trifluoromethyl)phenyl)-7,7adihydropyrano[3,4-c]pyrrolo $[3,2,1$-ij]quinolin-8(11a $H)$-one $(3 \mathrm{j})$
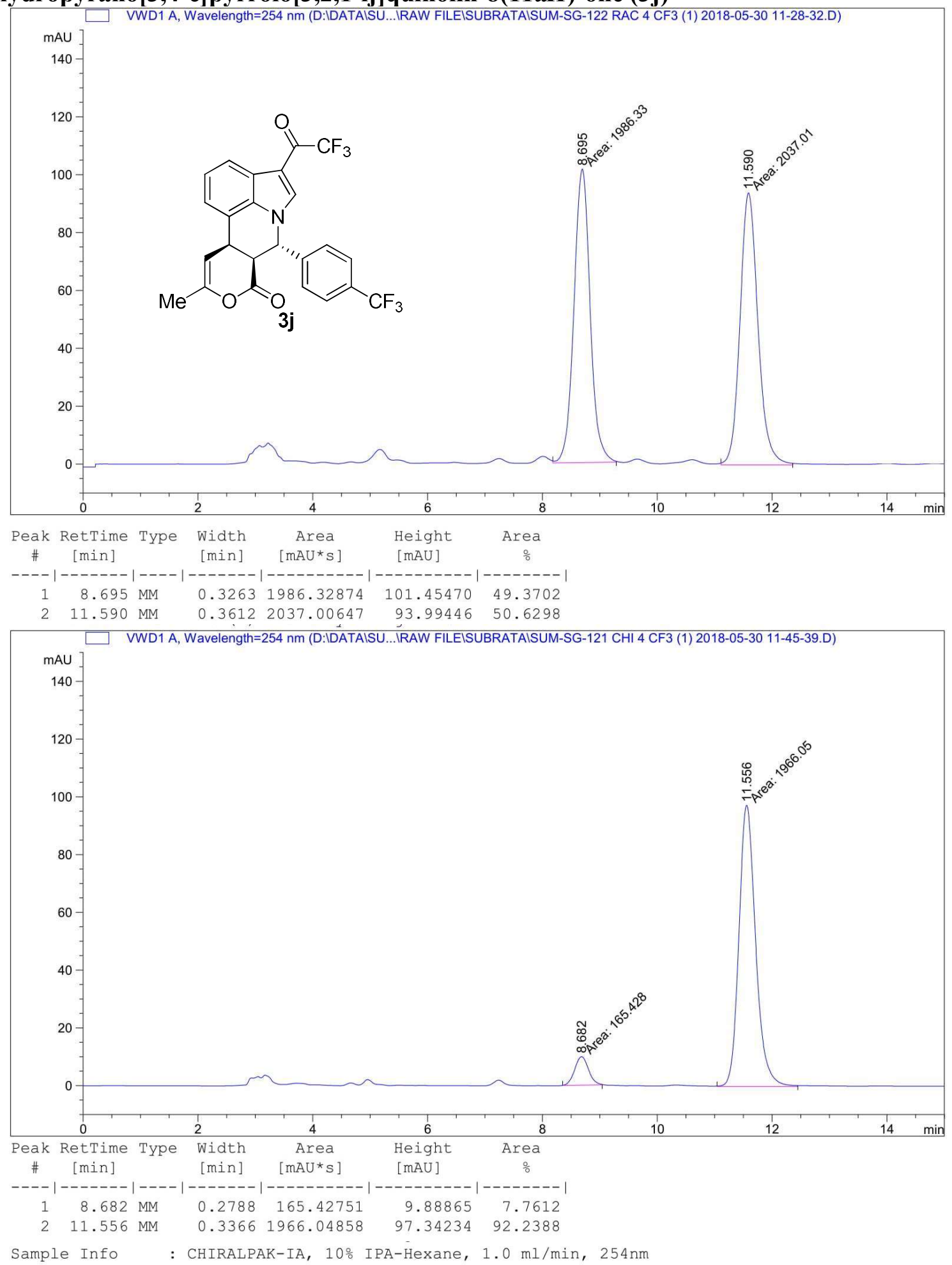
Methyl4-(7S,7aS,11aR)-10-methyl-8-oxo-4-(2,2,2-trifluoroacetyl)-7a,11a-dihydro-7H,8Hpyrano[3,4-c]pyrrolo[3,2,1-ij]quinolin-7-yl)benzoate (3k)
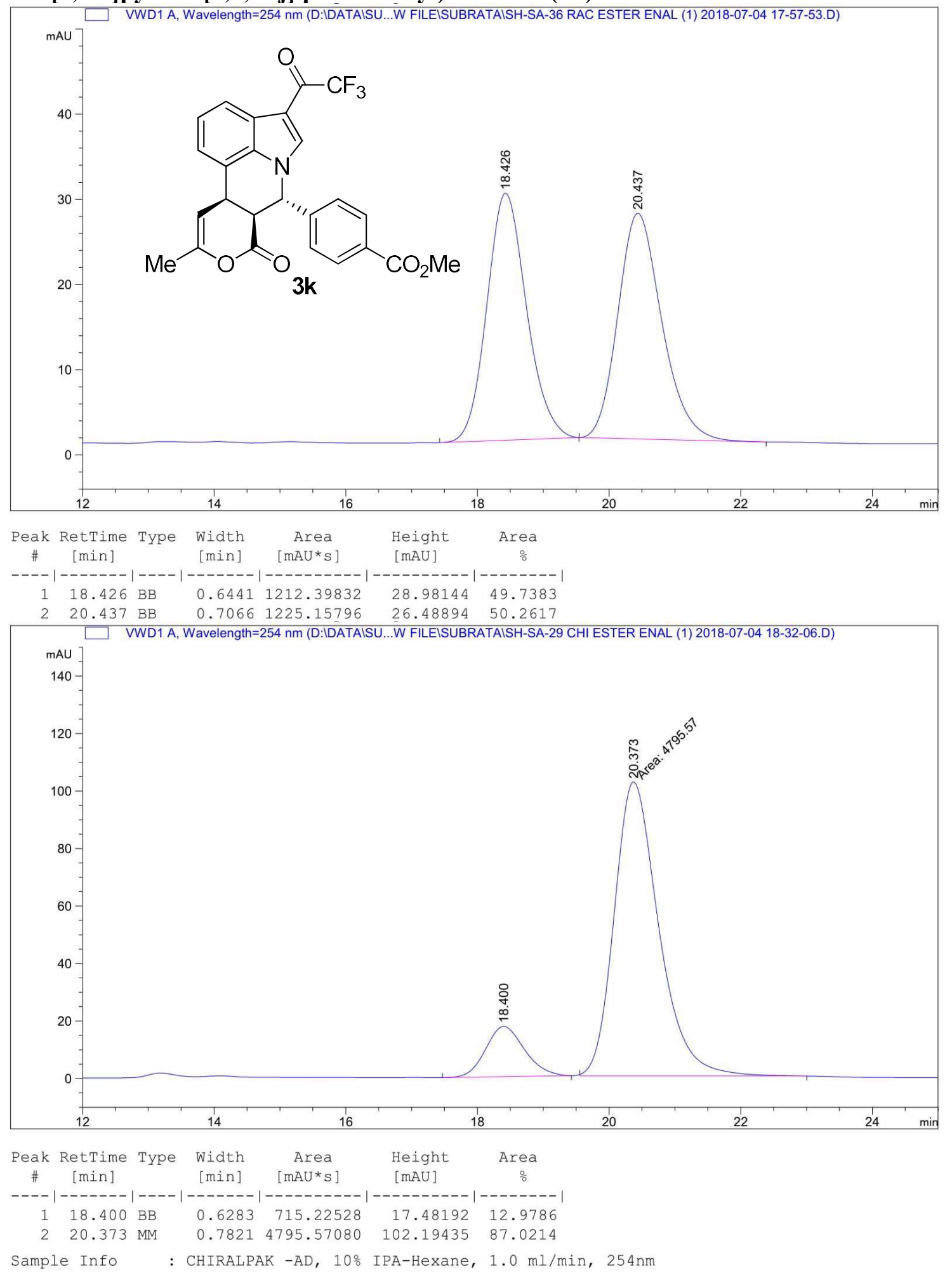

73 
(7S,7aS,11a R)-7-(3-Bromophenyl)-10-methyl-4-(2,2,2-trifluoroacetyl)-7,7adihydropyrano[3,4-c]pyrrolo[3,2,1-ij]quinolin-8(11aH)-one (3I)

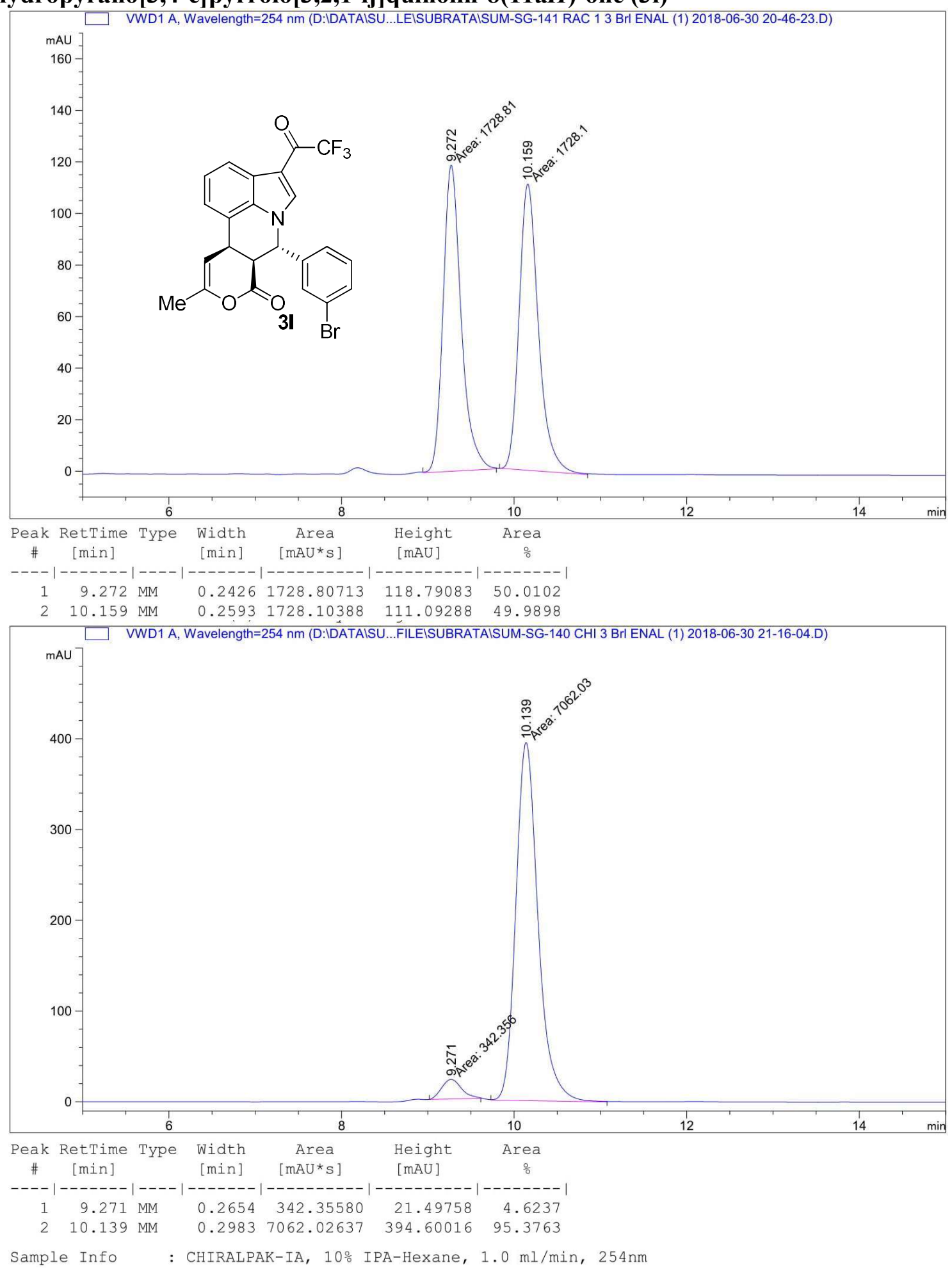

74 
(7S,7aS,11aR)-7-(2-Methoxyphenyl)-10-methyl-4-(2,2,2-trifluoroacetyl)-7a,11a-dihydro7H,8H-pyrano[3,4-c]pyrrolo[3,2,1-ij]quinolin-8-one (3m)
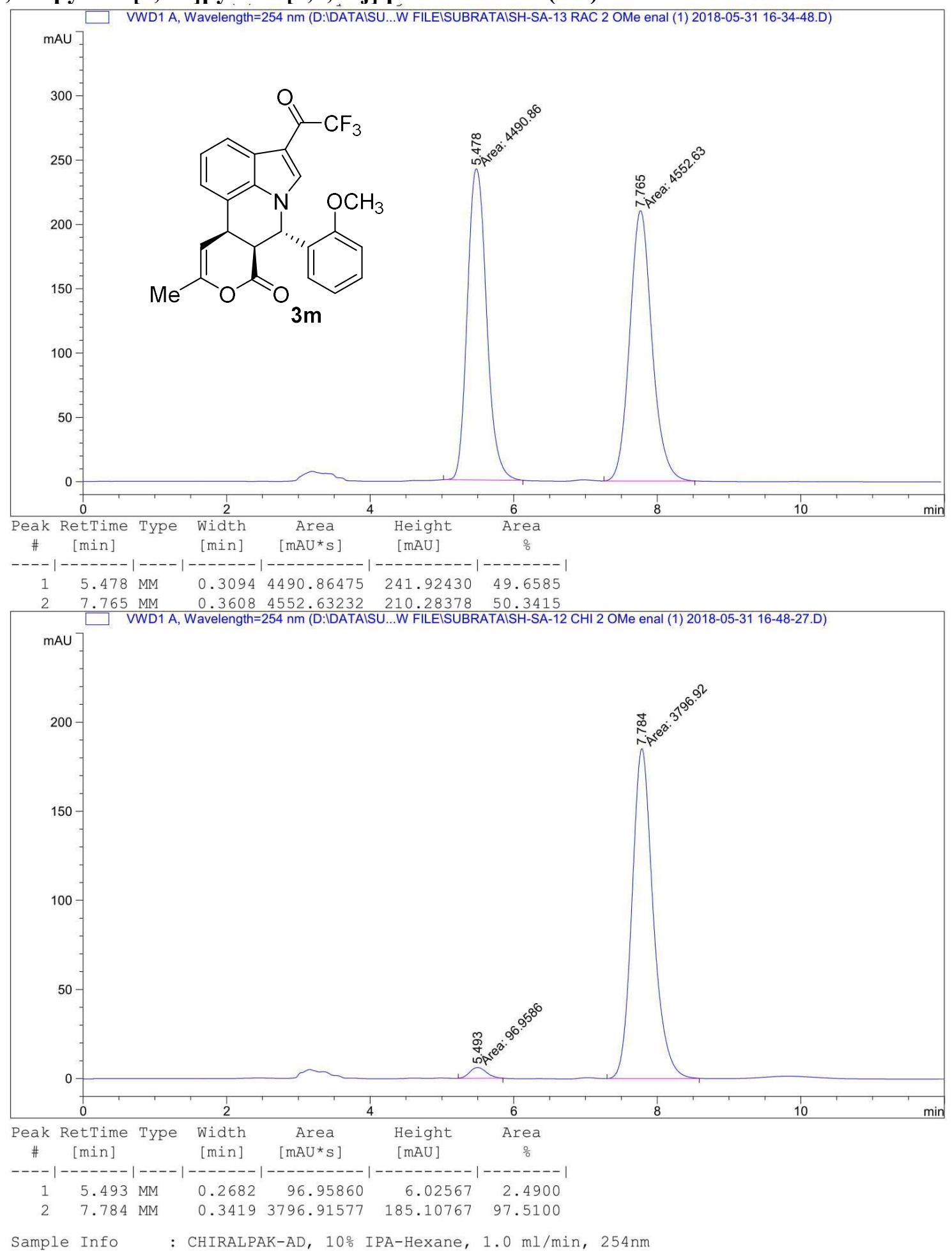
(7S,7aS,11a $R$ )-10-Methyl-7-(o-tolyl)-4-(2,2,2-trifluoroacetyl)-7,7a-dihydropyrano[3,4clpyrrolo[3,2,1-ij]quinolin-8(11aH)-one (3n)
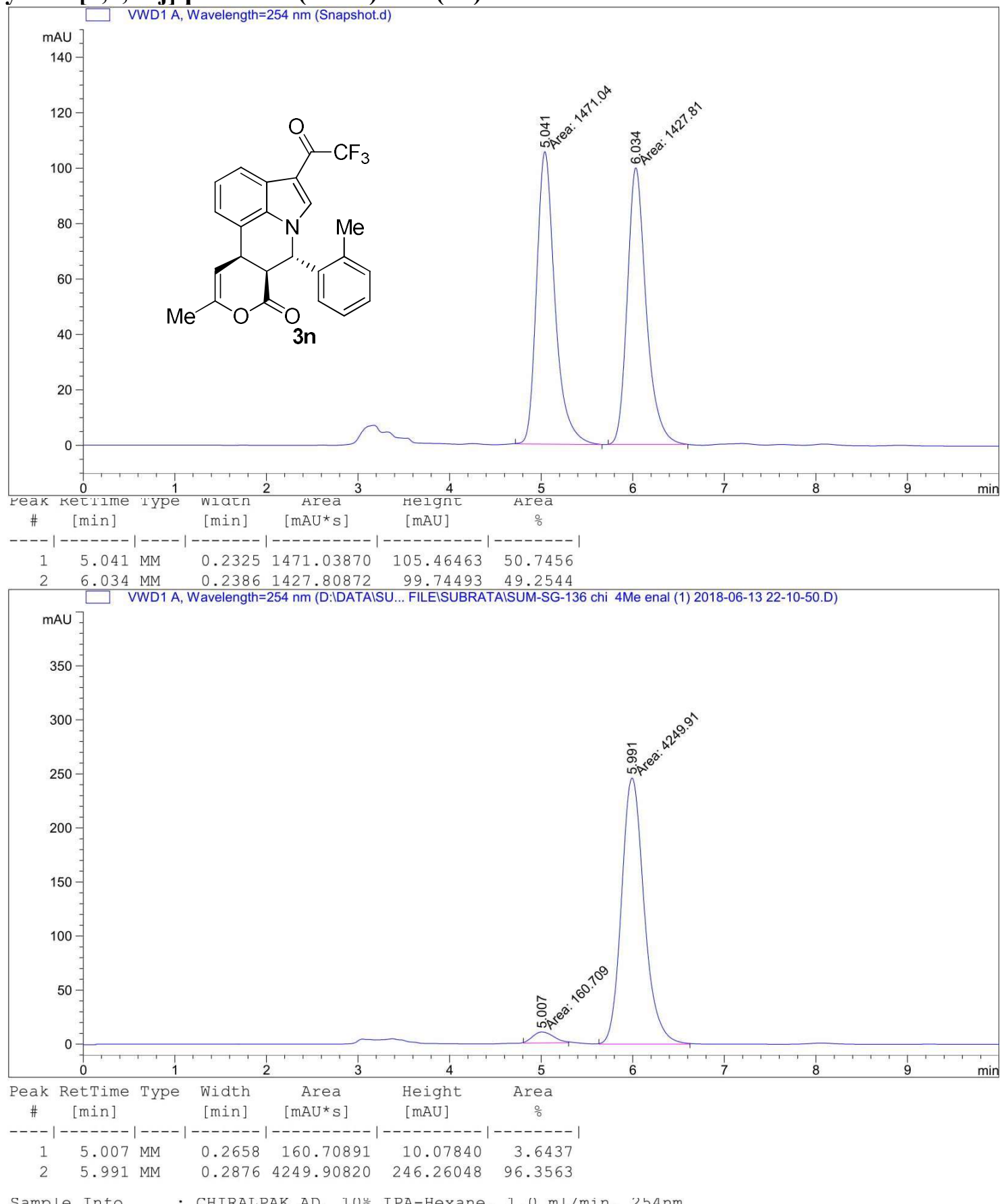

Sample Into : CHIRALPAK AD, $10 \%$ IPA-Hexane, $1.0 \mathrm{~m} \perp / \mathrm{min}, 254 \mathrm{~nm}$ 
(7S,7aS,11a $R$ )-7-(2-Fluorophenyl)-10-methyl-4-(2,2,2-trifluoroacetyl)-7,7adihydropyrano[3,4-c]pyrrolo[3,2,1-ij]quinolin-8(11aH)-one (3o)
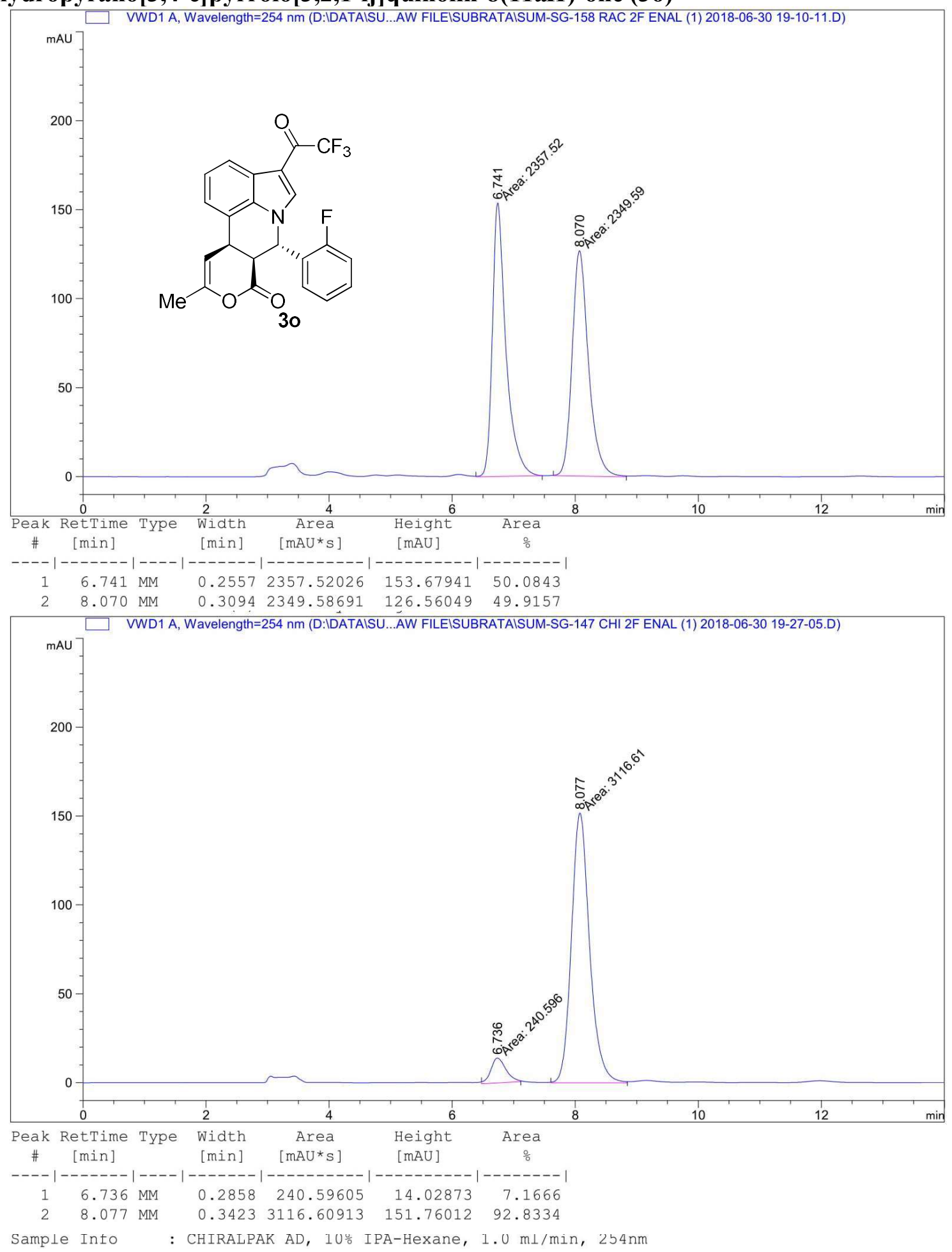
(7S,7aS,11a R)-7-(2-Chlorophenyl)-10-methyl-4-(2,2,2-trifluoroacetyl)-7,7adihydropyrano[3,4-c]pyrrolo[3,2,1-ij]quinolin-8(11aH)-one (3p)
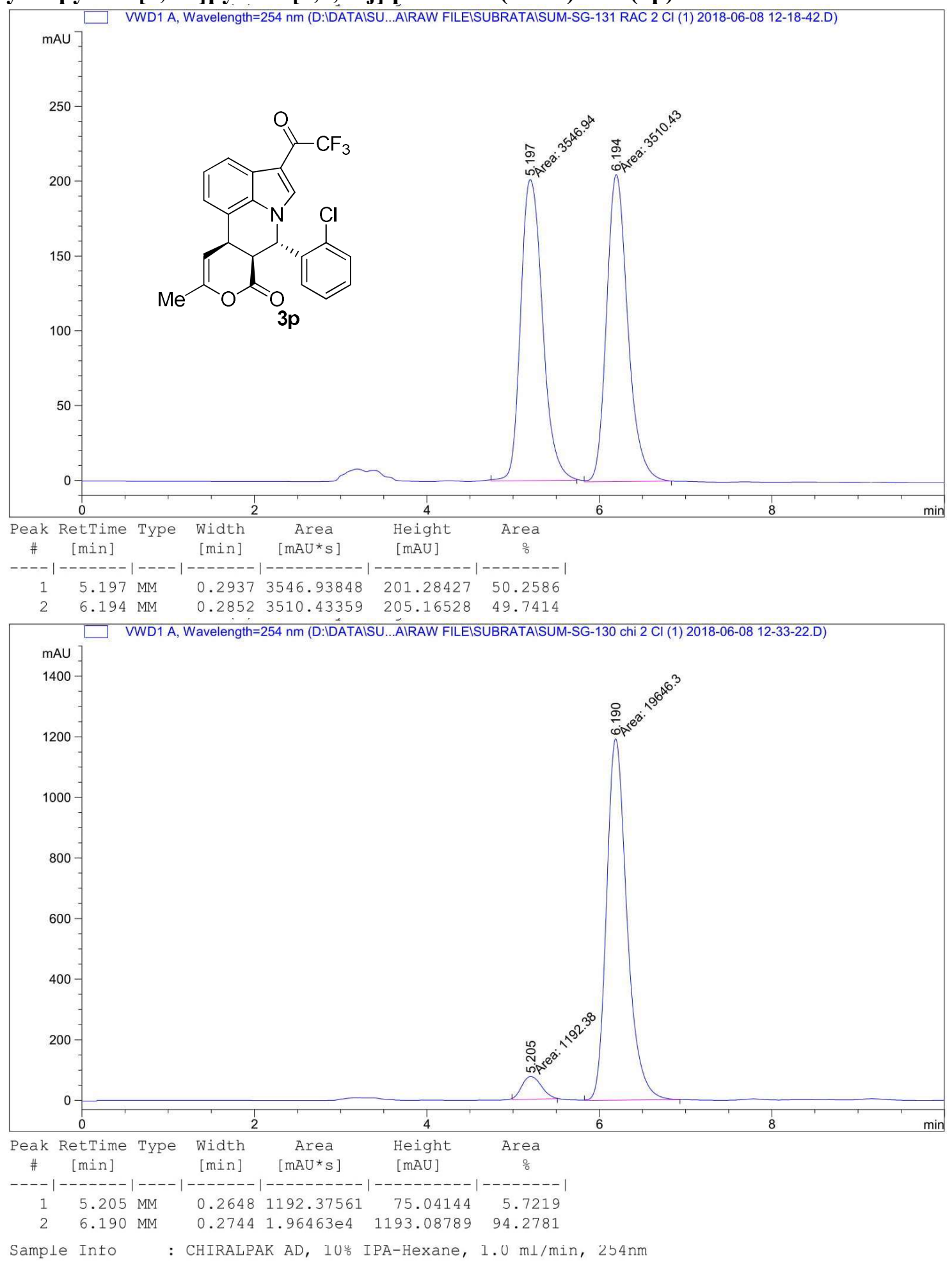
(7S,7aS,11aR)-7-(3,4-Dichlorophenyl)-10-methyl-4-(2,2,2-trifluoroacetyl)-7,7adihydropyrano[3,4-c]pyrrolo[3,2,1-ij]quinolin-8(11aH)-one (3q)
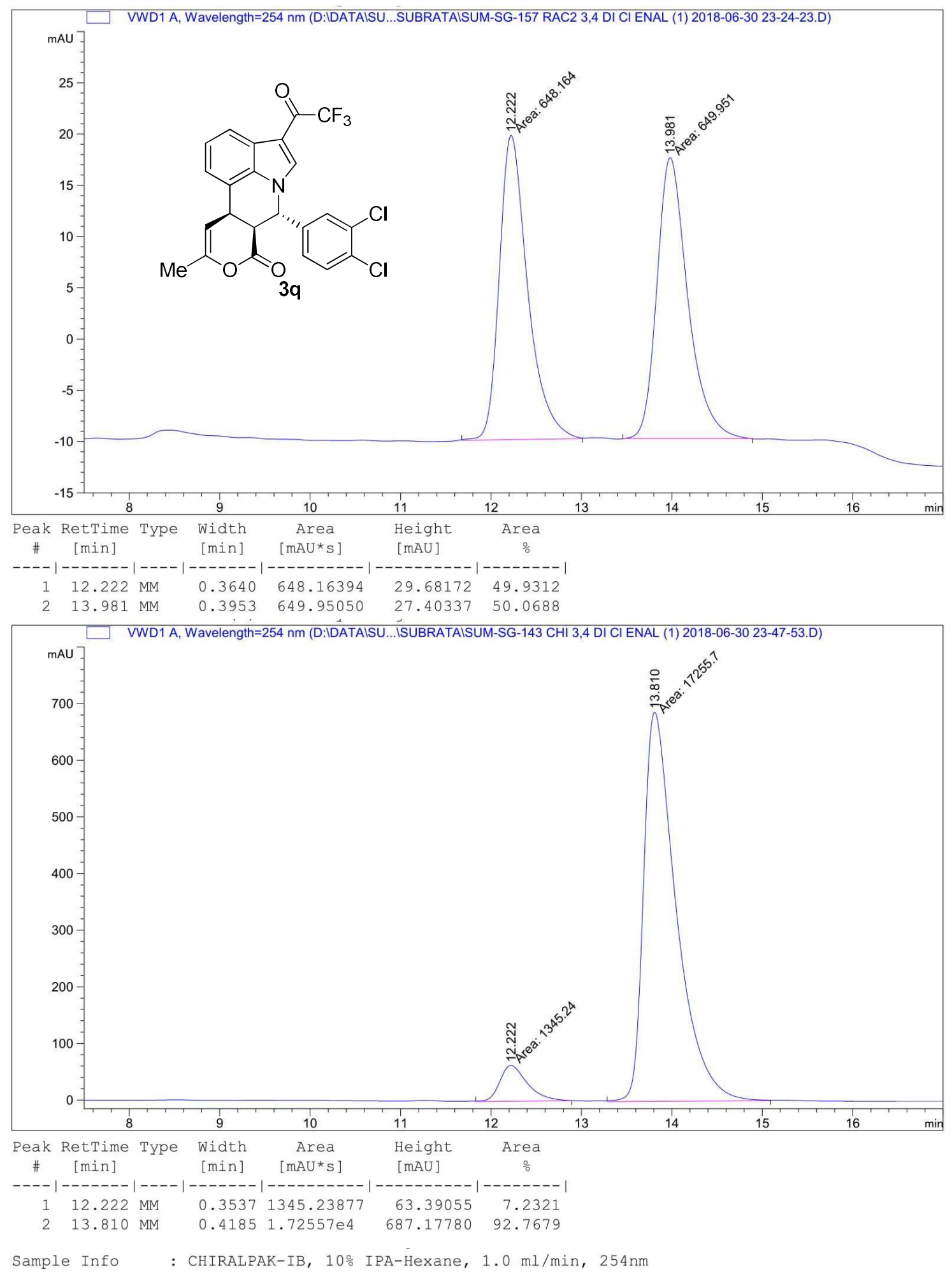
(7S,7aS,11aR)-7-(Benzo[d][1,3]dioxol-5-yl)-10-methyl-4-(2,2,2-trifluoroacetyl)-7,7adihydropyrano[3,4-c]pyrrolo[3,2,1-ij]quinolin-8(11a $H)$-one (3r)
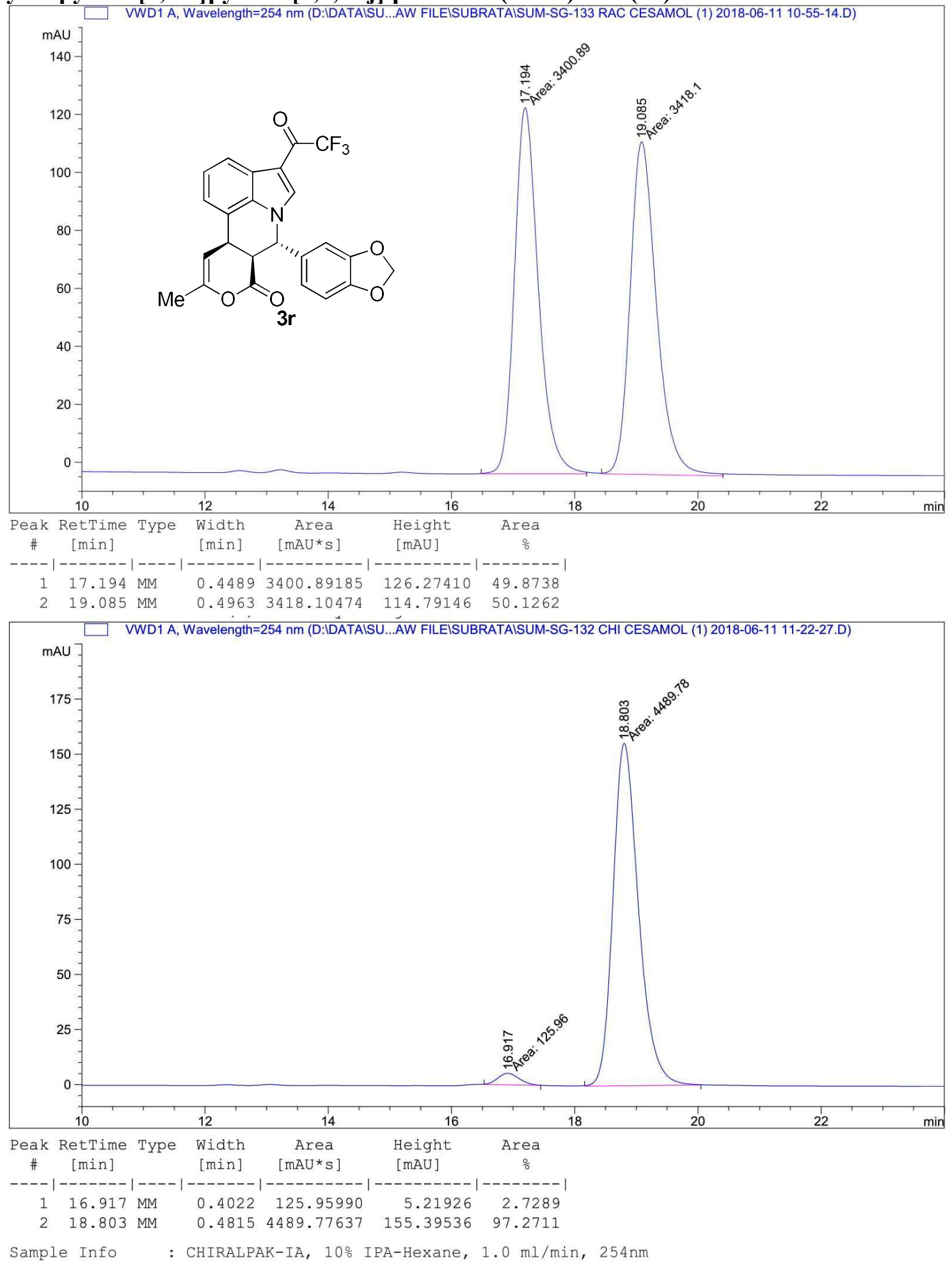
(7S,7aS,11aR)-10-Methyl-7-(naphthalen-2-yl)-4-(2,2,2-trifluoroacetyl)-7a,11a-dihydro7H,8H-pyrano[3,4-c]pyrrolo[3,2,1-ij]quinolin-8-one (3s)

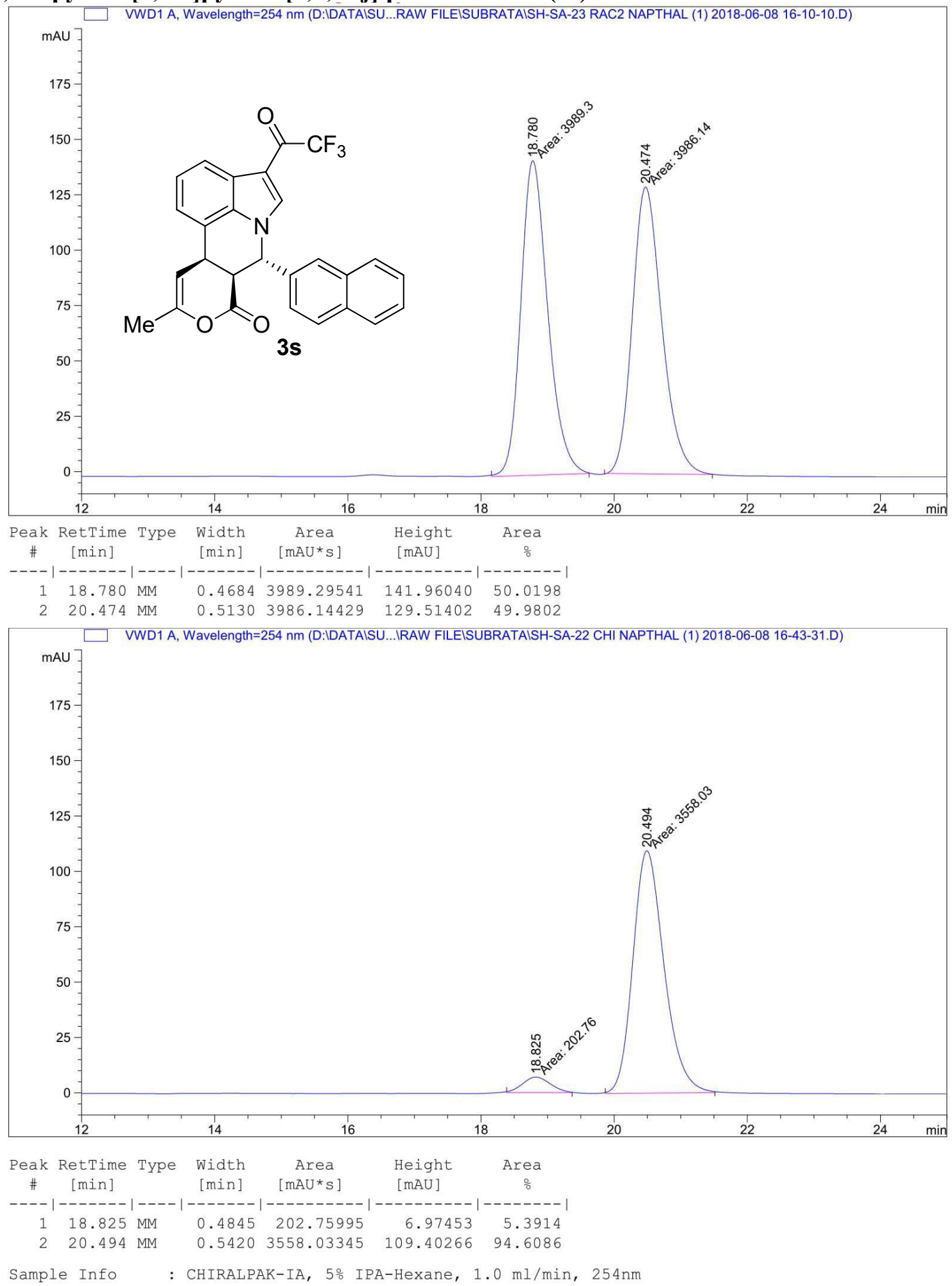

81 
(7S,7aS,11aR)-7-(Furan-2-yl)-10-methyl-4-(2,2,2-trifluoroacetyl)-7,7a-dihydropyrano[3,4c]pyrrolo[3,2,1-ij]quinolin-8(11aH)-one (3t)
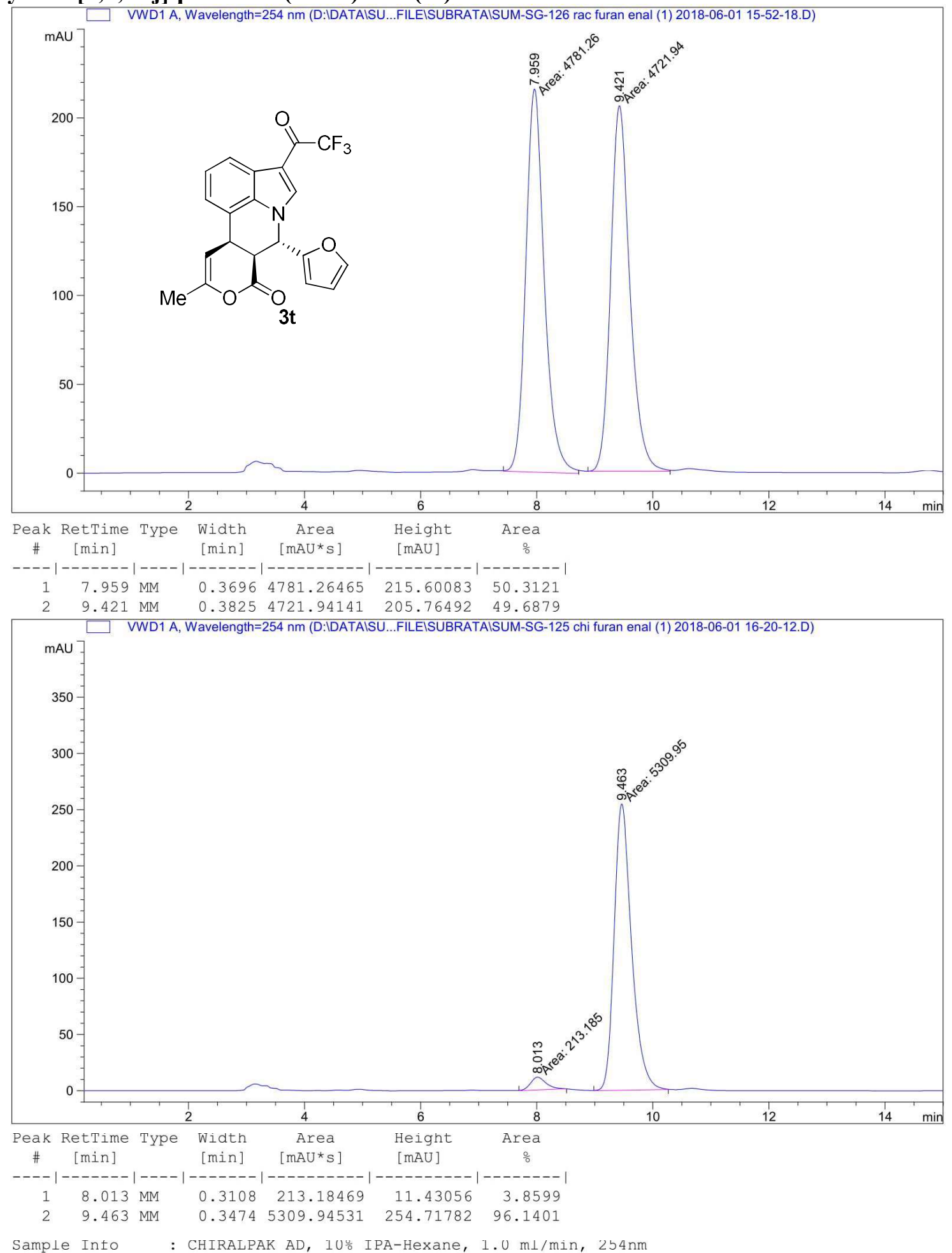
(7S,7aS,11aR)-10-Methyl-7-(thiophen-2-yl)-4-(2,2,2-trifluoroacetyl)-7,7adihydropyrano[3,4-c]pyrrolo[3,2,1-ij]quinolin-8(11aH)-one (3u)
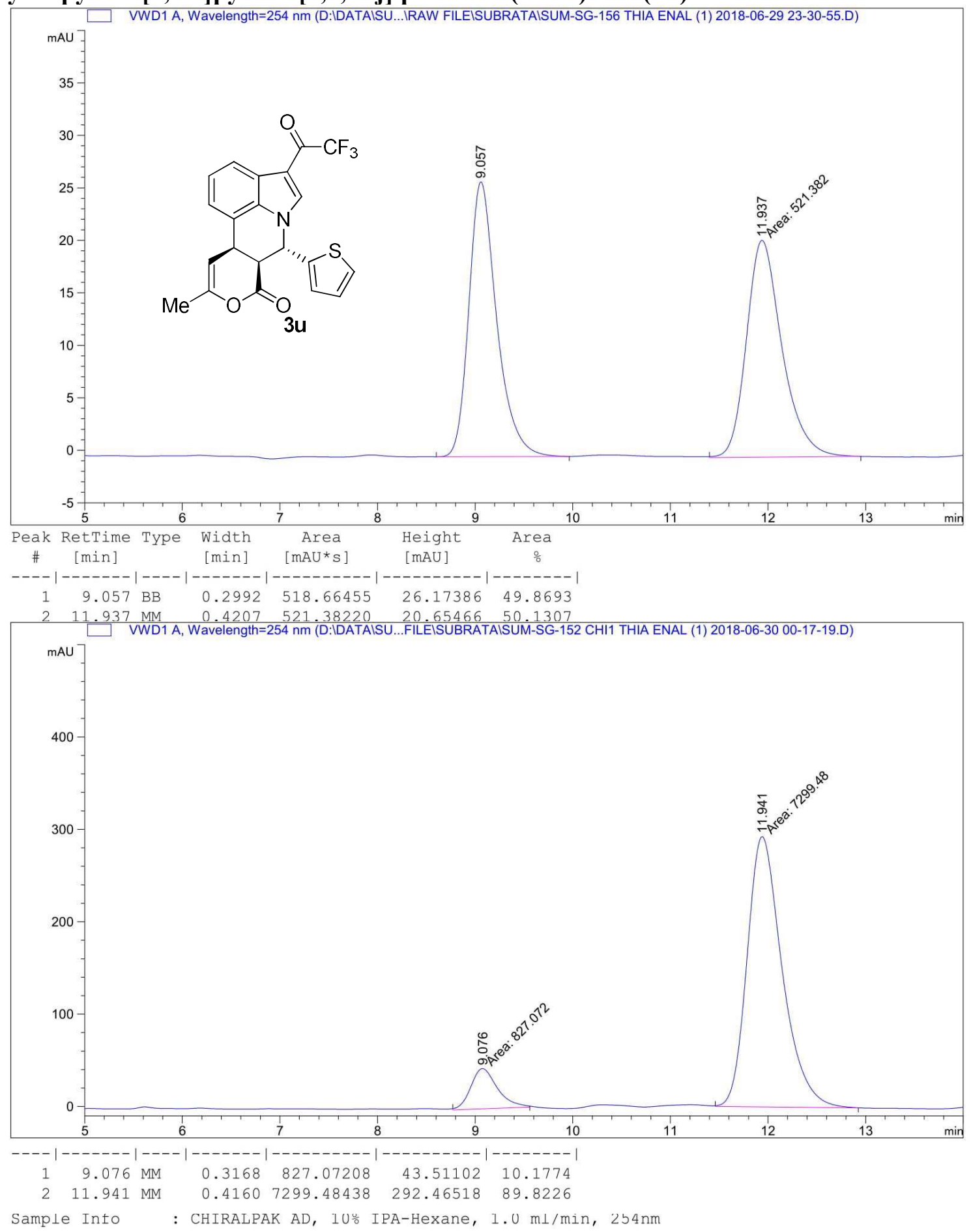
(7R,7aS,11aR)-10-Methyl-7-((E)-styryl)-4-(2,2,2-trifluoroacetyl)-7a,11a-dihydro-7H,8Hpyrano[3,4-c]pyrrolo[3,2,1-ij]quinolin-8-one (3v)

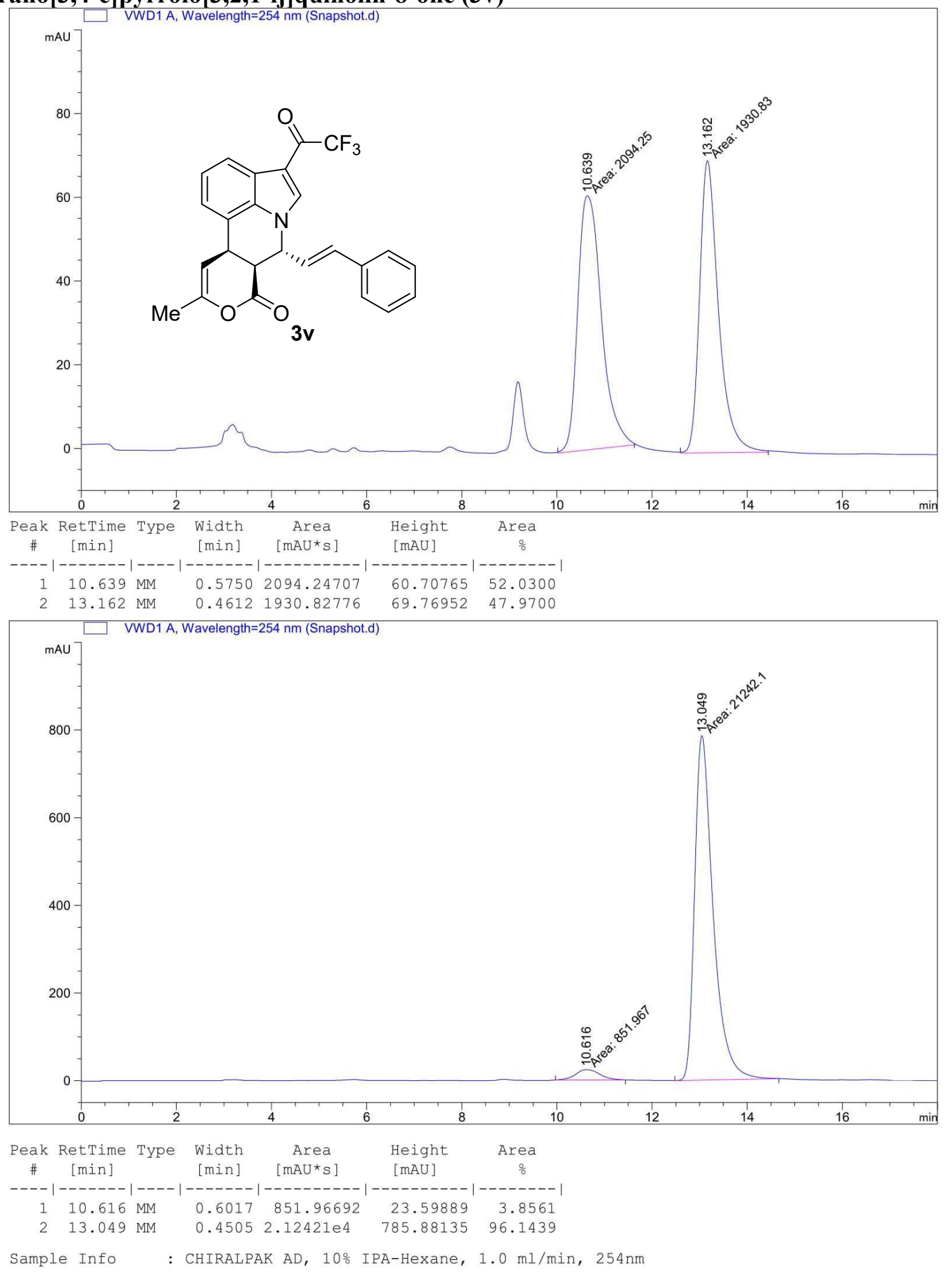

84 
(7S,7aS,11aR)-10-Cyclopropyl-7-phenyl-4-(2,2,2-trifluoroacetyl)-7,7a-dihydropyrano[3,4c]pyrrolo[3,2,1-ij]quinolin-8(11aH)-one (3w)
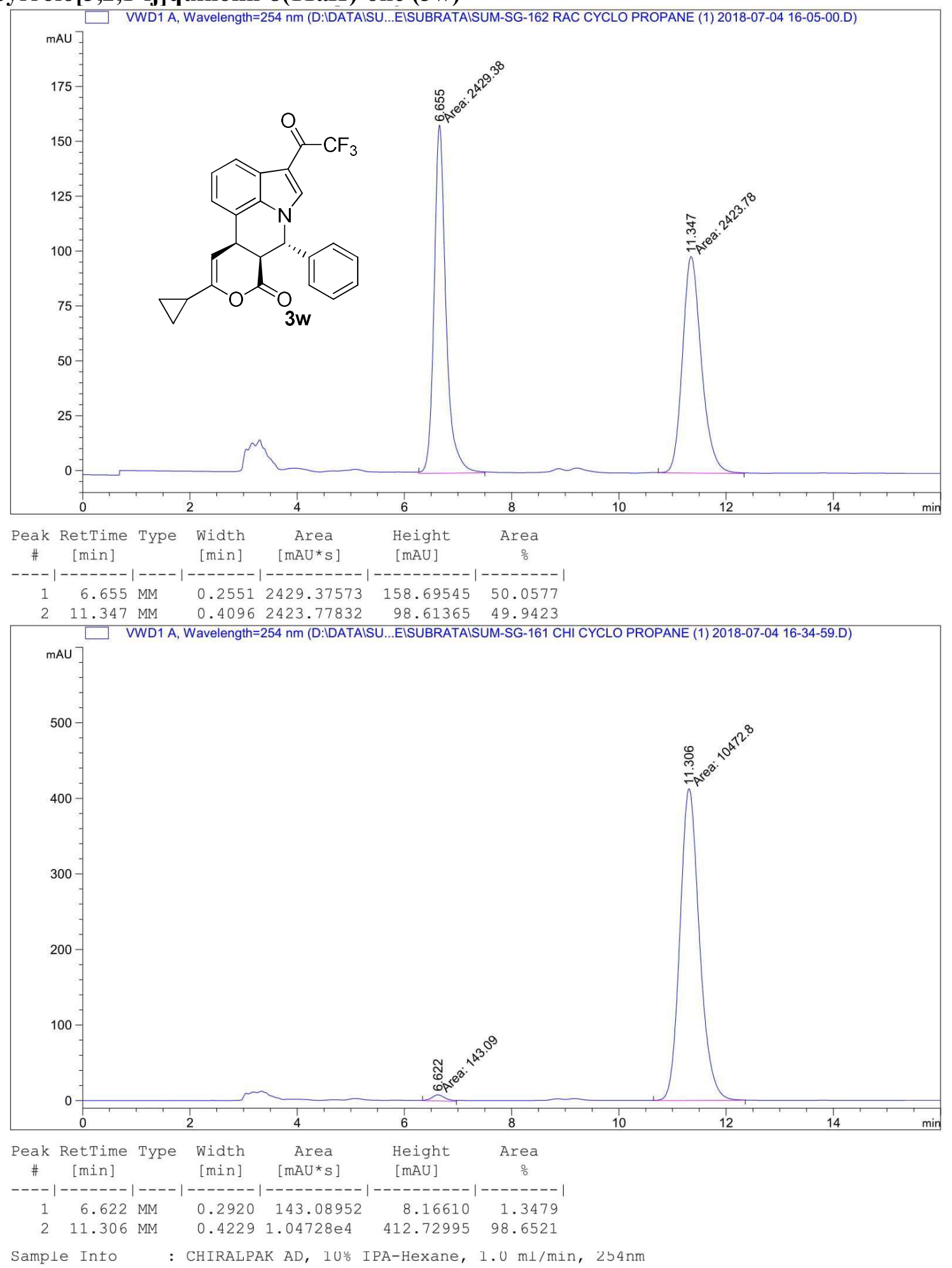
(7S,7aS,11a R)-10-Isobutyl-7-phenyl-4-(2,2,2-trifluoroacetyl)-7,7a-dihydropyrano[3,4c]pyrrolo[3,2,1-ij]quinolin-8(11aH)-one (3x)
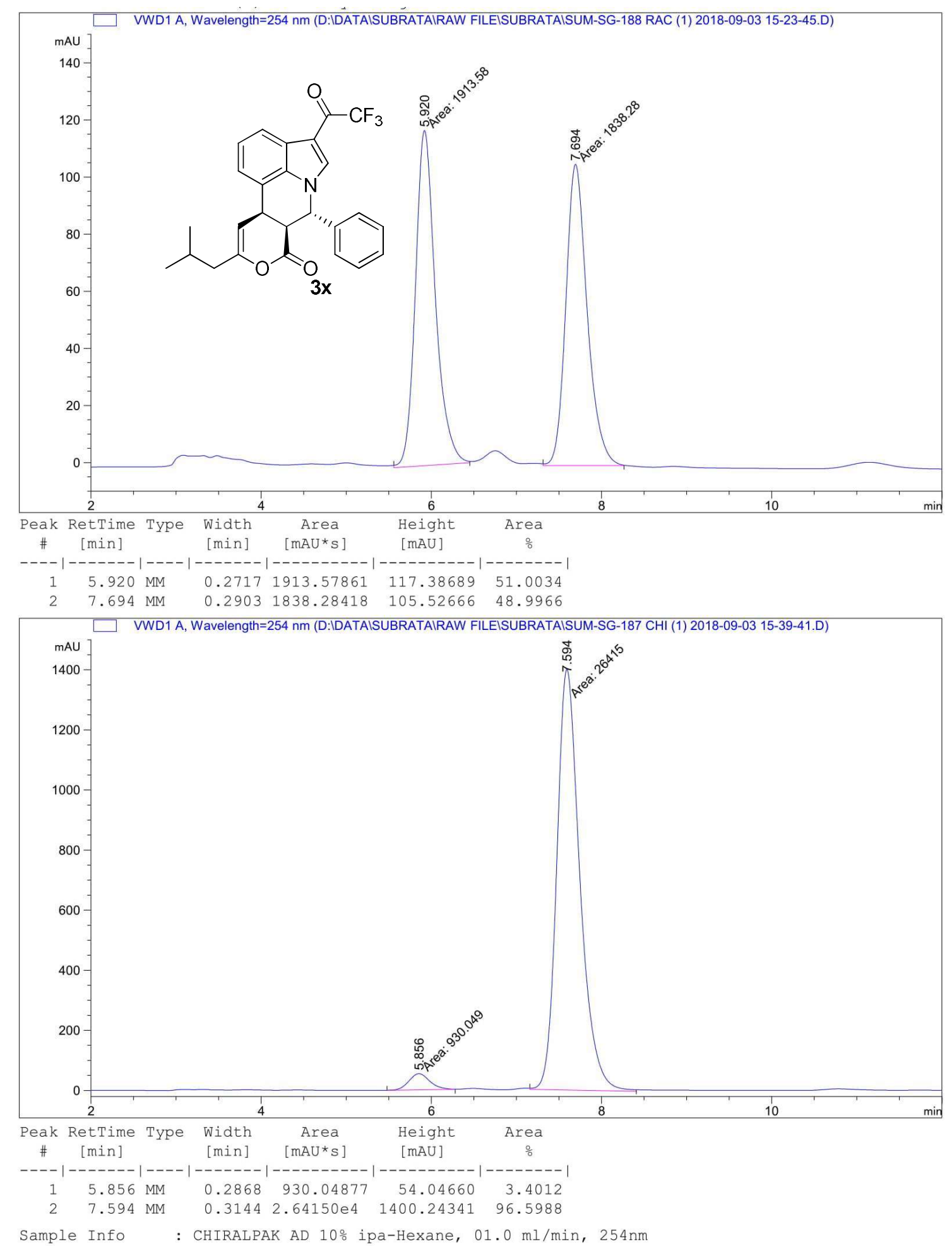
(7S,7aS,11a $R$ )-2-Bromo-10-methyl-7-phenyl-4-(2,2,2-trifluoroacetyl)-7,7adihydropyrano[3,4-c]pyrrolo[3,2,1-ij]quinolin-8(11a $H)$-one (3y)
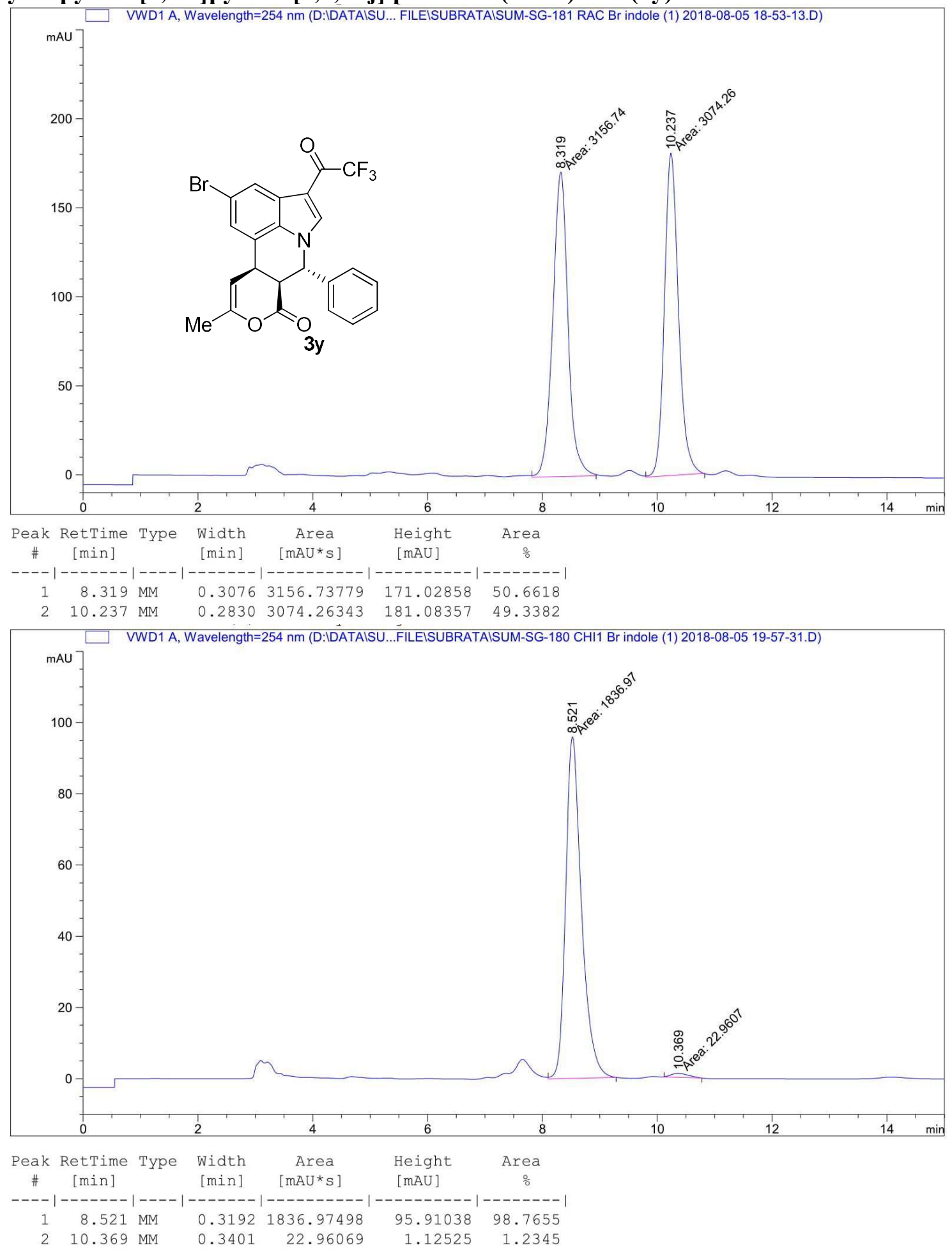

Sample Info : CHIRALPAK IA $10 \%$ ipa-Hexane, $1.0 \mathrm{ml} / \mathrm{min}, 254 \mathrm{~nm}$ 
$(4 S, 5 S, 6 R)$-Methyl

6-(2-oxopropyl)-4-phenyl-1-(2,2,2-trifluoroacetyl)-5,6-dihydro-4H-

\section{pyrrolo[3,2,1-ij]quinoline-5-carboxylate (6d)}

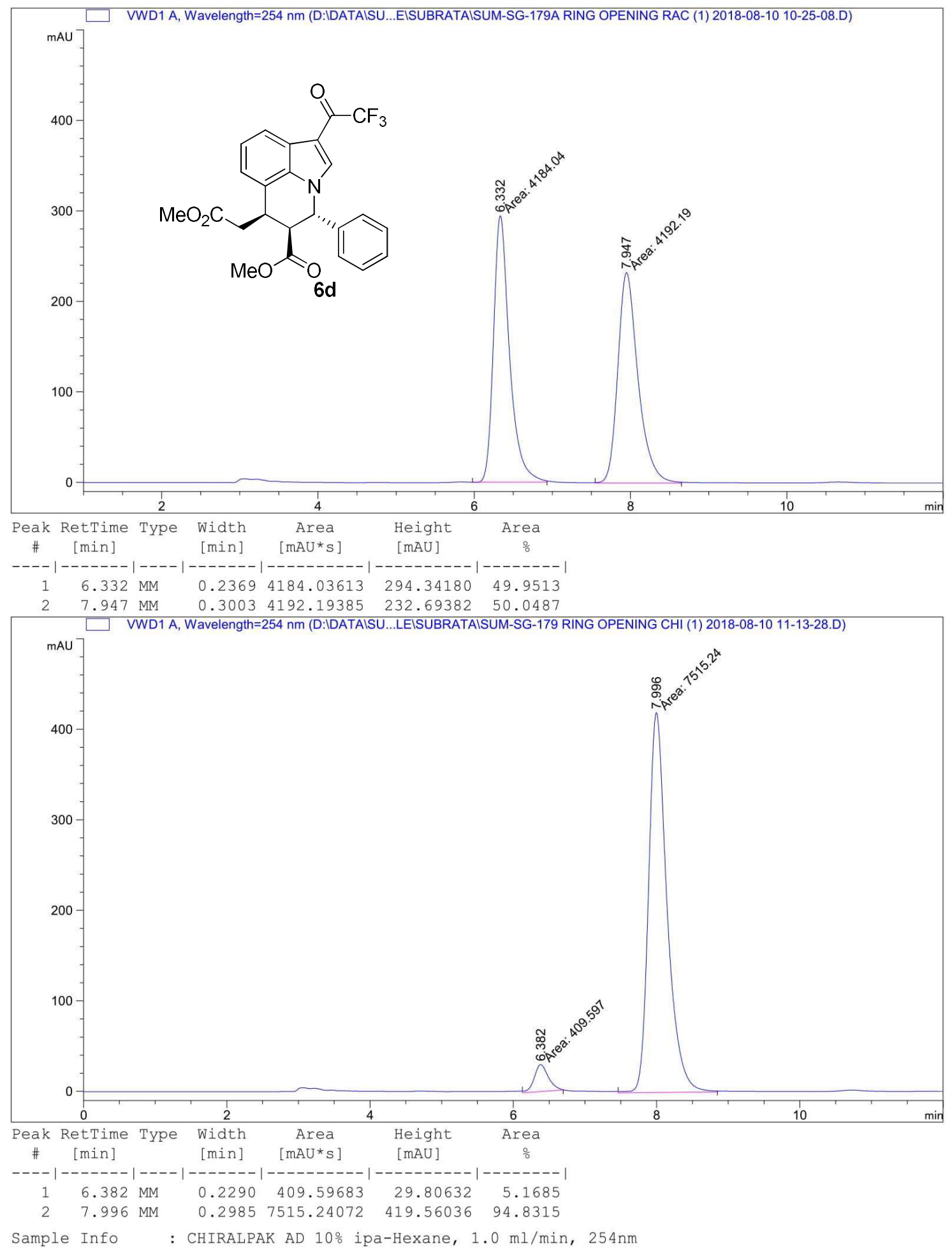


(4S,5S,6R)-N-Benzyl-6-(2-oxopropyl)-4-phenyl-1-(2,2,2-trifluoroacetyl)-5,6-dihydro-4Hpyrrolo[3,2,1-ij]quinoline-5-carboxamide (7d)
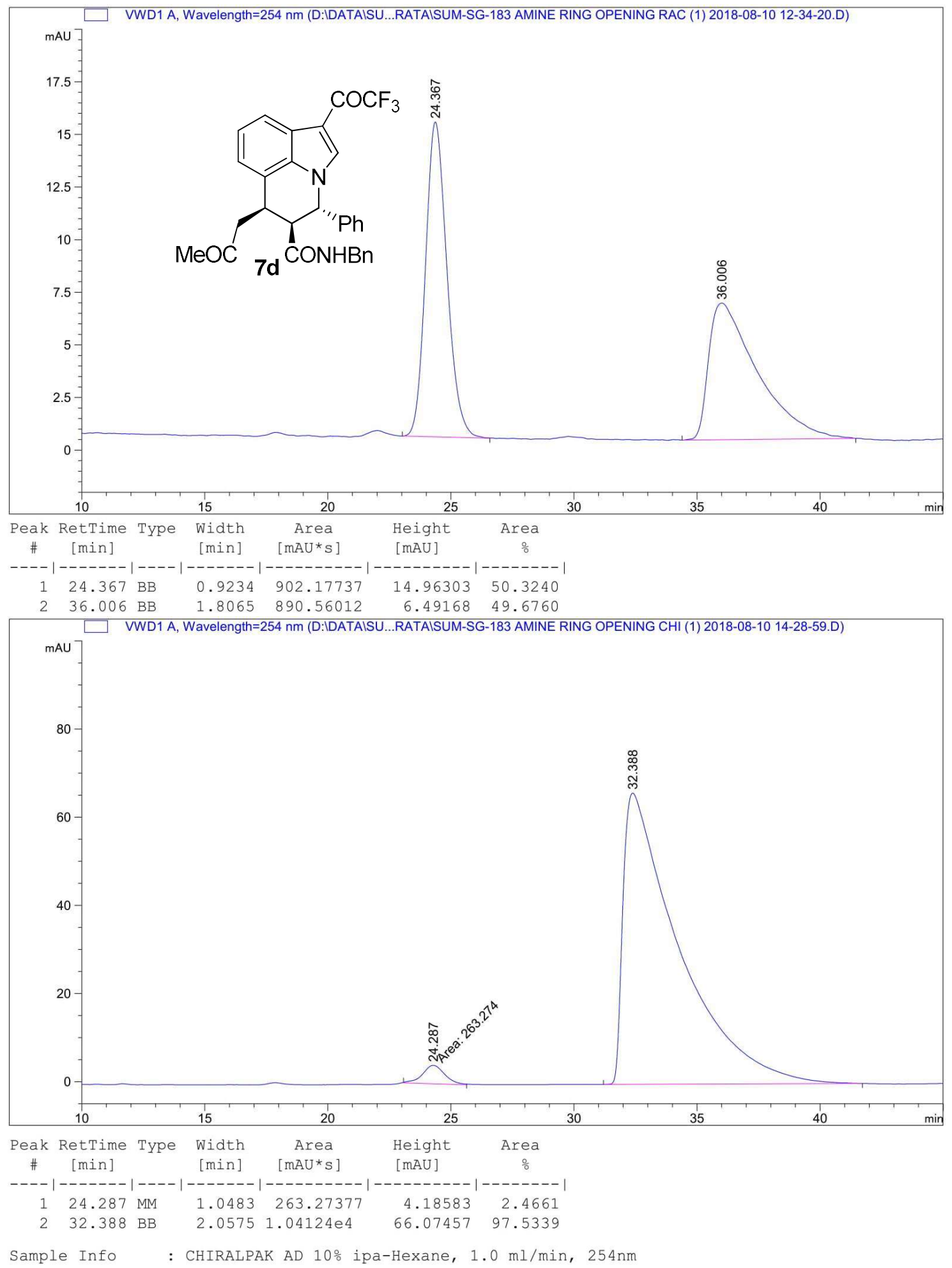
(E)-Methyl 3-((7S,7aS,11aR)-10-methyl-8-oxo-7-phenyl-4-(2,2,2-trifluoroacetyl)-7,7a,8,11atetrahydropyrano[3,4-c]pyrrolo[3,2,1-ij]quinolin-3-yl)acrylate (8d)
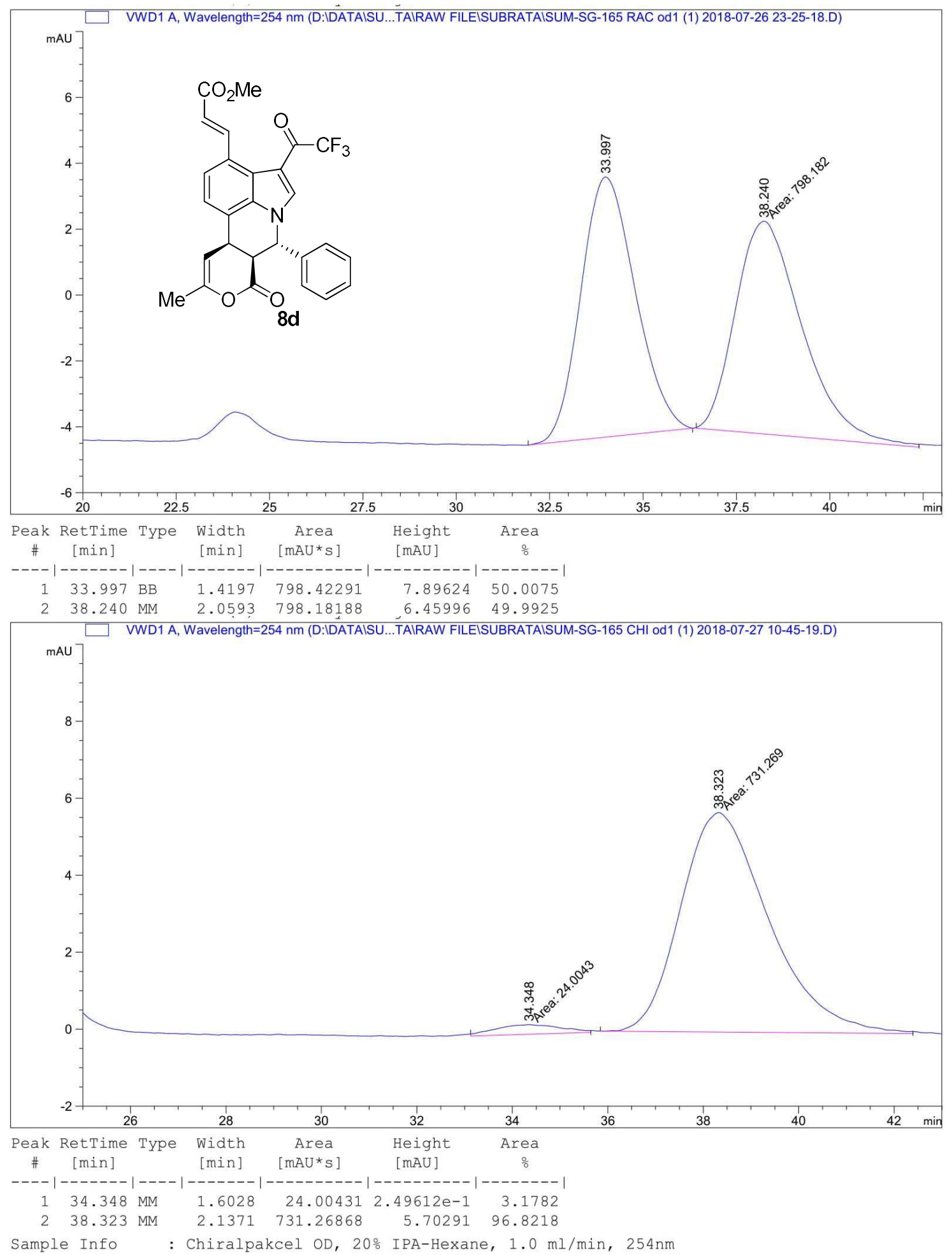
1-Benzyl-3-((7S,7aS,11aR)-10-methyl-8-oxo-7-phenyl-4-(2,2,2-trifluoroacetyl)7,7a,8,11atetrahydropyrano[3,4-c]pyrrolo[3,2,1-ij]quinolin-3-yl)pyrrolidine-2,5-dione (9d)

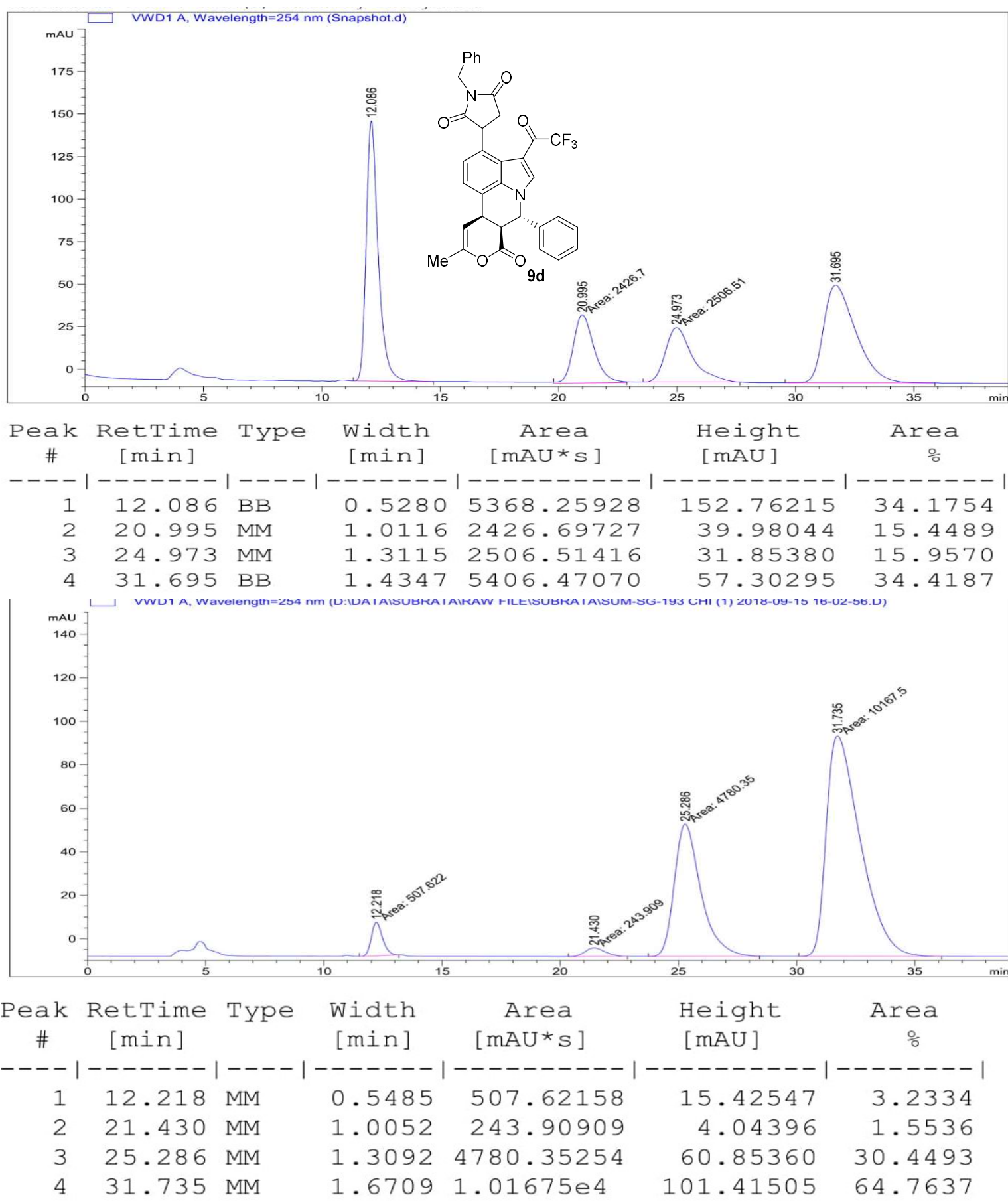

Sample Info : CHIRALPAK AD 20\% ipa-Hexane, $1.0 \mathrm{ml} / \mathrm{min}, 254 \mathrm{~nm}$ 